\title{
Plumbagin elicits differential proteomic responses mainly involving cell cycle, apoptosis, autophagy, and epithelial-to-mesenchymal transition pathways in human prostate cancer PC-3 and DUI45 cells
}

\author{
This article was published in the following Dove Press journal: \\ Drug Design, Development and Therapy \\ 7 January 2015 \\ Number of times this article has been viewed
}

\author{
Jia-Xuan Qiu' ${ }^{1,2}$ \\ Zhi-Wei Zhou ${ }^{3,4}$ \\ Zhi-Xu He ${ }^{4}$ \\ Ruan Jin Zhao ${ }^{5}$ \\ Xueji Zhang ${ }^{6}$ \\ Lun Yang ${ }^{7}$ \\ Shu-Feng Zhou ${ }^{3,4}$ \\ Zong-Fu Mao'
}

'School of Public Health, Wuhan University, Wuhan, Hubei, People's Republic of China;

${ }^{2}$ Department of Oral and Maxillofacial

Surgery, The First Affiliated Hospital

of Nanchang University, Nanchang, Jiangxi,

People's Republic of China; ${ }^{3}$ Department

of Pharmaceutical Sciences, College

of Pharmacy, University of South Florida,

Tampa, FL, USA; ${ }^{4}$ Guizhou Provincial Key

Laboratory for Regenerative Medicine,

Stem Cell and Tissue Engineering Research

Center and Sino-US Joint Laboratory

for Medical Sciences, Guiyang Medical

University, Guiyang, Guizhou, People's

Republic of China; ${ }^{5}$ Center for Traditional

Chinese Medicine, Sarasota, FL, USA;

${ }^{6}$ Research Center for Bioengineering and

Sensing Technology, University of Science and Technology Beijing, Beijing, People's

Republic of China; ${ }^{7}$ Bio-X Institutes,

Key Laboratory for the Genetics

of Development and Neuropsychiatric

Disorders (Ministry of Education), Shanghai

Jiao Tong University, Shanghai, People's

Republic of China

Correspondence: Zong-Fu Mao

School of Public Health, Wuhan University,

185 Donghu Road, Wuchang District, Wuhan

43007I, Hubei, People's Republic of China

Tel +86276875 9609

Email zfmao@I26.com

Shu-Feng Zhou

Department of Pharmaceutical Sciences,

College of Pharmacy, University of South

Florida, I290I Bruce B Downs Blvd., MDC 30,

Tampa, FL 33612, USA

Tel +l 8I3974 6276

Fax + I 8139059885

Email szhou@health.usf.edu
Abstract: Plumbagin (PLB) has exhibited a potent anticancer effect in preclinical studies, but the molecular interactome remains elusive. This study aimed to compare the quantitative proteomic responses to PLB treatment in human prostate cancer PC-3 and DU145 cells using the approach of stable-isotope labeling by amino acids in cell culture (SILAC). The data were finally validated using Western blot assay. First, the bioinformatic analysis predicted that PLB could interact with 78 proteins that were involved in cell proliferation and apoptosis, immunity, and signal transduction. Our quantitative proteomic study using SILAC revealed that there were at least 1,225 and 267 proteins interacting with PLB and there were 341 and 107 signaling pathways and cellular functions potentially regulated by PLB in PC-3 and DU145 cells, respectively. These proteins and pathways played a critical role in the regulation of cell cycle, apoptosis, autophagy, epithelial to mesenchymal transition (EMT), and reactive oxygen species generation. The proteomic study showed substantial differences in response to PLB treatment between PC-3 and DU145 cells. PLB treatment significantly modulated the expression of critical proteins that regulate cell cycle, apoptosis, and EMT signaling pathways in PC-3 cells but not in DU145 cells. Consistently, our Western blotting analysis validated the bioinformatic and proteomic data and confirmed the modulating effects of PLB on important proteins that regulated cell cycle, apoptosis, autophagy, and EMT in PC-3 and DU145 cells. The data from the Western blot assay could not display significant differences between PC-3 and DU145 cells. These findings indicate that PLB elicits different proteomic responses in PC-3 and DU145 cells involving proteins and pathways that regulate cell cycle, apoptosis, autophagy, reactive oxygen species production, and antioxidation/oxidation homeostasis. This is the first systematic study with integrated computational, proteomic, and functional analyses revealing the networks of signaling pathways and differential proteomic responses to PLB treatment in prostate cancer cells. Quantitative proteomic analysis using SILAC represents an efficient and highly sensitive approach to identify the target networks of anticancer drugs like PLB, and the data may be used to discriminate the molecular and clinical subtypes, and to identify new therapeutic targets and biomarkers, for prostate cancer. Further studies are warranted to explore the potential of quantitative proteomic analysis in the identification of new targets and biomarkers for prostate cancer.

Keywords: EMT, proteomics, SILAC

\section{Introduction}

Prostate cancer is the second most common cancer in men worldwide, after lung cancer. ${ }^{1}$ There were over 903,500 new prostate cancer cases reported worldwide and 
an estimated 258,400 men died from this disease in $2008 .^{2}$ The incidence of prostate cancer varies significantly among different countries and ethnic groups. It is quite frequently diagnosed in North America and Europe but is rare in Asians. ${ }^{3-5}$ The age-standardized incidence rate of prostate cancer in the People's Republic of China was 4.3 per $10^{5}$, but it is 83.8 per $10^{5}$ in the US. ${ }^{3,4}$ In the US, 196,038 men were diagnosed with prostate cancer, and 28,560 American men died from this disease in $2010 .^{6,7}$ In the United Kingdom, 40,975 men were diagnosed with prostate cancer in 2010 , and 10,793 men died from this disease in 2011. ${ }^{8}$ Although the 10-year survival rate for early prostate cancer was over $98 \%$ in the US, many patients were diagnosed with locally advanced or metastatic forms of prostate cancer in clinic. ${ }^{9,10}$ This will substantially and negatively affect the therapeutic outcomes. Current prostate cancer therapy includes surgery, radiation, hormone therapy, and chemotherapy. ${ }^{11}$ Androgen-deprivation therapy with antiandrogens remains the main treatment for later-stage prostate cancer, and it can effectively suppress prostate cancer growth during the first 12-24 months. ${ }^{12,13}$ However, androgen-deprivation therapy eventually fails and tumors may relapse, despite the absence of androgenic stimulation, and progress into the castration resistant (ie, hormone-refractory) stage, which accounts for the unappreciated failure of current therapies and the increase in prostate cancer mortality. ${ }^{12}$ On the other hand, chemotherapy usually brings drug resistance and severe adverse reactions in patients. Therefore, new anticancer drugs that can prevent the progression of prostate cancer and can execute prostate cancer cells with improved efficacy and reduced side effects are certainly and urgently needed.

Numerous abnormal biological events at cellular and subcellular levels occur in the process of prostate cancer initiation, development, progression, and relocation with the involvement of cell survival, cell death, cell invasion, activation of oncogenes, loss of tumor suppressor genes, and dysregulation of related signaling pathways. ${ }^{14-17}$ Comprehensively and globally exploring the molecule targets and underlying mechanisms will help identify new therapies for the treatment of prostate cancer. ${ }^{14,18,19}$ Recently, targeting programmed cell death and other important pathways has become a promising approach to treat prostate cancer through regulating cancer cell apoptosis and autophagy. On the other hand, emerging evidence suggests that the epithelial-mesenchymal transition (EMT) process is activated during prostate cancer development, growth, progression, and metastasis. ${ }^{20,21}$ It has been proposed that EMT is coopted by prostate cancer cells during their metastatic dissemination from a primary organ to secondary sites ${ }^{22}$ and, thus, intervention of this process may represent a novel strategy to prevent prostate cancer metastasis. Moreover, it has been reported that sirtuin (Sirt) 1, a class III nicotinamide adenine dinucleotide $\left(\mathrm{NAD}^{+}\right)$-dependent histone deacetylase, induces EMT by cooperating with EMT transcription factors and enhances prostate cancer cell migration and metastasis through deacetylation of its target proteins and modulation of EMT; ${ }^{23}$ thus, Sirt1 may represent a new therapeutic target for prostate cancer therapy.

Plumbagin ([PLB] 5-hydroxy-2-methyl-1,4-naphthoquinone, Figure 1A), an active naphthoquinone compound, possesses a wide spectrum of pharmacological activities, including anti-inflammatory, neuroprotective, anticancer, hypolipidemic, antiatherosclerotic, antibacterial, and antifungal activities in in vitro and in vivo models. ${ }^{24}$ Recently, increasing attention has been drawn to its anticancer effect. It has been proposed that the anticancer effect of PLB is mainly ascribed to induction of intracellular reactive oxygen species (ROS) generation, apoptosis and autophagy, and cell cycle arrest. ${ }^{24}$ In vitro and in vivo studies by our laboratory<smiles>CC1=CC(=O)c2c(O)cccc2C1=O</smiles>
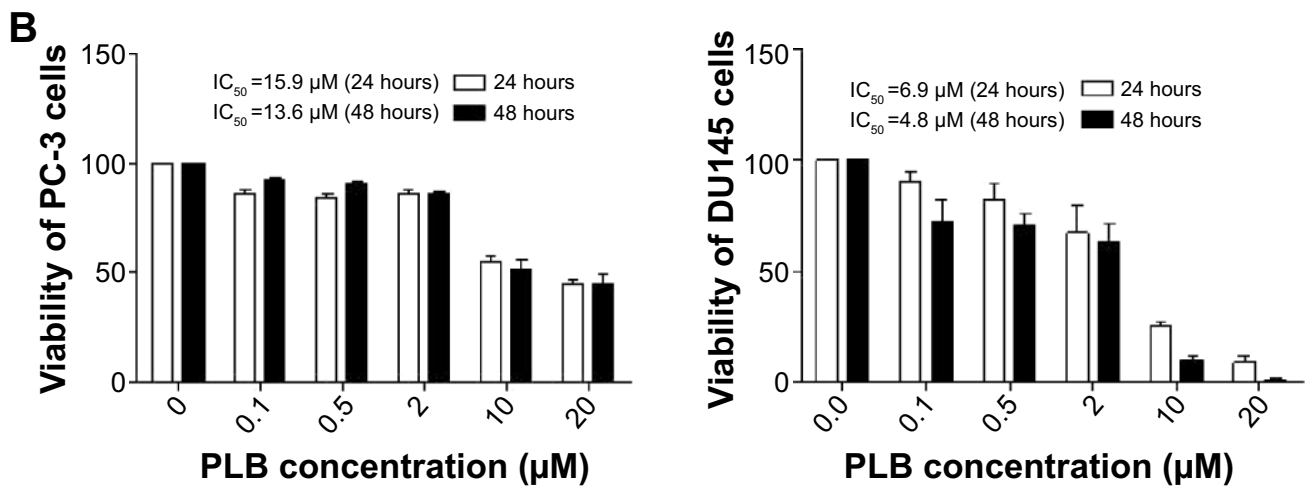

Figure I Chemical structure of PLB (5-hydroxy-2-methyl-I,4-naphthoquinone) and effect of PLB on cell viability in PC-3 and DUI45 cells.

Notes: PC-3 and DUI 45 cells were treated with PLB at 0.1 to $20 \mu \mathrm{M}$ for 24 or 48 hours. (A) Chemical structure of PLB, and (B) cell viability of PC-3 and DUI45 cells. Data are the mean \pm SD of three independent experiments.

Abbreviation: $\mathrm{IC}_{50}$, half maximal inhibitory concentration; PLB, plumbagin; SD, standard deviation. 
and other groups have showed that PLB induced cancer cell apoptosis and autophagy via modulation of cellular redox status, inhibition of NF- $\mathrm{KB}$ activation, upregulation of p53 via c-JNK phosphorylation, and inhibition of phosphatidylinositide 3-kinase (PI3K)/Akt/mTOR pathway. ${ }^{25-31}$ Several previous studies also found that ROS-mediated apoptotic pathways contributed to the anticancer effect of PLB in tumor-bearing nude mice. ${ }^{32-34}$ Although the characterization and identification of individual targets and related signaling pathways provided important evidence for the mechanism of actions of PLB in tumor cell killing in vitro and in vivo, the comprehensive and global understanding on the beneficial effect of PLB is lacking and the molecular interactome of PLB is unknown. Stable-isotope labeling by amino acids in cell culture (SILAC) is a practical and powerful approach to uncover the global proteomic responses to drug treatment and other interventions. ${ }^{35}$ In particular, it can be used to systemically and quantitatively assess the target network of drugs, evaluate drug toxicity, and identify new biomarkers for the diagnosis and treatment of important diseases such as cancer and Alzheimer's disease. ${ }^{35-37}$ In this regard, we investigated the molecular targets of PLB in prostate cancer PC-3 and DU145 cells using a combination of bioinformatic, proteomic, and functional approaches with a focus on whether there were differences in the proteomic response between the two cell lines with regard to cell cycle, apoptosis, autophagy, and EMT pathways.

\section{Materials and methods}

\section{Prediction of the interactome of PLB} and pathway analysis by molecular docking and bioinformatic approach

Protein targets were obtained from a third-party protein structure database named PDBBind. ${ }^{38}$ In this database, every ligand binding pocket is examined manually and hydrogen is added using Sybyl. According to the developer of PDBBind, the missing atoms were fixed and the amino acids residues with alternate location indicators were refined. There are a total of 1,780 Protein Data Bank (PDB) entries of human proteins available in PDBBind, and a total of 301 nonredundant PDBs corresponding to 353 ligand binding pockets were identified from it, $86 \%$ of which have resolutions of less than $2.5 \AA$. The docking boxes for each of the pockets were defined by expanding the circumscribed cube of the pocket with a margin of $8 \AA$ in six directions (up, down, front, back, left, and right).

The 2D structure of the PLB was downloaded from PubChem. The hydrogen and Gasteiger charge were added and the file format was transformed into Mol2 using Vega ZZ. The docking program AutoDock 4.2 was used to dock the PLB molecule into all 353 pockets, generating a score vector of 353 dimensions. $Z$-scores were then calculated using the methodologies we applied before. ${ }^{39-41}$ Here, an empirical threshold of -0.6 of the $Z$-score was set to indicate that the binding of PLB towards this target was likely to be true.

The Database for Annotation, Visualization and Integrated Discovery (DAVID) ${ }^{42}$ was used to provide biological functional interpretation of the potential targets of PLB derived from molecular docking calculations. UniProtKB protein IDs of these targets were converted into gene lists by using the gene accession conversion tool in the DAVID database. The DAVID database adds biological function annotation (including gene ontology, pathway, and disease association) derived from some public data sources such as Gene Ontology terms (GOTERMS) or Kyoto Encyclopedia of Genes and Genomes (KEGG) pathways. Enrichment scores and Fisher's exact test $P$-values (and corresponding false discovery rate [FDR]) were then calculated to identify which functionalrelated gene groups are significantly enriched in the target list. These significant enriched gene groups could explain the mechanism of action of PLB systematically.

\section{Chemicals and reagents}

Fetal bovine serum, PLB, dimethyl sulfoxide (DMSO), apocynin (Apo, 4'-hydroxy-3'-methoxyacetophenone, an inhibitor of nicotinamide adenine dinucleotide phosphate [NADPH] oxidase), thiazolyl blue tetrazolium bromide (MTT), Dulbecco's phosphate buffered saline (PBS), ${ }^{13} \mathrm{C}_{6}-$ L-lysine, L-lysine, ${ }^{13} \mathrm{C}_{6}{ }^{15} \mathrm{~N}_{4}$-L-arginine, and L-arginine were purchased from Sigma-Aldrich Co. (St Louis, MO, USA). Dulbecco's Modified Eagle's Medium and RPMI-1640 medium were bought from Coring Cellgro Inc. (Herndon, VA, USA). Sirtinol ([STL] a specific Sirt1 and Sirt2 inhibitor, (E)-2-((2-hydroxynaphthalen-1-yl)methyleneamino)- $N$-(1phenylethyl)benzamide) was obtained from BioVision Inc. (Milpitas, CA, USA). Western blot substrate was purchased from Thermo Fisher Scientific (Waltham, MA, USA). The polyvinylidene difluoride membrane was bought from EMD Millipore (Billerica, MA, USA). Primary antibodies against human p21 Waf1/Cip1, p27 Kip1, p53, cyclin B1, cyclin D1, cyclin-dependent kinase 1 (CDK1/CDC2/CDKN1), cyclindependent kinase 2 (CDK2/CDKN2), cytochrome c, p38 mitogen-activated protein kinase (p38 MAPK), phosphorylated (p-) p38 MAPK at Thr180/Tyr182, AMPK, p-AMPK at Thr172, protein kinase B (Akt), p-Akt at Ser473, mTOR, p-mTOR at Ser2448, PI3K, p-PI3K/p85 at Tyr458, and EMT 
antibody sampler kit (No \#9782) were all purchased from Cell Signaling Technology Inc. (Beverly, MA, USA). The EMT antibody sampler kit contains primary antibodies to N-cadherin, E-cadherin, zona occludens protein-1 (ZO-1), vimentin, slug, snail, zinc finger E-box-binding homeobox 1 (TCF8/ZEB1), and $\beta$-catenin. The antibody against human $\beta$-actin was obtained from Santa Cruz Biotechnology Inc. (Dallas, TX, USA).

\section{Cell culture and treatment}

Two human prostate cancer PC-3 and DU145 cell lines were purchased from the American Type Culture Collection (ATCC) (Manassas, VA, USA) and maintained in RPMI-1640 (PC-3 cells) and Dulbecco's Modified Eagle's Medium (DU145 cells) containing L-glutamine, phenol red, L-cysteine, L-methionine, sodium bicarbonate, and sodium pyruvate supplemented with $10 \%$ heat-inactivated fetal bovine serum at $37^{\circ} \mathrm{C}$ in a $5 \% \mathrm{CO}_{2} / 95 \%$ air humidified incubator. Cells were seeded into the plates for 24 hours to achieve a confluence of $\sim 80 \%$ prior to drug treatment. PLB was dissolved in DMSO with a stock concentration of $100 \mathrm{mM}$, and was freshly diluted to the indicated concentrations with culture medium with $0.05 \%(\mathrm{v} / \mathrm{v})$ final concentration of DMSO.

\section{Cell viability assay}

The effect of PLB on the cell viability of PC-3 and DU145 cells was examined by MTT assay. Briefly, cells were seeded into a 96-well plate at a density of 8,000 cells/well and treated with PLB at $0.1-20 \mu \mathrm{M}$ for 24 and 48 hours. After the treatment with PLB, the cells were incubated with $10 \mu \mathrm{L}$ $\left(5 \mathrm{mg} / \mathrm{mL}\right.$ ) MTT for 4 hours at $37^{\circ} \mathrm{C}$. Cell viability was determined by reduction of MTT. The absorbance was measured using a Synergy H4 Hybrid microplate reader (BioTek Inc., Winooski, VT, USA) at a wavelength of $450 \mathrm{~nm}$. The half maximal inhibitory concentration values were determined using the relative viability over PLB concentration curve.

\section{Quantitative proteomic study using SILAC}

Quantitative proteomic experiments were performed using SILAC as described previously. ${ }^{35,36,43}$ The protein quantitation kits for acidification, desalting, and digestion were purchased from Thermo Fisher Scientific. Briefly, PC-3 and DU145 cells were cultured in the medium with (heavy) or without (light) stable-isotope labeled amino acids $\left({ }^{13} \mathrm{C}_{6} \mathrm{~L}\right.$-lysine and ${ }^{13} \mathrm{C}_{6}{ }^{15} \mathrm{~N}_{4}$ L-arginine). PC-3 and DU145 cells were passaged five times by changing medium or splitting cells. Then, cells were treated with $5 \mu \mathrm{M}$ PLB for 24 hours together with stable isotope-labeled amino acids. Following that, the cell samples were harvested and lysated with hot lysis buffer (100 mM Tris base, 4\% sodium dodecyl sulfate (SDS), and $100 \mathrm{mM}$ dithiothreitol). The protein was denatured at $95^{\circ} \mathrm{C}$ for 5 minutes and sonicated at 20\% amplitude (AMPL) for 3 seconds with six pulses. After that, the samples were centrifuged at $15,000 \times g$ for 20 minutes and supernatant was collected in clean tubes. The protein concentration was determined using the Ionic Detergent Compatibility Reagent (Thermo Fisher Scientific). Subsequently, equal amounts of heavy and light protein sample were combined to reach a total volume of $30-60 \mu \mathrm{L}$ containing 300-600 $\mu$ g protein. The combined protein sample was digested using FASPTM protein digestion kit from Protein Discovery Inc. (Knoxville, TN, USA). After protein was digested, the resultant sample was acidified to a $\mathrm{pH}$ of 3 and desalted using a $\mathrm{C}_{18}$ solid-phase extraction column. The peptide mixtures were then analyzed using the hybrid linear ion trap-Orbitrap (LTQ Orbitrap XL; Thermo Fisher Scientific Inc.). The mass analysis of peptides was performed using a $10 \mathrm{~cm}$-long $75 \mu \mathrm{m}$ (inner diameter) reversed-phase column packed with $5 \mu \mathrm{m}$-diameter $\mathrm{C}_{18}$ material with $300 \AA$ pore size (New Objective, Woburn, MA, USA) with a gradient mobile phase of $2 \%-40 \%$ acetonitrile in $0.1 \%$ formic acid at $200 \mu \mathrm{L} /$ min for 125 minutes using liquid chromatography-tandem mass spectrometry (MS). The Orbitrap full MS scanning was performed at a mass $(\mathrm{m} / \mathrm{z})$-resolving power of 60,000 , with positive polarity in profile mode $\left(\mathrm{M}+\mathrm{H}^{+}\right)$. Peptide SILAC ratio was calculated using MaxQuant version 1.2.0.13. The SILAC ratio was determined by averaging all peptide SILAC ratios from peptides identified of the same protein. The protein IDs were identified using Scaffold 4.3.2 from Proteome Software Inc. (Portland, OR, USA) and the pathway was analyzed using Ingenuity Pathway Analysis (IPA) from QIAGEN (Redwood City, CA, USA).

\section{Cell cycle distribution analysis}

The effect of PLB on cell cycle of PC-3 and DU145 cells was determined using propidium iodide as the DNA stain by flow cytometry as described previously. ${ }^{44}$ Briefly, PC-3 and DU145 cells were treated with PLB at concentrations of $0.1,1,5$, and $10 \mu \mathrm{M}$ for 24 hours. In separate experiments, PC-3 and DU145 cells were treated with $5 \mu \mathrm{M}$ PLB for 4 , $8,12,24,48$, and 72 hours. Cells were trypsinized and fixed by $70 \%$ ethanol at $-20^{\circ} \mathrm{C}$ overnight. The cells were stained using $50 \mu \mathrm{g} / \mathrm{mL}$ propidium iodide. A total number of $1 \times 10^{4}$ cells was subject to cell cycle analysis using a flow cytometer (BD Biosciences, San Jose, CA, USA). 


\section{Western blotting analysis}

PC-3 and DU145 cells were washed with PBS after 24 hours' treatment with PLB at indicated concentrations, and lysed with the RIPA buffer containing protease inhibitor and phosphatase inhibitor cocktails. Protein concentrations were measured by bicinchoninic acid assay and denatured for 5 minutes at $95^{\circ} \mathrm{C}$. A quota of protein $(20 \mu \mathrm{g})$ was electrophoresed on $7 \%-12 \%$ sodium dodecyl sulfate polyacrylamide gel electrophoresis mini-gel and transferred onto methanol activated polyvinylidene difluoride membrane at $100 \mathrm{~V}$ for 2 hours at $4^{\circ} \mathrm{C}$. Membranes were probed with indicated primary antibody overnight at $4{ }^{\circ} \mathrm{C}$ and then blotted with the respective secondary antibody. Visualization was performed using BioRad system (Bio-Rad Laboratories Inc., Hercules, CA, USA). Protein level was normalized to the matching densitometric value of internal control.

\section{Measurement of intracellular ROS levels}

$\mathrm{CM}-\mathrm{H}_{2}$ DCFDA was used to measure intracellular levels of ROS according to the manufacturer's instruction. Briefly, cells were seeded into 96 -well plate $\left(1 \times 10^{4}\right.$ cells/well $)$ and treated with PLB at $0.1,1$, and $5 \mu \mathrm{M}$ for 24 hours. Following that, the cells were incubated with $5 \mu \mathrm{M} \mathrm{CM}-\mathrm{H}_{2}$ DCFDA in PBS for 30 minutes at $37^{\circ} \mathrm{C}$. In separate experiments, the intracellular ROS level was measured when cells were exposed to $5 \mu \mathrm{M}$ PLB over 72 hours. Additionally, cells were pretreated with Apo $(0.1 \mu \mathrm{M})$ for 1 hour with addition of $5 \mu \mathrm{M}$ PLB followed by further incubation for 24 hours. The fluorescence intensity was detected at wavelengths of $485 \mathrm{~nm}$ (excitation) and $530 \mathrm{~nm}$ (emission).

\section{Statistical analysis}

Data are expressed as the mean \pm standard deviation. Multiple comparisons were evaluated by one-way analysis of variance followed by Tukey's multiple comparison. A value of $P<0.05$ was considered statistically significant.

\section{Results \\ PLB likely interacts with a number of important functional proteins}

Using Vega ZZ and AutoDock 4.2 programs, we examined the interactome of PLB. There were 78 proteins that possibly interacted with PLB, including those involved in cell proliferation and apoptosis (eg, SRC, JAK2, Akt, BRAF, CDKN2A, CLK1, AURKA, and MAPK1); nucleic acid biosynthesis and metabolism (eg, GATM, MGMT, ALDH1L1, DHFR, DHODH, TYMP, TPH1, and NNMT); carbohydrate metabolism (eg, GLA, GALE, PYGL, and PYGM); amino acid and protein metabolism (eg, ASS1, BCAT2, SDS, and METAP1); phospholipid and lipid metabolism (eg, PLA2G2A and PPARA); inflammation and immune response (eg, TNFA, MASP2, and MIF); steroid metabolism and transport (AKR1C1, 1C2 and 1C3, and SHBG); blood coagulation (eg, PROCR and F9); and signal transduction (eg, ESR1, GR, PGR, and JAK2) (see Figures 2-5; Table 1). The Z'-score values were $-2.478,-2.276,-2.150,-2.084$, and -2.081 for activated CD42 kinase 1 , integrin- $\alpha-L$, Janus kinase-2 (JAK2), tyrosyl-tRNA synthetase (YARS), and tryptophan 5-hydroxylase 1 (TPH1), respectively. PLB appeared to interact with several functional protein families or subfamilies, such as the nuclear receptors (AR, GR, PGR, RARA, RARB, RARG, RXRA, RXRB, PPARA, THRB, ESR1, and ESR2), AKRs (1C1, 1C2, and 1C3), ALDHs (5 and 7), and oncoproteins and kinases (ABL, AKT, BRAF, CDKN2A, CLK1, CSNK2A1, JAK2, PAK1, MAPK1, SRC, AURKA, RPS6KA1, and MAPKAPK2). The interaction between PLB and selected targets included H-bond formation, charge interaction, and $\pi-\pi$ stacking with the involvement of a number of critical amino acid residues in the active site of targets (Table 2).

As shown in Table 3, ten functional clusters were identified to be significantly enriched (enrichment score $>3$ ) in the target list derived from molecular docking calculations. The cluster 2 is NADPH oxidation and reduction. It has been proved that PLB could bind to Nox-4, a renal NADPH oxidase, and inhibit its activity. Cluster 6 , the regulation of apoptosis, indicates that PLB could inhibit cell growth by inducing cell apoptosis.

As shown in Table 4, ten KEGG pathways significantly enriched (FDR $<0.1)$ in the target list were discovered. The first significant pathway reported by DAVID database is "Metabolism of xenobiotics by cytochrome P450" (the enrichment fold is 7.48 and FDR $=0.012$ ). Six proteins, AKR1C1, AKR1C2, AKR1C3, ADH5, ADH7, and GSTM4, were included in this pathway.

KEGG pathway analysis and the enriched gene cluster 8 (glucose metabolism) also suggested the antidiabetic effect of PLB. Seven drug targets in the insulin signaling pathway, MAP3K1, AKT1, BRAF, PYGM, GSK3B, MAPK10, and PYGL, showed high binding affinities with PLB. It agrees well with previous observations that PLB could significantly reduce the blood glucose and restore plasma insulin levels in diabetic rat models. ${ }^{45}$ Actually, PLB is isolated from the roots of Philodendron scandens and that herb is widely used to treat type II diabetes in Asia. Importantly, five of the top enriched KEGG pathways were associated with cancer. 

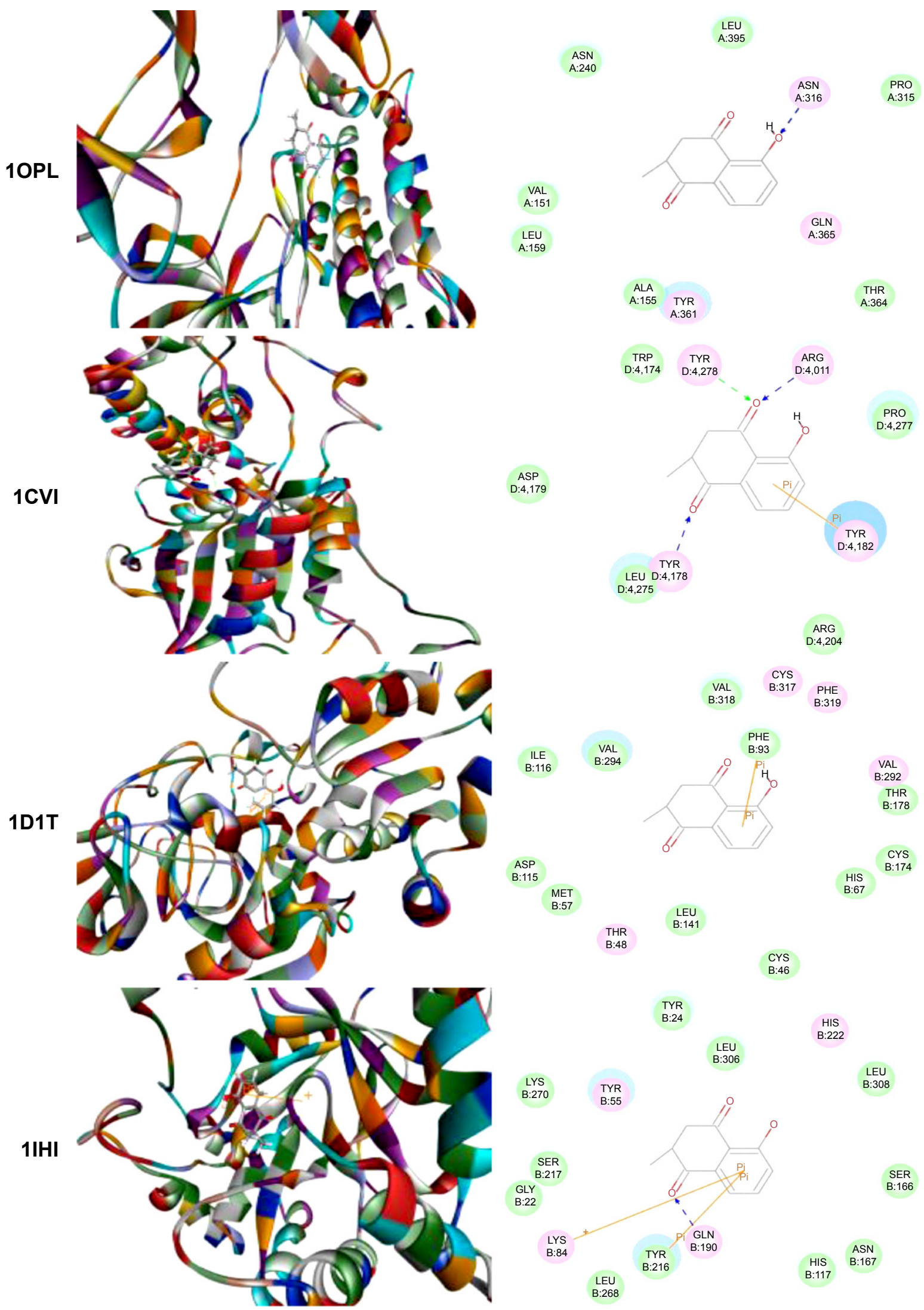

Figure 2 Molecular interactions between PLB and selected predicted targets.

Notes: Protein structure identifications from PDB. ABLI (ID: IOPL); ACPP (ID: ICVI); ADH7 (ID: IDIT); and AKRICI (ID: IIHI).

Abbreviations: ABLI, c-Abl oncogene I; ACPP, prostate acid phosphatase; ADH7, alcohol dehydrogenase 5; AKRICI, aldo-keto reductase family I, member CI; PDB, Protein Data Bank; PLB, plumbagin. 

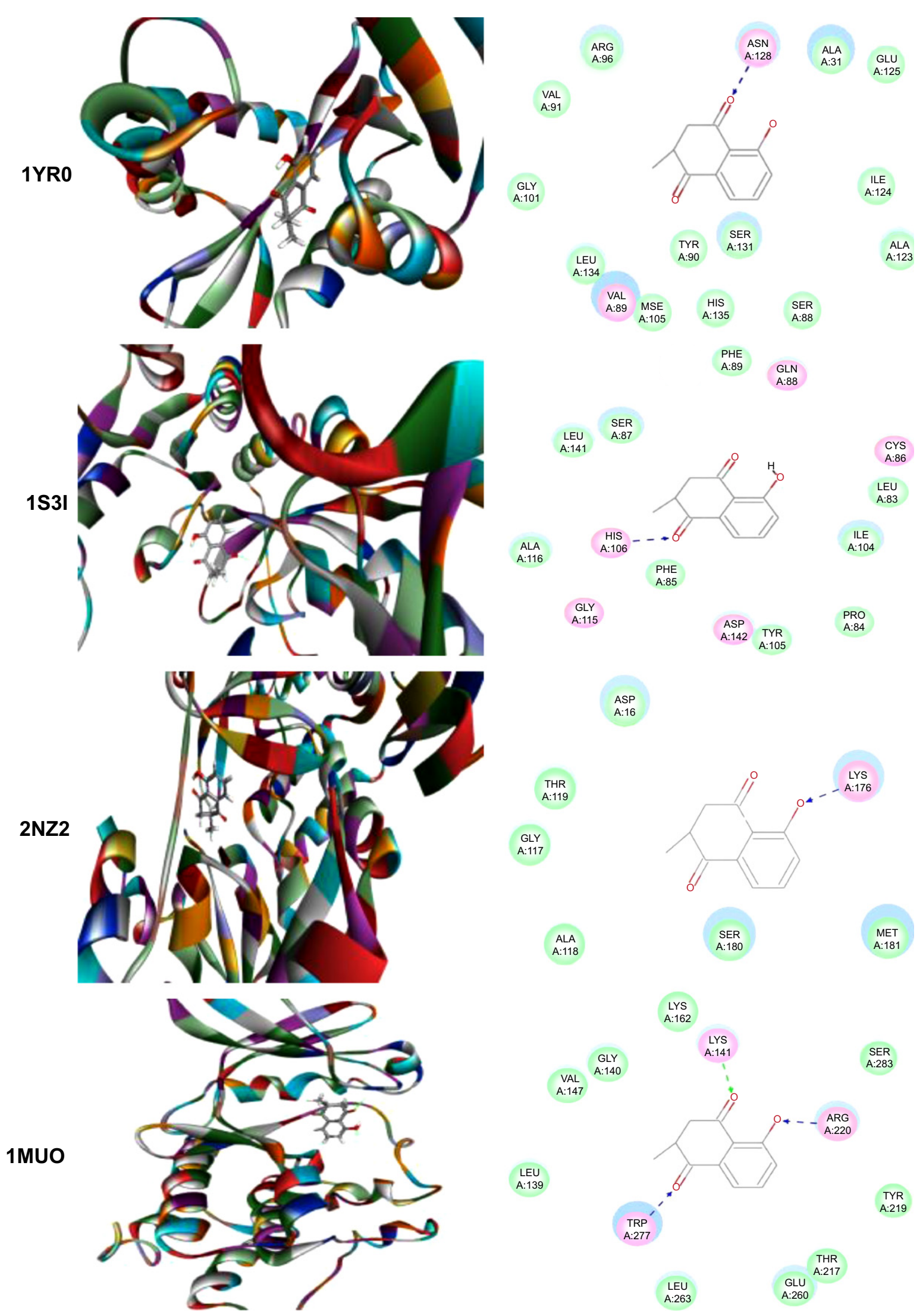

Figure 3 Molecular interactions between PLB and selected predicted targets.

Notes: Protein structure identifications from PDB. AKRIC3 (ID: IYRO); ALDHILI (ID: IS3I); ASSI (ID: 2NZ2); and AURKA (ID: IMUO).

Abbreviations: AKRIC3, aldo-keto reductase family I, member C3; ALDHILI, aldehyde dehydrogenase I family, member LI; ASSI, argininosuccinate synthase I; AURKA, aurora kinase A; PDB, Protein Data Bank; PLB, plumbagin. 


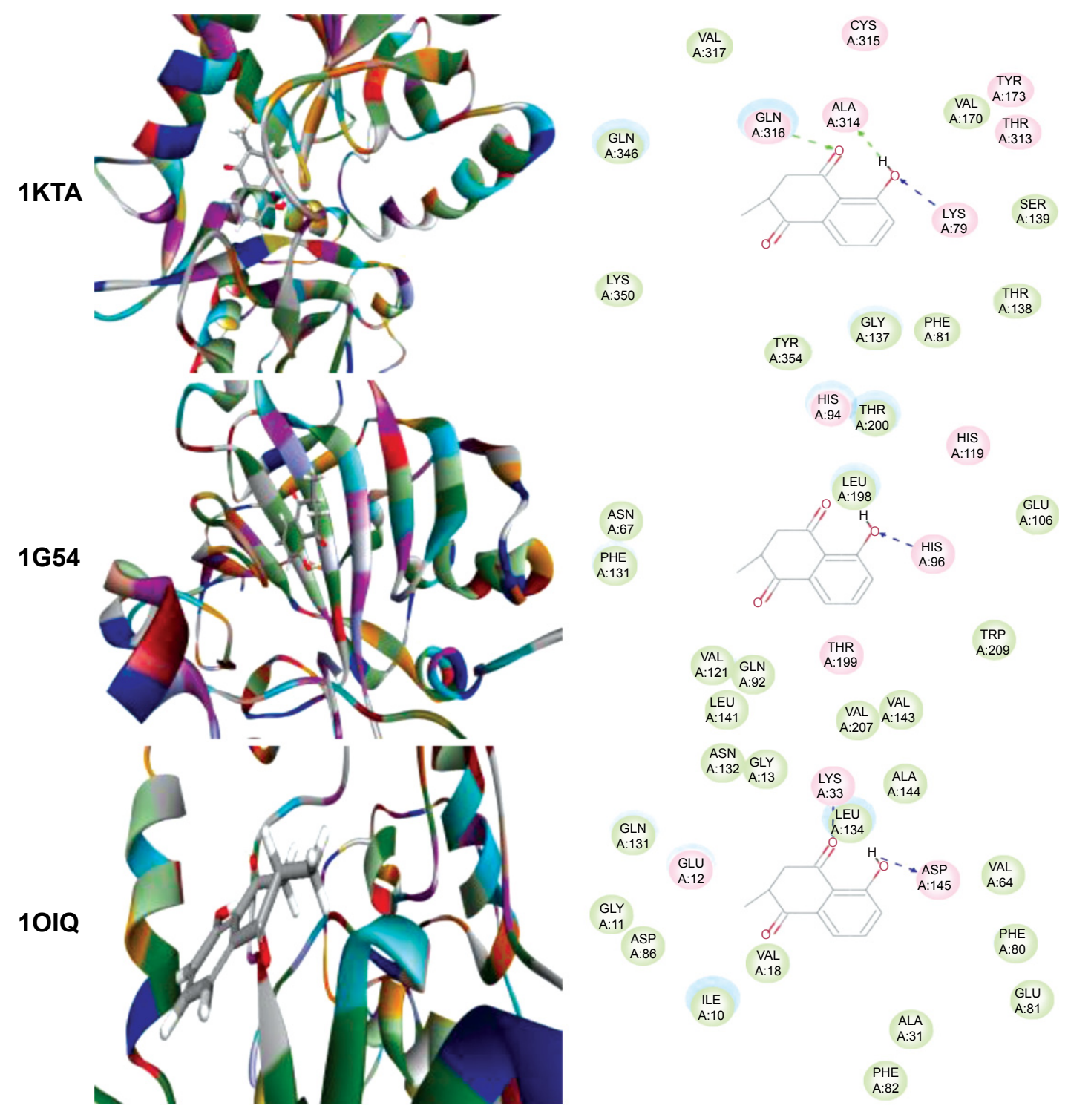

Figure 4 Molecular interactions between PLB and selected predicted targets.

Notes: Protein structure identifications from PDB. BCAT2 (ID: IKTA); CA4 (ID: IG54); and CDKN2A (ID: IOIQ).

Abbreviations: BCAT2, mitochondrial branched-chain amino-acid transaminase 2; CA4, carbonic anhydrase IV; CDKN2A, cyclin-dependent kinase inhibitor 2A; PDB, Protein Data Bank; PLB, plumbagin.

These include ErbB/EGFR/HER signaling, VEGF signaling, MAPK signaling, and colorectal cancer and prostate cancer pathways. This provides a basis for our following benchmarking experiments where PLB would be used to kill prostate cancer cells.

\section{Our proteomic study reveals that PLB regulates a large number of functional \\ proteins}

Overview of proteomic response to PLB treatment in PC-3 and DUI 45 cells

To verify the above bioinformatic data, we further carried out proteomic experiments to evaluate and compare the interactome of PLB in PC-3 and DU145 cells treated with PLB at $5 \mu \mathrm{M}$. There were 1,225 and 267 protein molecules identified as the potential targets of PLB in PC-3 and DU145 cells (Figures 6 and 7), respectively. These included a number of molecules involved in cell proliferation, cell metabolism, cell migration, cell invasion, cell survival, and cell death, such as CDK1/CDC2, MAPK, mTOR, PI3K, Akt, and E-cadherin. PLB increased the expression level of 533 protein molecules, but decreased the expression level of 682 protein molecules in PC-3 cells (Figure 6). In DU145 cells, PLB enhanced the expression of 73 protein molecules, but suppressed the expression of 193 protein molecules (Figure 7). Subsequently, these proteins were subject to IPA 


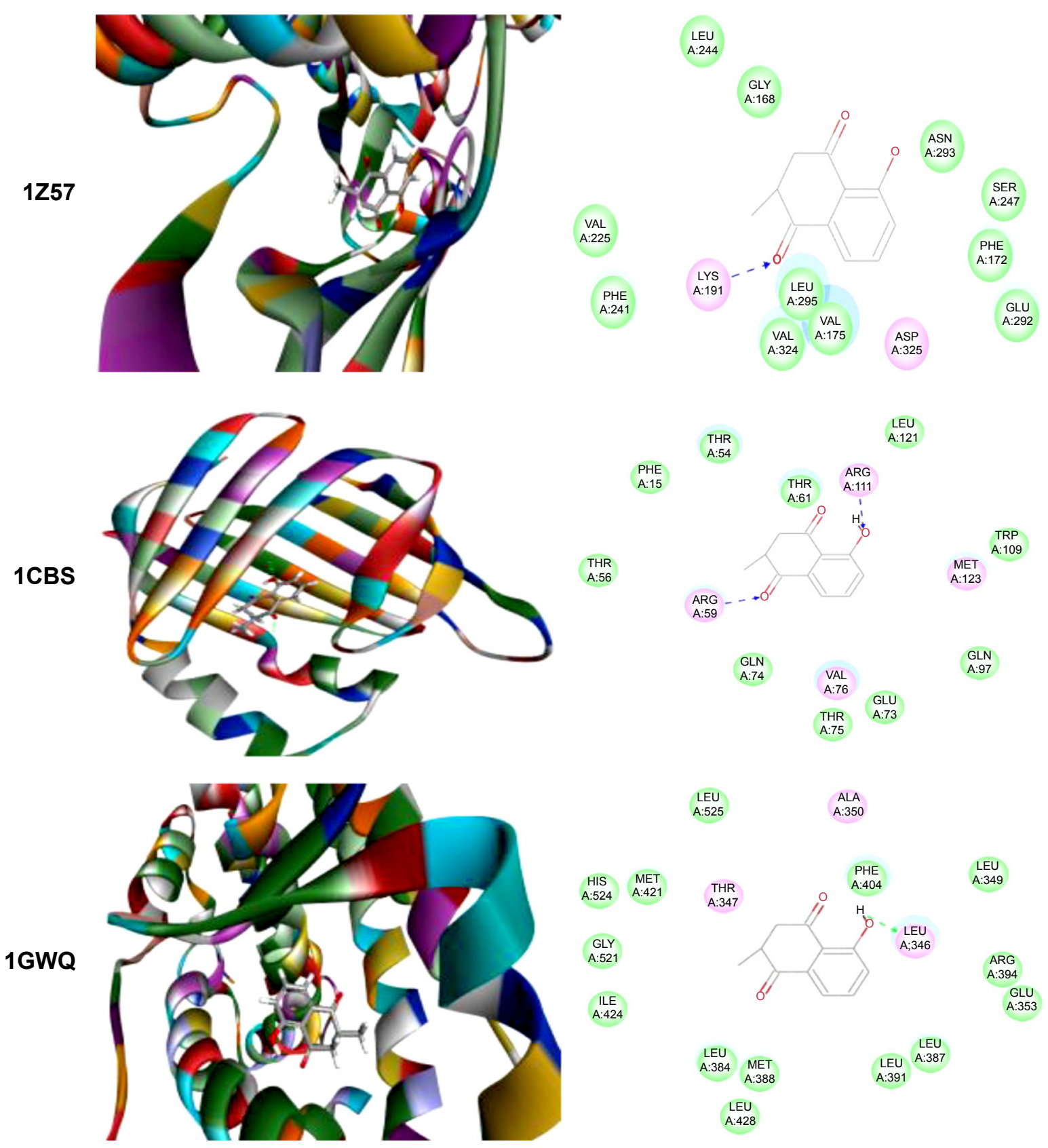

Figure 5 Molecular interactions between PLB and selected predicted targets.

Notes: Protein structure identifications from PDB. CLKI (ID: IZ57); CRABP2 (ID: ICBS); and ESR I/NR3AI (ID:IGWQ).

Abbreviations: CLKI, CDC-like kinase I; CRABP2, cellular retinoic acid binding protein 2; ESRI/NR3AI, estrogen receptor- $\alpha$; PDB, Protein Data Bank; PLB, plumbagin.

pathway analysis. As shown in Figures 8 and 9 and Tables 5 and 6,341 and 107 signaling pathways and cellular functions were potentially regulated by PLB in PC-3 and DU145 cells, respectively.

\section{PLB regulates cell cycle regulators of PC-3 cells}

It has been reported that PLB-induced cell cycle arrest is an important contributor to PLB's anticancer effect. ${ }^{30,46} \mathrm{We}$ treated PC-3 and DU145 cells with $5 \mu \mathrm{M}$ PLB for 24 hours and then cell samples were subject to quantitative proteomic analysis. The results showed that PLB regulated cell cycle at $\mathrm{G}_{1} / \mathrm{S}$ and $\mathrm{G}_{2} / \mathrm{M}$ DNA damage checkpoints in PC-3 cells with the involvement of a number of functional proteins (Table 5). These included RPL11, RPL5, HDAC2, PA2G4, GNL3, and SKP1 at $\mathrm{G}_{1} / \mathrm{S}$ checkpoint and YWHAQ, PRKDC, YWHAG, YWHAE, YWHAH, YWHAB, YWHAZ, SFN, 


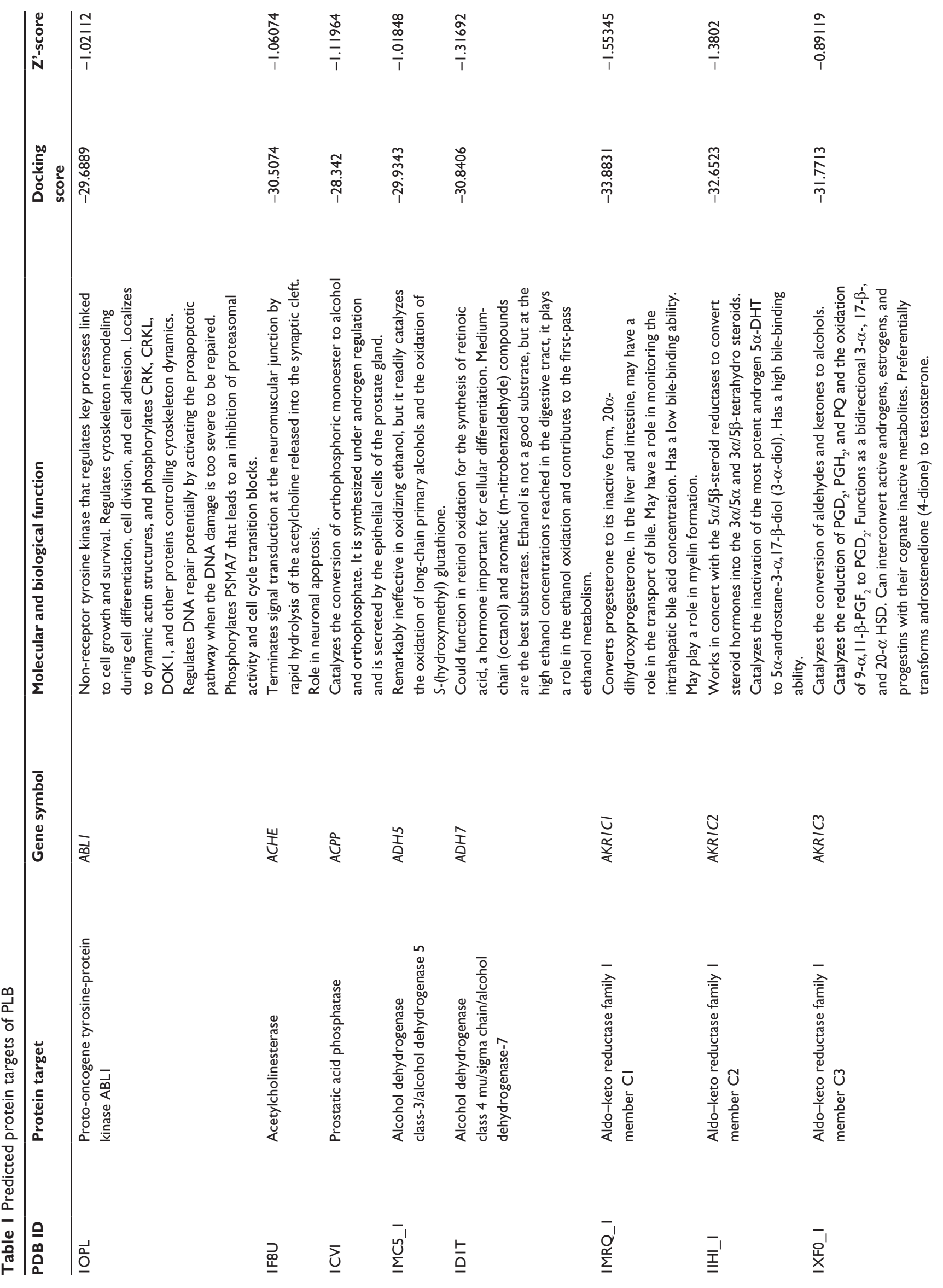


芩

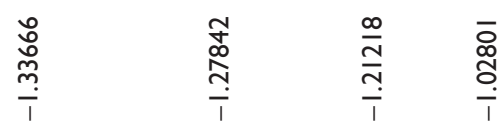

$\frac{\hat{\underline{0}}}{\frac{\hat{n}}{i}}$

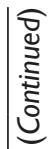

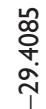

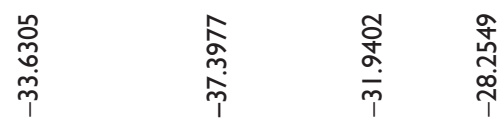

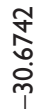

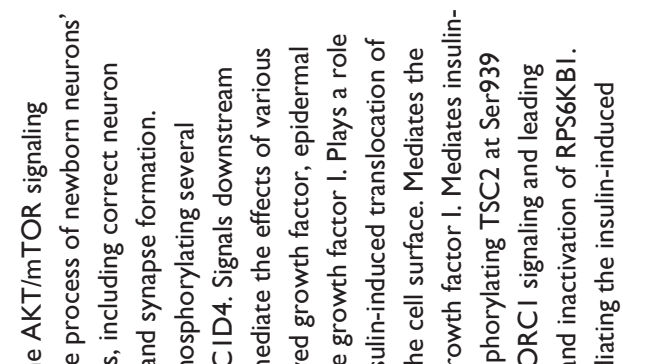

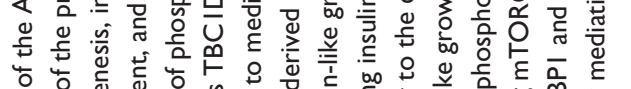

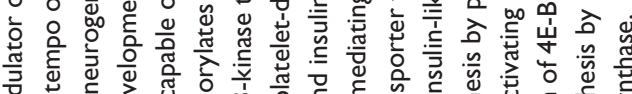

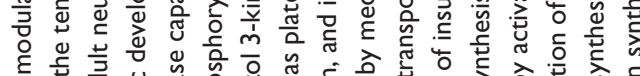

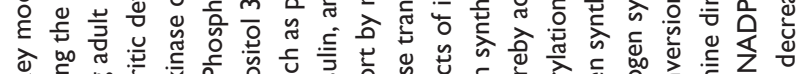

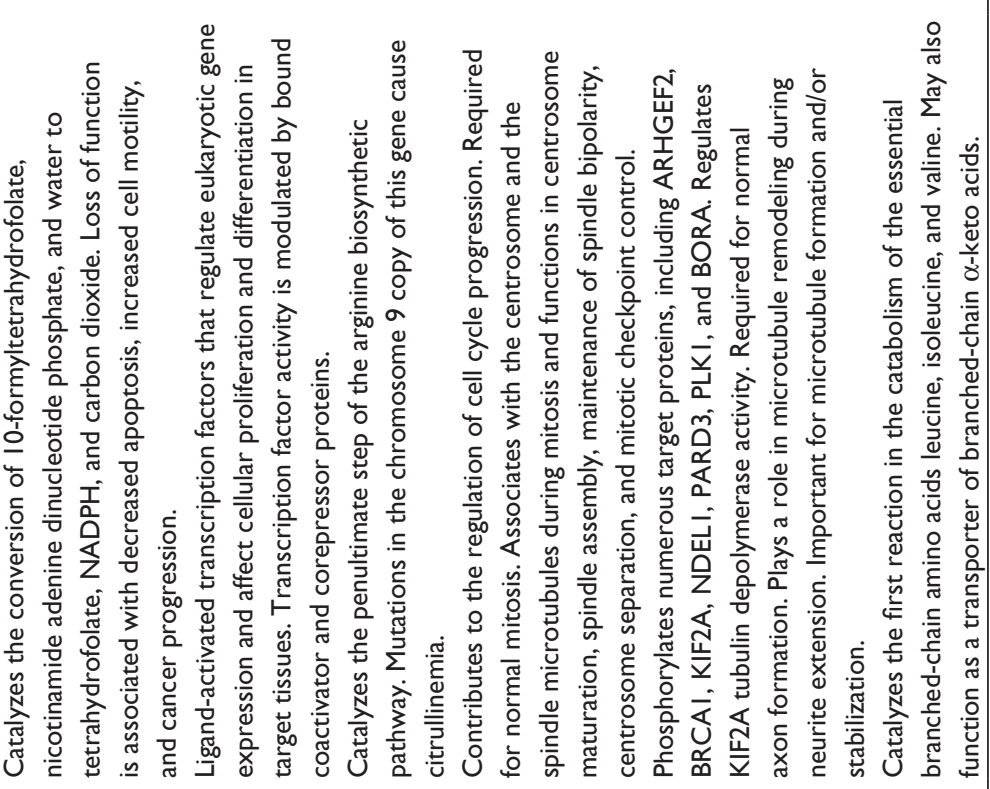

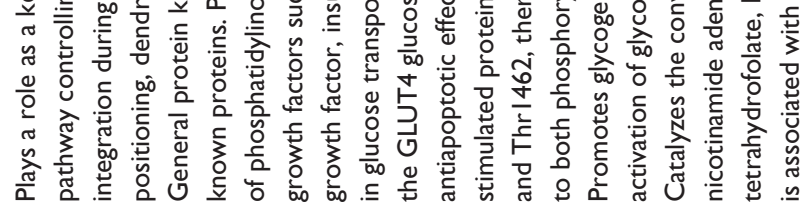

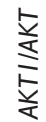

ఏ

空

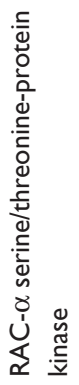
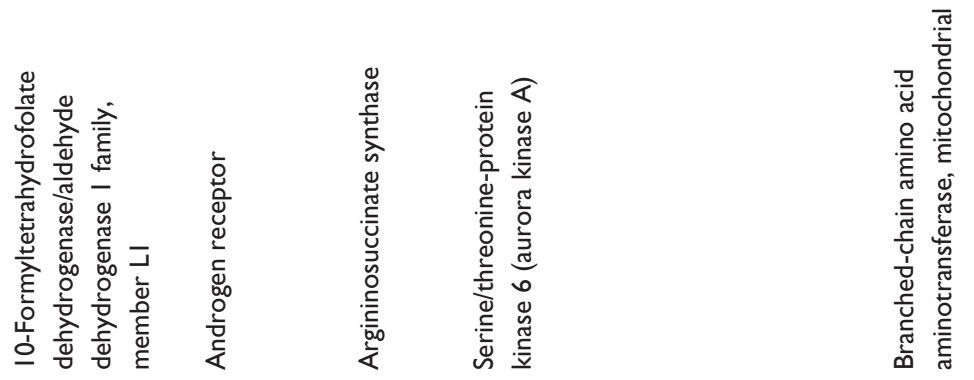

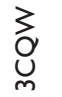

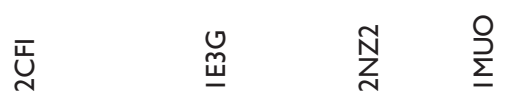

$\stackrel{E}{\underline{E}}$ 


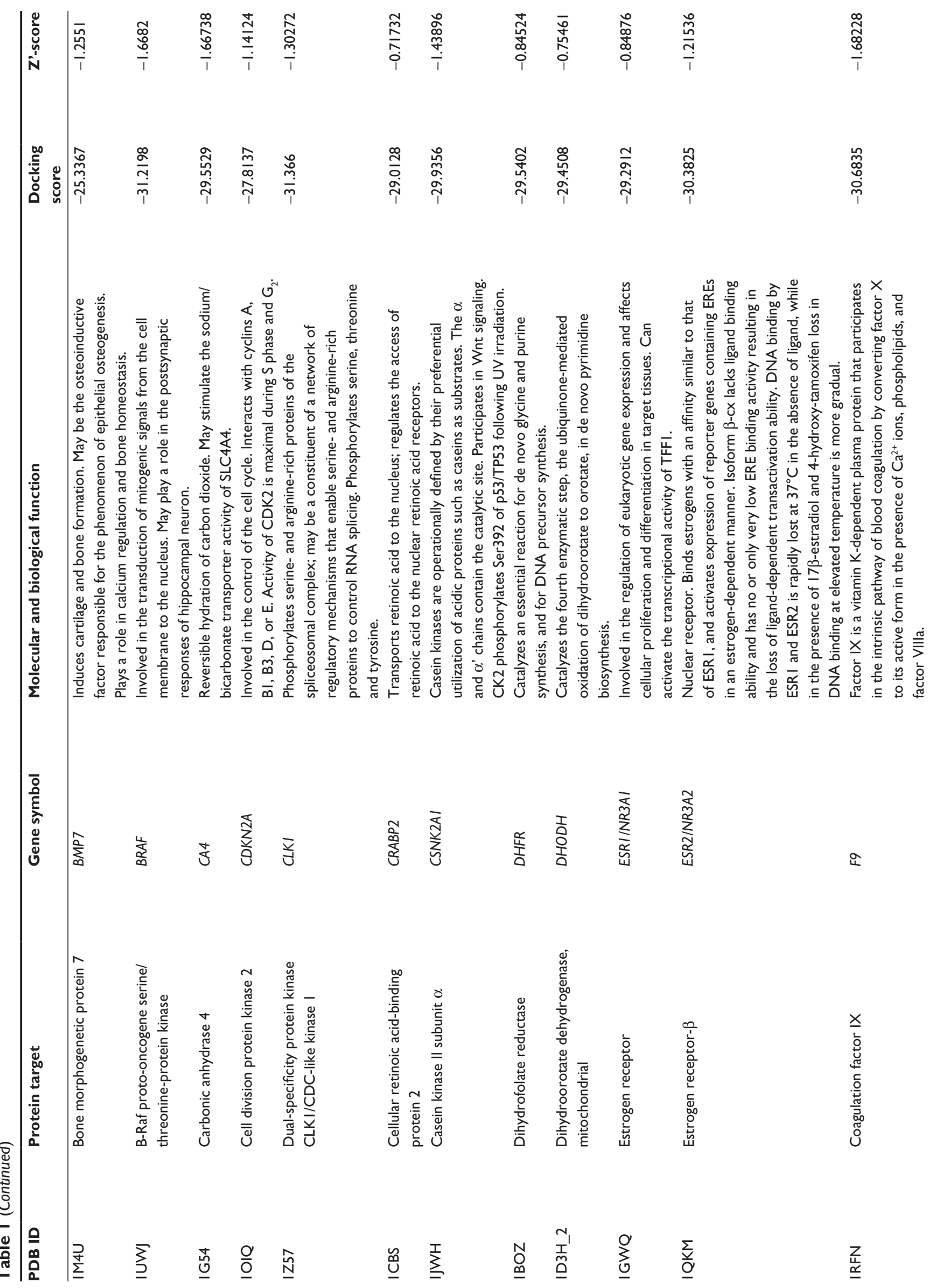




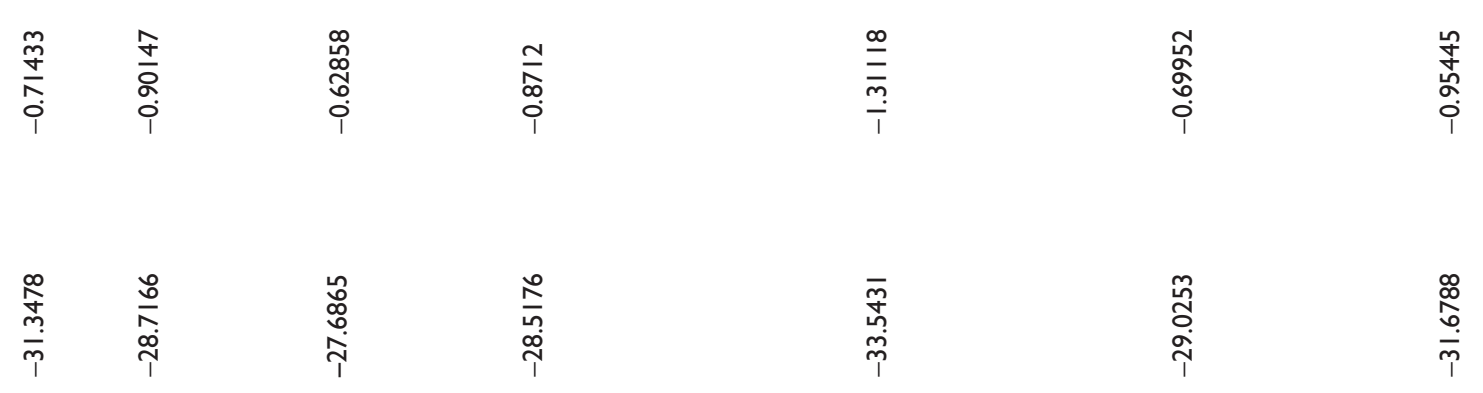

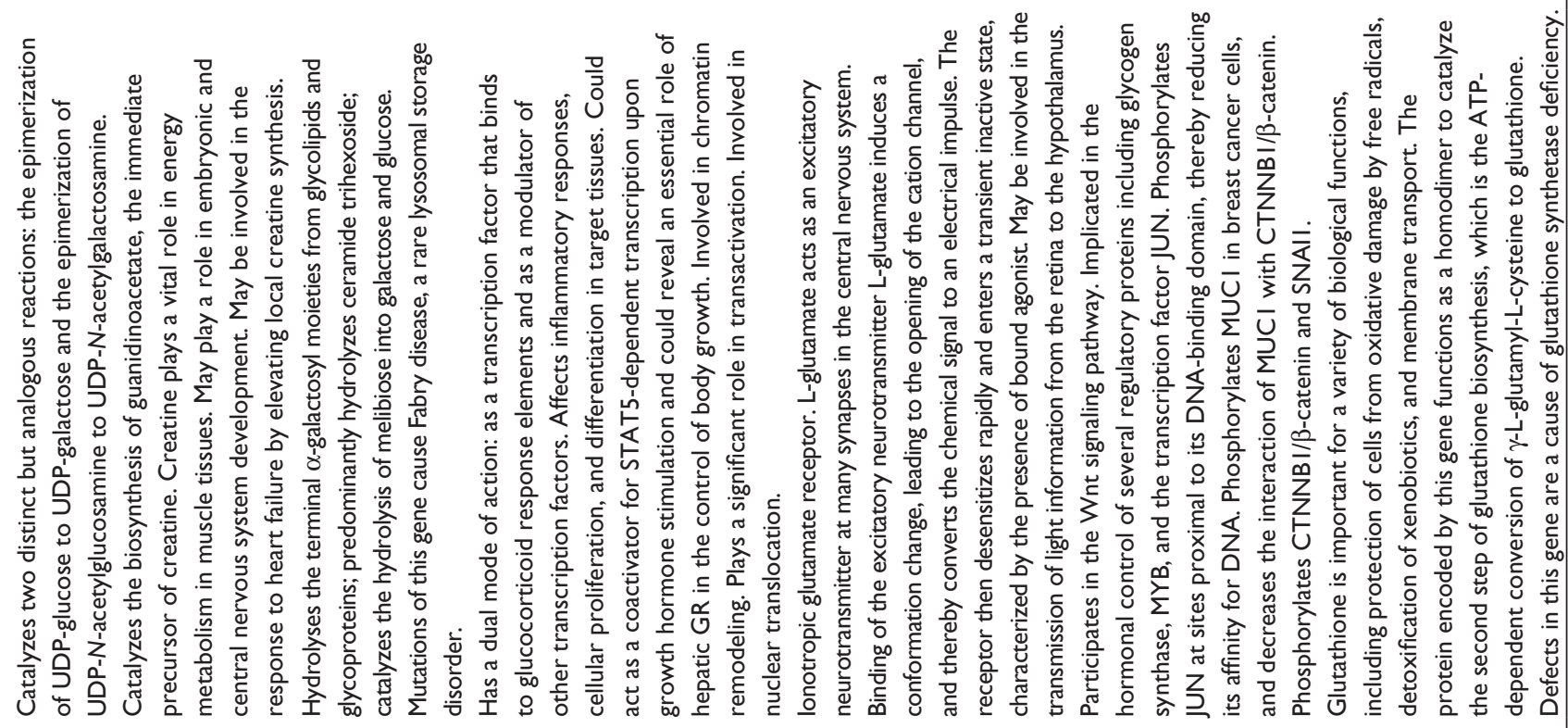

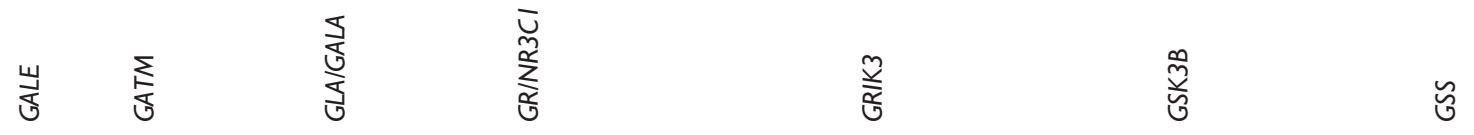

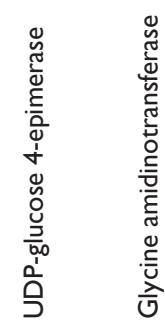

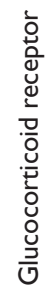

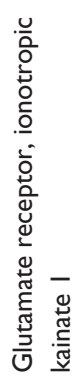

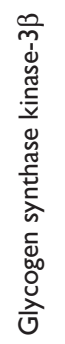

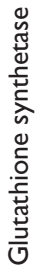

章怘

$\stackrel{\stackrel{N}{\underline{y}}}{\underline{\underline{Y}}}$

$\underset{\mathrm{N}}{\mathrm{Z}}$

$\cong$

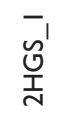




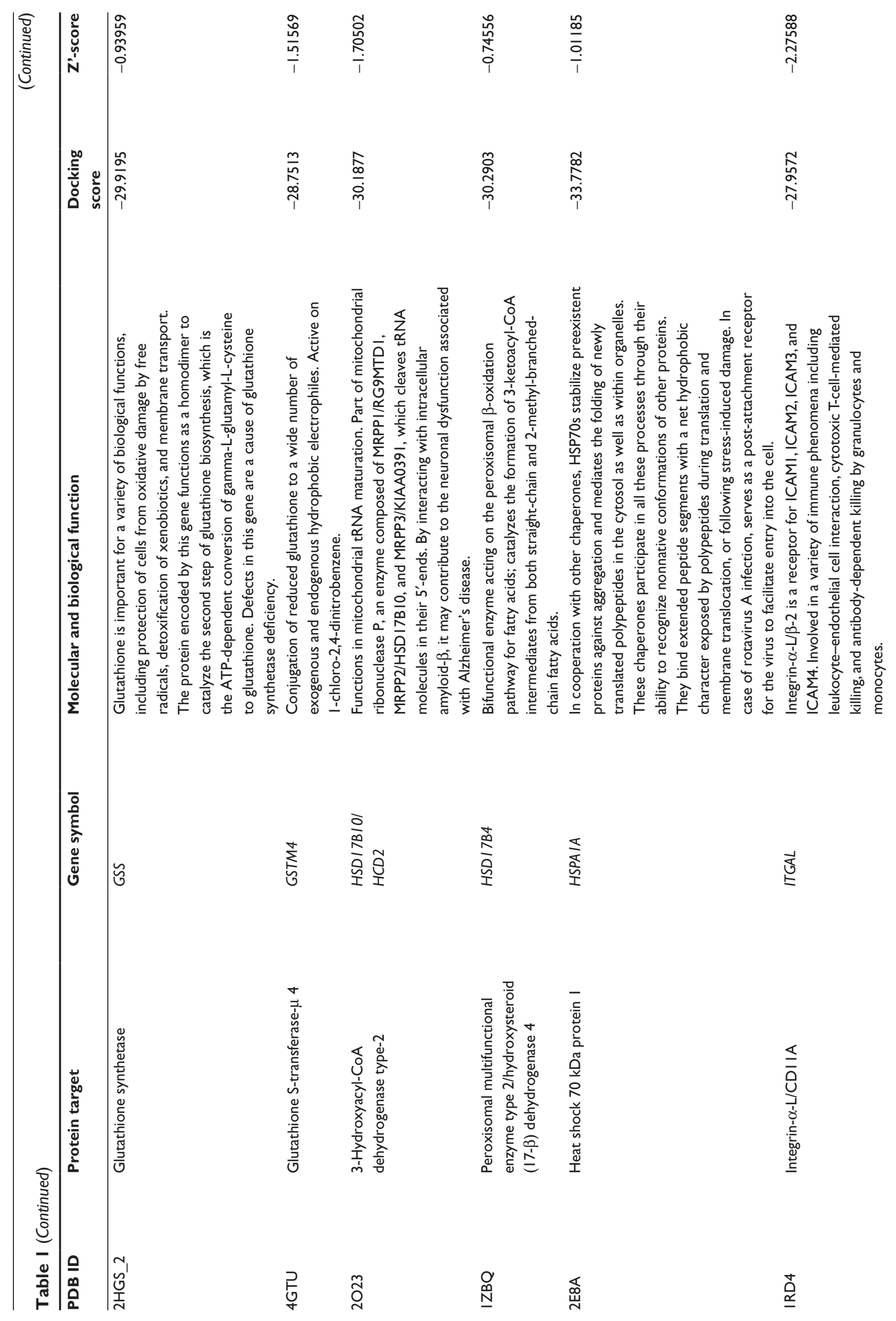


ํํำ

$\underset{\substack{q \\ \hat{i}}}{\hat{i}}$

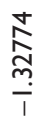

$\underset{\substack{\infty \\ \stackrel{\leftrightarrow}{n}}}{i}$
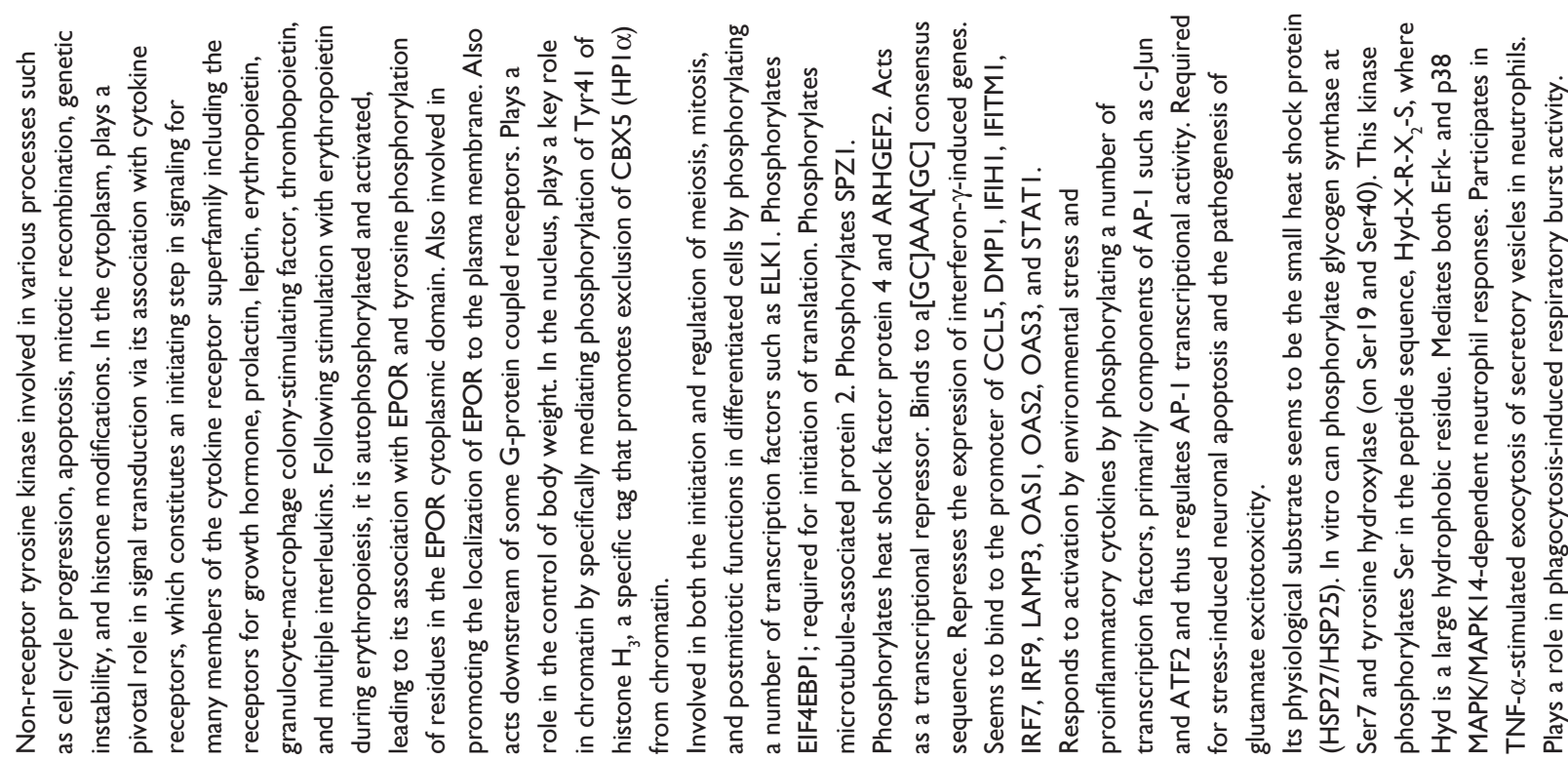

䒚

$\frac{\text { 盖 }}{\frac{\underline{\underline{a}}}{\Sigma}}$

$\frac{\substack{\frac{n}{2} \\ \frac{2}{2}}}{\frac{2}{2}}$

$\frac{\frac{2}{2}}{\frac{2}{2}}$

$\frac{\bar{v}}{\frac{0}{5}}$

$\frac{\frac{0}{v}}{\frac{v}{L}}$

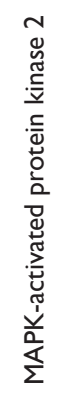

莡

$\stackrel{O}{\underline{I}}$

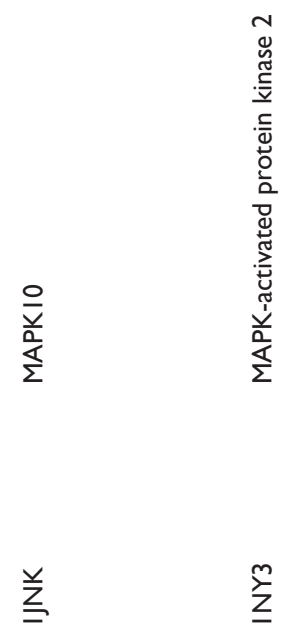




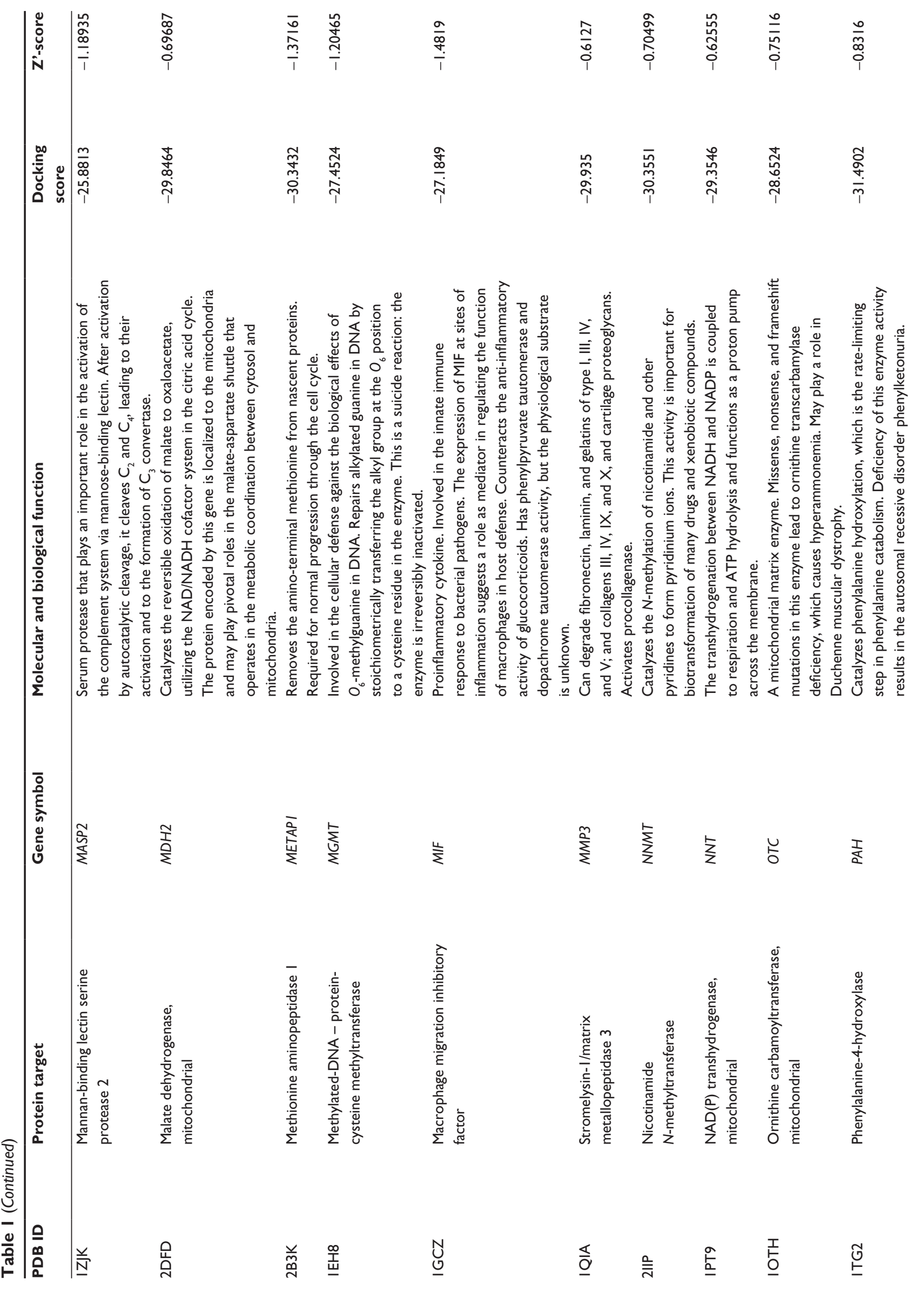




\begin{tabular}{|c|c|c|c|c|c|c|}
\hline 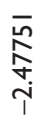 & $\begin{array}{l}\text { 足 } \\
\hat{\alpha} \\
\text { o } \\
\end{array}$ & 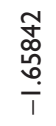 & 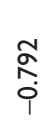 & 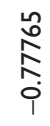 & $\begin{array}{l}\infty \\
\stackrel{\infty}{\infty} \\
\stackrel{0}{0} \\
\stackrel{0}{0}\end{array}$ & 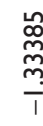 \\
\hline & $\begin{array}{l}\text { ণે } \\
\text { ¿্ల }\end{array}$ & $\begin{array}{l}\text { م } \\
\hat{0} \\
\stackrel{\alpha}{\alpha}\end{array}$ & 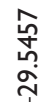 & $\begin{array}{l}\hat{f} \\
\hat{f} \\
\text { d }\end{array}$ & 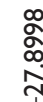 & $\begin{array}{l}\stackrel{n}{\approx} \\
\infty \\
\infty \\
0\end{array}$ \\
\hline
\end{tabular}

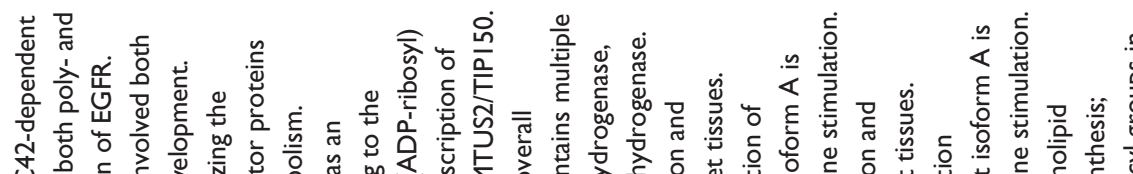

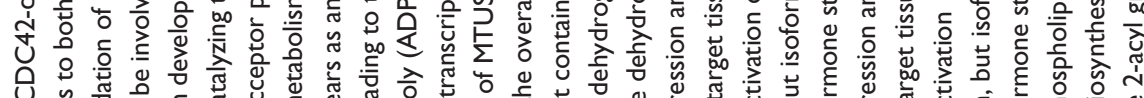

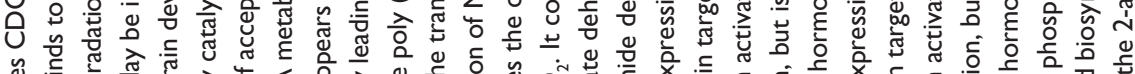

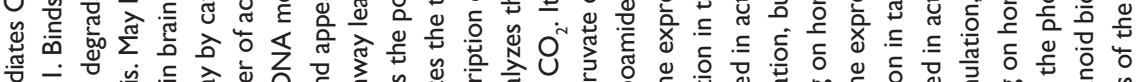

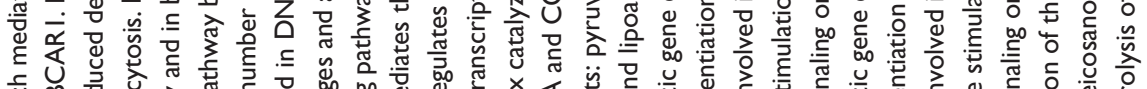

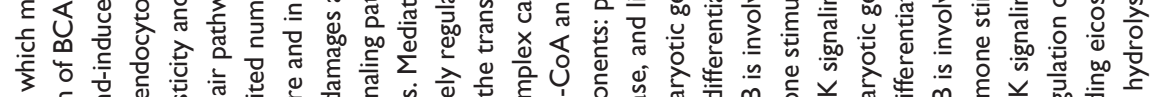

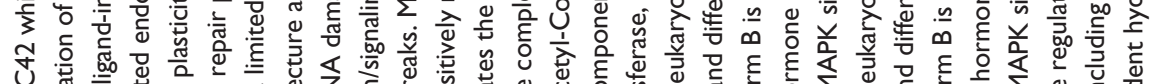

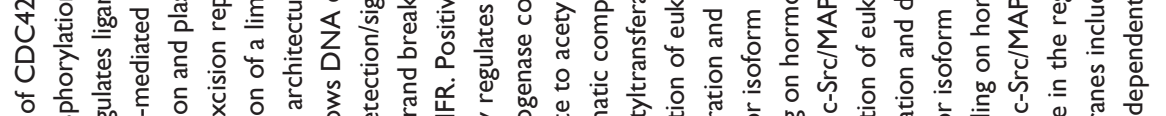

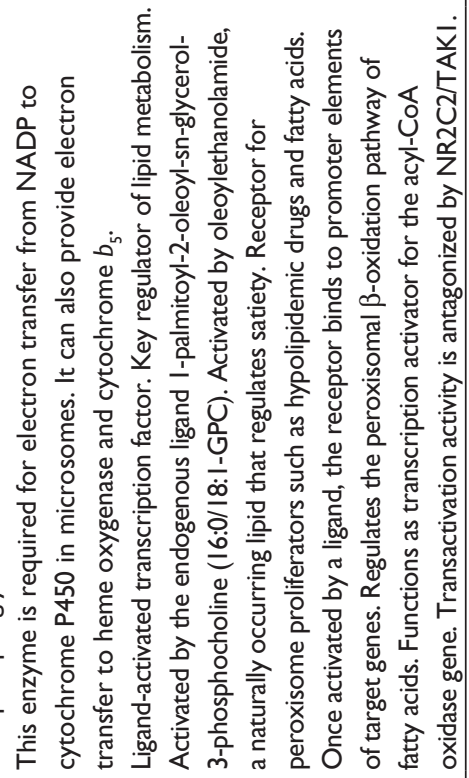

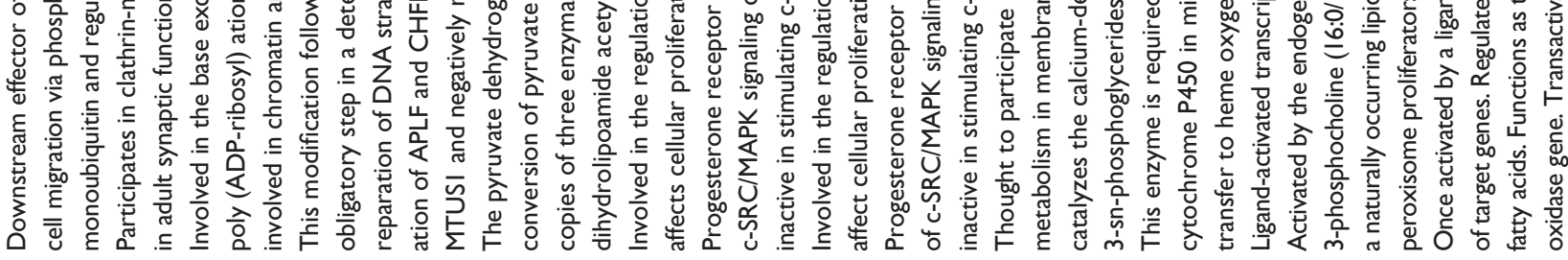
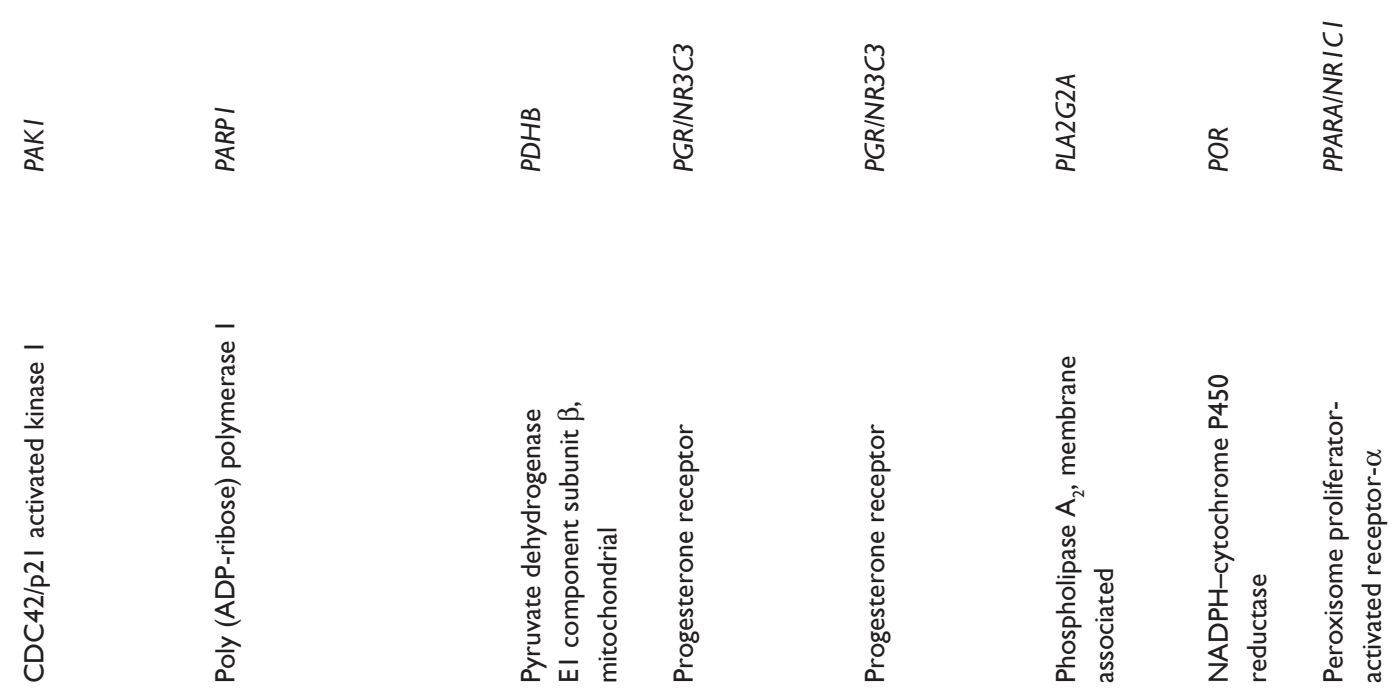

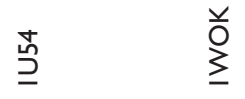
$\stackrel{\stackrel{t}{Z}}{\underline{N}}$

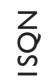

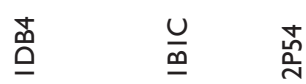




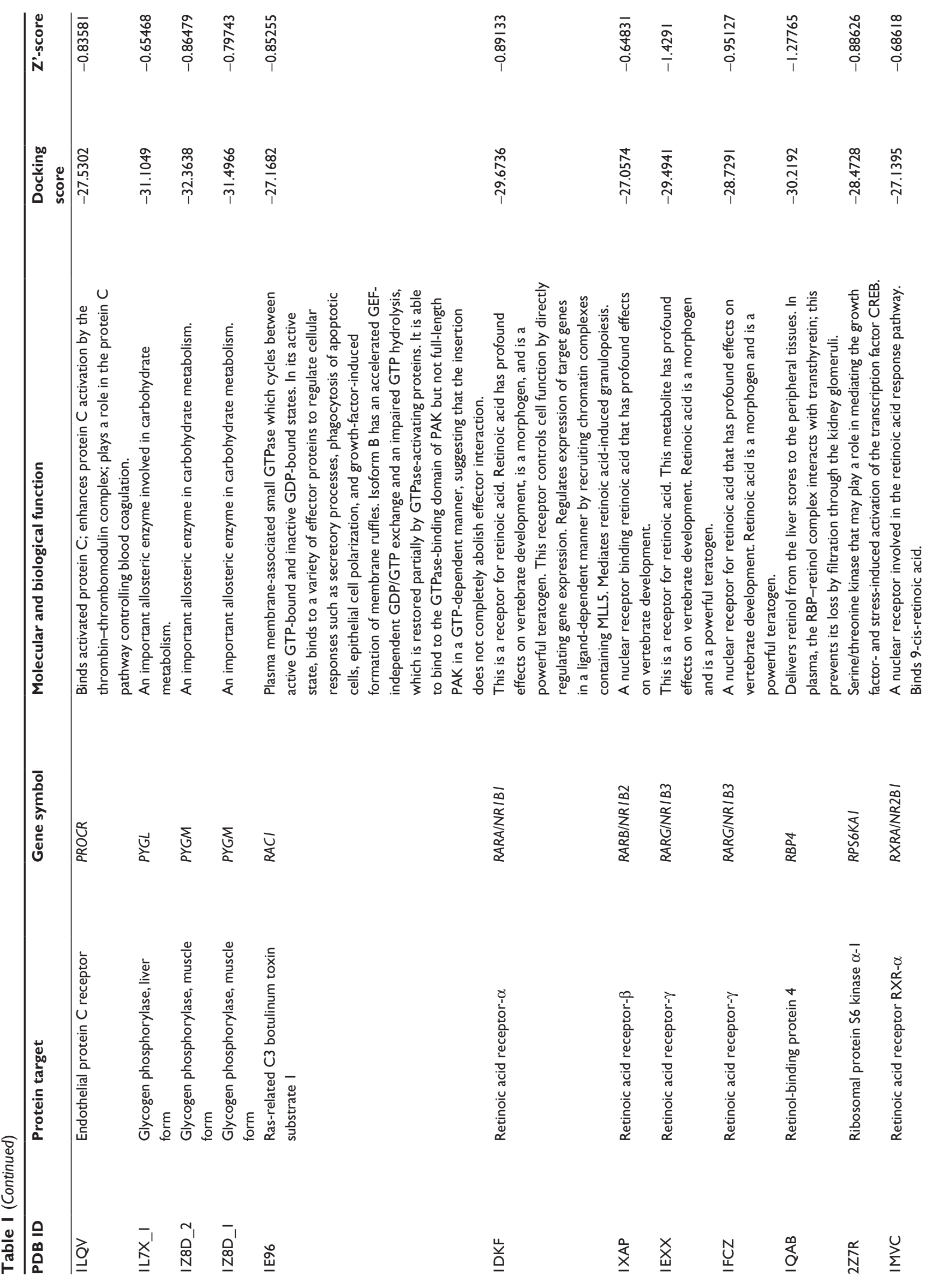




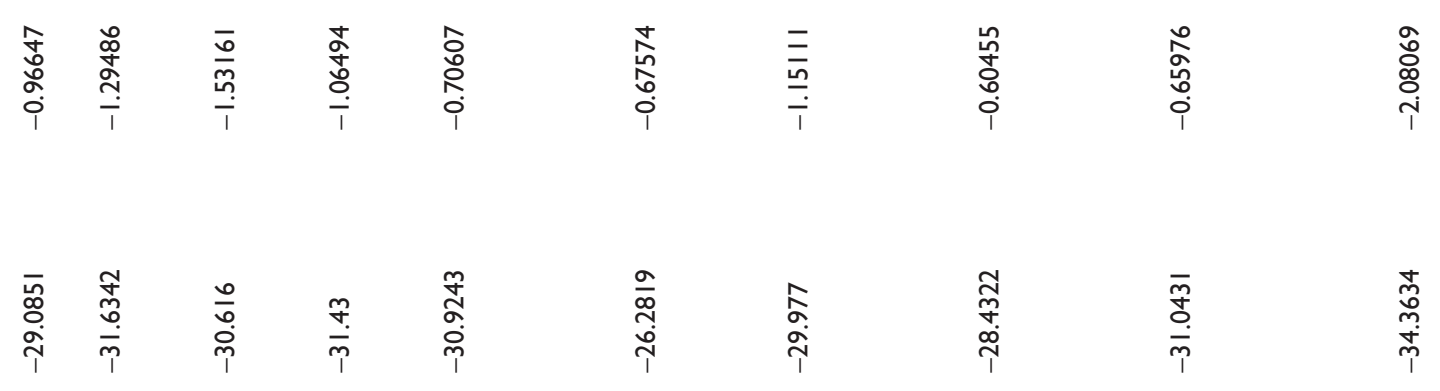
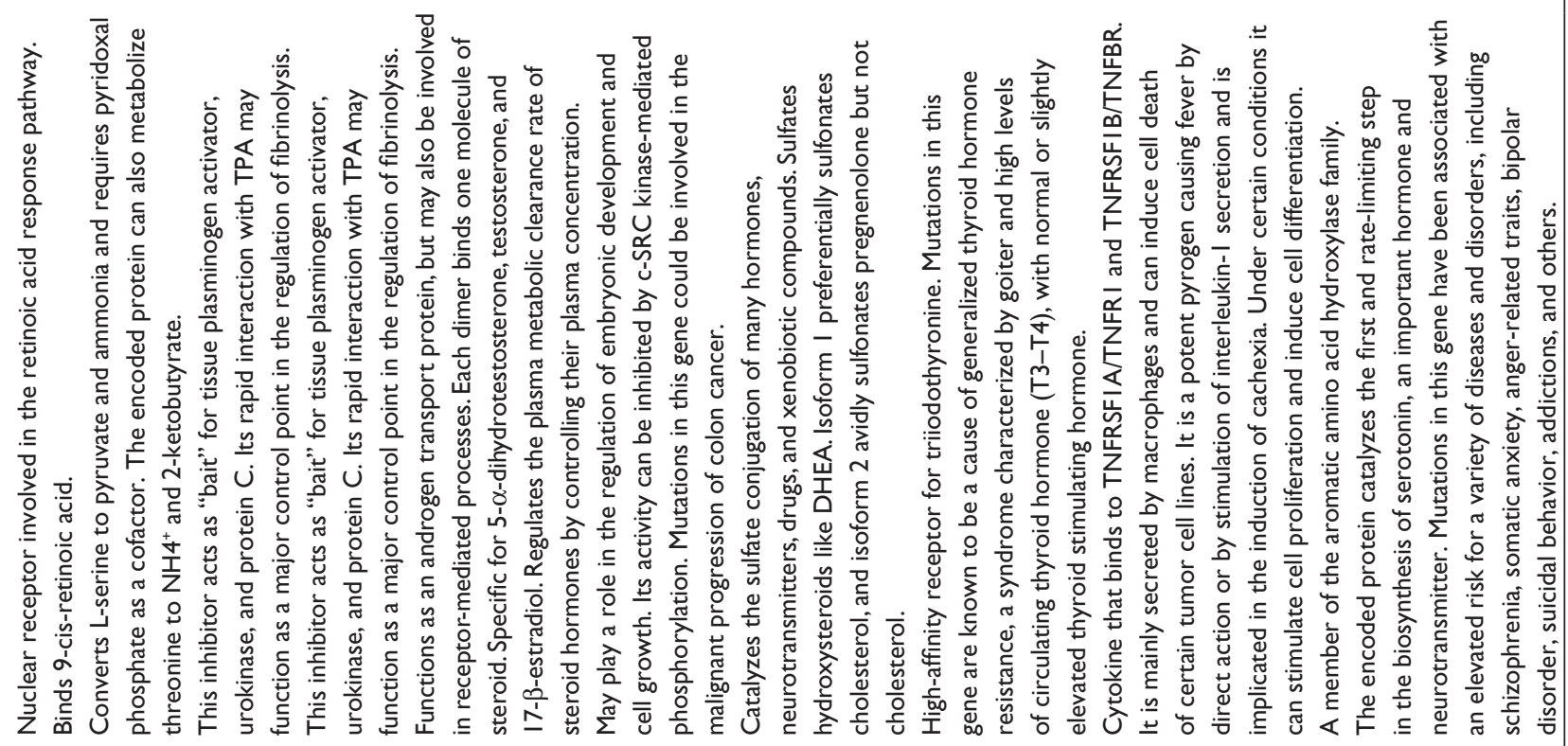

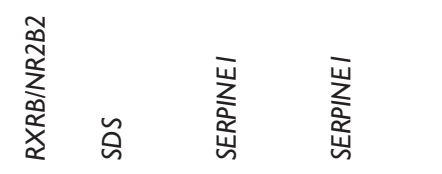

量

高峷

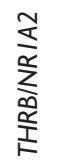

$\underset{k}{\stackrel{k}{k}}$

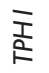

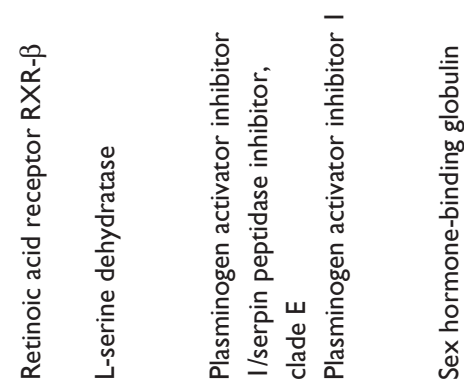

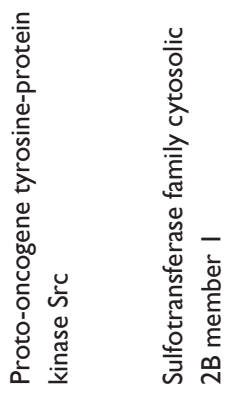

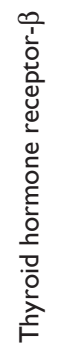

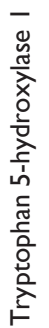

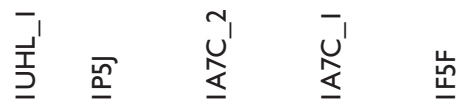

$\stackrel{\underline{N}}{\underline{O}}$

胥

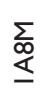

$\underset{\underline{\Sigma}}{\underline{\Sigma}}$ 


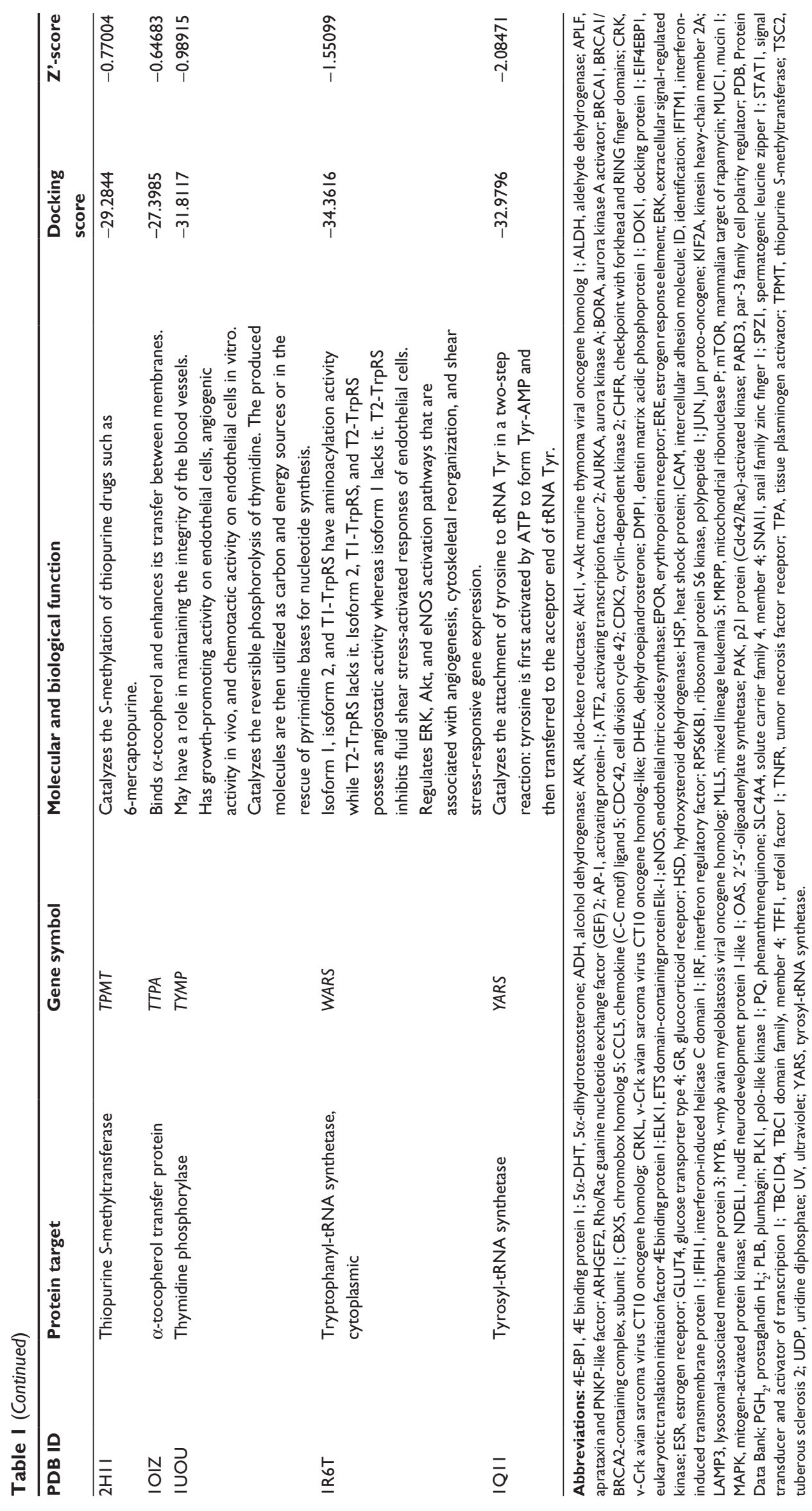


Table 2 Molecular interactions of PLB with selected potential target proteins

\begin{tabular}{|c|c|c|c|c|c|c|c|c|}
\hline $\begin{array}{l}\text { Target } \\
\text { protein }\end{array}$ & PDB ID & $\begin{array}{l}\text { CDOCKER } \\
\text { interaction energy } \\
\text { (CIE kcal/mol) }\end{array}$ & $\begin{array}{l}\text { H-bond } \\
\text { number }\end{array}$ & $\begin{array}{l}\text { Residues involved } \\
\text { in } \mathrm{H} \text {-bond } \\
\text { formation }\end{array}$ & $\begin{array}{l}\text { Charge } \\
\text { interactions }\end{array}$ & $\begin{array}{l}\text { Residues } \\
\text { involved in charge } \\
\text { interactions }\end{array}$ & $\begin{array}{l}\pi-\pi \\
\text { stacking }\end{array}$ & $\begin{array}{l}\text { Residues } \\
\text { involved in } \\
\pi-\pi \text { stacking }\end{array}$ \\
\hline$\overline{\mathrm{ABLI}}$ & IOPL & 18.9346 & 1 & O-Asn316 & 0 & - & 0 & - \\
\hline ACPP & $I C V I$ & 26.6927 & 3 & $\begin{array}{l}\text { O-Arg40II, } \\
\text { O-Tyr4I78, } \\
\text { O-Tyr4278 }\end{array}$ & 0 & - & 1 & Tyr4I82 \\
\hline ADH5 & $\mathrm{IM} 6 \mathrm{H}$ & I8.8434 & 0 & - & 0 & - & 0 & - \\
\hline ADH7 & IDIT & 22.8913 & 0 & - & 0 & - & 1 & Phe93 \\
\hline AKRICI & $\mathrm{IIHI}$ & 24.3975 & I & O-Gln & I & Lys84 & 1 & Tyr216 \\
\hline AKRIC3 & IYRO & 25.8425 & I & O-Asn I 28 & 0 & - & 0 & - \\
\hline Aktl/Akt & $3 \mathrm{CQW}$ & 24.9918 & 0 & - & 0 & - & 0 & - \\
\hline ALDHILI & $|S 3|$ & 26.7855 & I & O-His I06 & 0 & - & 0 & - \\
\hline AR/NR3C4 & IE3G & 28.3581 & 0 & - & 0 & - & 0 & - \\
\hline ASSI & $2 N Z 2$ & 19.5889 & 1 & O-Lys I76 & 0 & - & 0 & - \\
\hline AURKA & IMUO & 24.3512 & 3 & $\begin{array}{l}\text { O-Lys 14I, } \\
\text { O-Arg220, } \\
\text { O-Trp277 }\end{array}$ & 0 & - & 0 & - \\
\hline BCAT2 & IKTA & 25.82 & 3 & $\begin{array}{l}\text { H-Ala314, } \\
\text { O-Lys79, } \\
\text { O-Gln316 }\end{array}$ & 0 & - & 0 & - \\
\hline BMP7 & IM4U & 19.8572 & 0 & - & 0 & - & 0 & - \\
\hline BRAF & IUWJ & 23.1585 & 0 & - & 0 & - & 0 & - \\
\hline CA4 & IG54 & 27.6704 & I & O-His96 & 0 & - & 0 & - \\
\hline CDKN2A & IOIQ & 31.8477 & 2 & $\begin{array}{l}\text { H-Asp I45, } \\
\text { O-Lys33 }\end{array}$ & 0 & - & 0 & - \\
\hline CLKI & IZ57 & 29.7806 & I & O-Lys191 & 0 & - & 0 & - \\
\hline CRABP2 & ICBS & 25.3587 & 2 & $\begin{array}{l}\text { O-Arg55, } \\
\text { O-ArgIII }\end{array}$ & 0 & - & 0 & - \\
\hline ESRI/NR3AI & IGWQ & 26.8968 & I & H-Leu346 & 0 & - & 0 & - \\
\hline ESR2/NR3A2 & IQKM & 28.2648 & 0 & - & 0 & - & 0 & - \\
\hline
\end{tabular}

Abbreviations: ABLI, c-abl oncogene I; ACPP, prostate acid phosphatase; ADH, alcohol dehydrogenase; AKR, aldo-keto reductase; Akt, v-Akt murine thymoma viral oncogene homolog; ALDH, aldehyde dehydrogenase; AR, androgen receptor; ASS, argininosuccinate synthase; AURKA, aurora kinase A; BCAT, mitochondrial branchedchain amino-acid transaminase; BMP, bone morphogenetic protein; BRAF, v-Raf murine sarcoma viral oncogene homolog B; CA, carbonic anhydrase; CDKN, cyclin-dependent kinase inhibitor; CLK, CDC-like kinase; CRABP, cellular retinoic acid binding protein; ESR, estrogen receptor; ID, identification; PDB, Protein Data Bank; PLB, plumbagin.

$\mathrm{SKP} 1$, and $\mathrm{CDK} 1$ at $\mathrm{G}_{2} / \mathrm{M}$ checkpoint (Figure 10). However, the proteomic analysis did not reveal any remarkable effect of PLB on proteins that regulate cell cycle in DU145 cells.

\section{PLB regulates apoptosis and autophagy in PC-3 and DUI45 cells}

Apoptosis and autophagy are two predominant programmed cell death pathways and they have been considered to be promising targets for the treatment of cancer via regulating mitochondria-dependent, mitochondria-independent, or PI3K/ Akt/mTOR-mediated pathways. ${ }^{47-51}$ As shown in Tables 5 and 6 , PLB regulated apoptotic signaling pathway and mitochondrial function involving a number of functional proteins. These included ACIN1, CAPNS1, MAPK1, RRAS, LMNA, CAPN2, SPTAN1, CYCS, CDK1, PARP1, AIFM1, HSD17B10, UQCRH, ATP5D, PRDX5, ATP5L, UQCRB, MT-CO2, ATP5H, VDAC2, PDHA1, NDUFA5, SOD2, PARK7, GPD2, NDUFAB1, CYB5R3, NDUFB6, OGDH, ATP5F1, COX4I1,
AIFM1, SDHA, ATP5J, COX7A2, COX6B1, COX17, ATP5O, CPT1A, ATP5A1, VDAC3, NDUFS3, ATP5C1, FIS1, MT-ND1, PRDX3, NDUFB11, ATP5B, NDUFS8, UQCR10, CAT, UQCRC2, CYC1, COX5A, CYCS, VDAC1, UQCRC1, and COX5B. Notably, the proteomic analysis revealed a regulatory effect of PLB on apoptotic signaling pathways in PC-3 cells (Figure 11) but not in DU145 cells.

Moreover, Akt/mTOR signaling pathway plays a central role in the regulation of cell metabolism, growth, proliferation, and survival through the integration of both intracellular and extracellular signals. ${ }^{52}$ mTOR complex 1 and 2 are two distinct complexes in mTOR signaling pathway that transduce a variety of signals to downstream targets, including Akt, p70S6K, Atgs, eIF4G, PPAR- $\alpha$, and PPAR- $\gamma$, to modulate cell growth, cell proliferation, energy metabolism, and autophagy. ${ }^{52}$ Aberrant mTOR signaling pathway has been implicated in the pathogenesis of many diseases including cancer, and targeting mTOR signaling pathway 
Table 3 The top enriched clusters (Enrich score $>3$ ) by the DAVID database for the target list of PLB derived from molecular docking calculations

\begin{tabular}{|c|c|c|c|c|c|}
\hline Category & Term & Count & Fold enrichment & $P$-value & FDR \\
\hline Cluster I & Enrichment score: 7.89 & & & & \\
\hline GOTERM_BP_FAT & Response to organic substance & 22 & 5.16 & $5.44 \times 10^{-10}$ & $9.05 \times 10^{-9}$ \\
\hline GOTERM_BP_FAT & Response to endogenous stimulus & 17 & 7.10 & $1.19 \times 10^{-9}$ & $1.98 \times 10^{-8}$ \\
\hline GOTERM_BP_FAT & Response to hormone stimulus & 16 & 7.37 & $2.66 \times 10^{-9}$ & $4.42 \times 10^{-8}$ \\
\hline Cluster 2 & Enrichment score: 5.86 & & & & \\
\hline SP_PIR_KEYWORDS & Oxidoreductase & 16 & 6.60 & $1.31 \times 10^{-8}$ & $1.72 \times 10^{-7}$ \\
\hline GOTERM_BP_FAT & Oxidation reduction & 16 & 4.23 & $3.57 \times 10^{-6}$ & $5.93 \times 10^{-5}$ \\
\hline SP_PIR_KEYWORDS & NADP & 7 & 10.40 & $5.44 \times 10^{-5}$ & $7.13 \times 10^{-4}$ \\
\hline Cluster 3 & Enrichment score: 4.70 & & & & \\
\hline UP_SEQ_FEATURE & Active site: proton acceptor & 20 & 7.00 & $3.18 \times 10^{-11}$ & $4.25 \times 10^{-10}$ \\
\hline SP_PIR_KEYWORDS & Transferase & 27 & 4.49 & $5.82 \times 10^{-11}$ & $7.62 \times 10^{-10}$ \\
\hline SP_PIR_KEYWORDS & ATP & 13 & 12.77 & $3.42 \times 10^{-10}$ & $4.48 \times 10^{-9}$ \\
\hline Cluster 4 & Enrichment score: 3.9 I & & & & \\
\hline SP_PIR_KEYWORDS & NAD & 9 & 11.04 & $1.44 \times 10^{-6}$ & $1.88 \times 10^{-5}$ \\
\hline UP_SEQ_FEATURE & Nucleotide phosphate-binding region: NAD & 6 & 18.18 & $1.87 \times 10^{-5}$ & $2.50 \times 10^{-4}$ \\
\hline UP_SEQ_FEATURE & Binding site: NAD & 4 & 18.42 & $1.29 \times 10^{-3}$ & $1.71 \times 10^{-2}$ \\
\hline Cluster 5 & Enrichment score: 3.83 & & & & \\
\hline SMART & $\mathrm{ZnF}-\mathrm{C} 4$ & 11 & 54.28 & $2.21 \times 10^{-15}$ & $1.97 \times 10^{-14}$ \\
\hline UP_SEQ_FEATURE & DNA-binding region: nuclear receptor & 11 & 56.29 & $3.34 \times 10^{-15}$ & $4.45 \times 10^{-14}$ \\
\hline UP_SEQ_FEATURE & Zinc finger region: NR C4-type & II & 56.29 & $3.34 \times 10^{-15}$ & $4.45 \times 10^{-14}$ \\
\hline Cluster 6 & Enrichment score: 3.56 & & & & \\
\hline GOTERM_BP_FAT & Regulation of apoptosis & 18 & 3.79 & $2.98 \times 10^{-6}$ & $4.96 \times 10^{-5}$ \\
\hline GOTERM_BP_FAT & Regulation of programmed cell death & 18 & 3.75 & $3.41 \times 10^{-6}$ & $5.67 \times 10^{-5}$ \\
\hline GOTERM_BP_FAT & Regulation of cell death & 18 & 3.73 & $3.58 \times 10^{-6}$ & $5.96 \times 10^{-5}$ \\
\hline Cluster 7 & Enrichment score: 3.52 & & & & \\
\hline UP_SEQ_FEATURE & Binding site: substrate & 12 & 9.30 & $5.58 \times 10^{-8}$ & $7.46 \times 10^{-7}$ \\
\hline GOTERM_MF_FAT & $\begin{array}{l}\text { Steroid dehydrogenase activity, acting on the } \\
\mathrm{CH}-\mathrm{OH} \text { group of donors, NAD or NADP as } \\
\text { acceptor }\end{array}$ & 5 & 29.32 & $2.21 \times 10^{-5}$ & $2.99 \times 10^{-4}$ \\
\hline GOTERM_MF_FAT & Steroid dehydrogenase activity & 5 & 25.54 & $3.89 \times 10^{-5}$ & $5.26 \times 10^{-4}$ \\
\hline Cluster 8 & Enrichment score: $\mathbf{3 . 4 2}$ & & & & \\
\hline GOTERM_BP_FAT & Hexose metabolic process & 10 & 8.81 & $1.69 \times 10^{-6}$ & $2.82 \times 10^{-5}$ \\
\hline GOTERM_BP_FAT & Glucose metabolic process & 9 & 9.95 & $2.95 \times 10^{-6}$ & $4.90 \times 10^{-5}$ \\
\hline GOTERM_BP_FAT & Monosaccharide metabolic process & 10 & 7.62 & $5.58 \times 10^{-6}$ & $9.28 \times 10^{-5}$ \\
\hline Cluster 9 & Enrichment score: 3.35 & & & & \\
\hline GOTERM_MF_FAT & Identical protein binding & 14 & 3.46 & $1.53 \times 10^{-4}$ & $2.06 \times 10^{-3}$ \\
\hline GOTERM_MF_FAT & Protein dimerization activity & 12 & 3.51 & $5.19 \times 10^{-4}$ & $6.99 \times 10^{-3}$ \\
\hline GOTERM_MF_FAT & Protein homodimerization activity & 9 & 4.27 & $1.10 \times 10^{-3}$ & $1.49 \times 10^{-2}$ \\
\hline Cluster 10 & Enrichment score: 3.26 & & & & \\
\hline GOTERM_MF_FAT & Vitamin binding & 7 & 8.53 & $1.58 \times 10^{-4}$ & $2.13 \times 10^{-3}$ \\
\hline GOTERM_MF_FAT & Retinoid binding & 4 & 30.16 & $2.87 \times 10^{-4}$ & $3.87 \times 10^{-3}$ \\
\hline GOTERM_BP_FAT & Diterpenoid metabolic process & 4 & 29.41 & $3.12 \times 10^{-4}$ & $5.18 \times 10^{-3}$ \\
\hline
\end{tabular}

Notes: Clusters were sorted by the enrichment score. Only the top three terms in each cluster were listed.

Abbreviations: FDR, false discovery rate; NAD, nicotineamide adenine dinucleotide; NADP, nicotinamide adenine dinucleotide phosphate; PLB, plumbagin.

may be a promising strategy for cancer therapy..$^{53}$ As showed in Figures 12 and 13, PLB exhibited a capability of modulating mTOR signaling pathway in both cell lines. The results showed that PLB decreased the expression of FKBP1, Rho, Rac, eIF3, eIF4B, and eIF4G, but increased the expression of Erk1/2, Ras, PP2, and eIF4A in PC-3 cells (Figure 12), whereas there were less targets regulated by PLB in DU145 cells, ie, only FKBP1, eIF4A, and 40S ribosome (Figure 13). Taken together, the results suggest that the regulatory effects of PLB on apoptosis, mitochondrial function, and mTOR signaling pathway contribute to the cancer cell killing of PLB in PC-3 and DU145 cells.

\section{PLB regulates EMT pathways in PC- 3 cells}

EMT has a close association with cell migration and invasion and it plays an important role in cancer metastasis. ${ }^{21}$ Suppressing the progress of EMT will be clinically helpful for cancer therapy. We analyzed the effect of PLB on 
Table 4 The top enriched KEGG pathways (FDR $<0.1$ ) by the DAVID database for the target list of PLB derived from molecular docking calculations

\begin{tabular}{|c|c|c|c|c|}
\hline Pathway & Gene count & Fold enrichment & $P$-value & FDR \\
\hline Metabolism of xenobiotics by cytochrome P450 & 6 & 7.48 & 0.0011 & 0.012 \\
\hline Progesterone-mediated oocyte maturation & 7 & 6.09 & $8.58 \times 10^{-4}$ & 0.010 \\
\hline ErbB signaling pathway & 7 & 6.02 & $9.12 \times 10^{-4}$ & 0.010 \\
\hline VEGF signaling pathway & 6 & 5.98 & 0.0029 & 0.033 \\
\hline Fc epsilon RI signaling pathway & 6 & 5.75 & 0.0034 & 0.039 \\
\hline Neurotrophin signaling pathway & 9 & 5.43 & $1.95 \times 10^{-4}$ & 0.002 \\
\hline Colorectal cancer & 6 & 5.34 & 0.0047 & 0.053 \\
\hline Prostate cancer & 6 & 5.04 & 0.0060 & 0.068 \\
\hline Insulin signaling pathway & 7 & 3.88 & 0.0083 & 0.092 \\
\hline MAPK signaling pathway & 10 & 2.80 & 0.0078 & 0.086 \\
\hline
\end{tabular}

Note: Clusters were sorted by the enrichment fold.

Abbreviations: FDR, false discovery rate; KEGG, Kyoto Encyclopedia of Genes and Genomes; PLB, plumbagin.

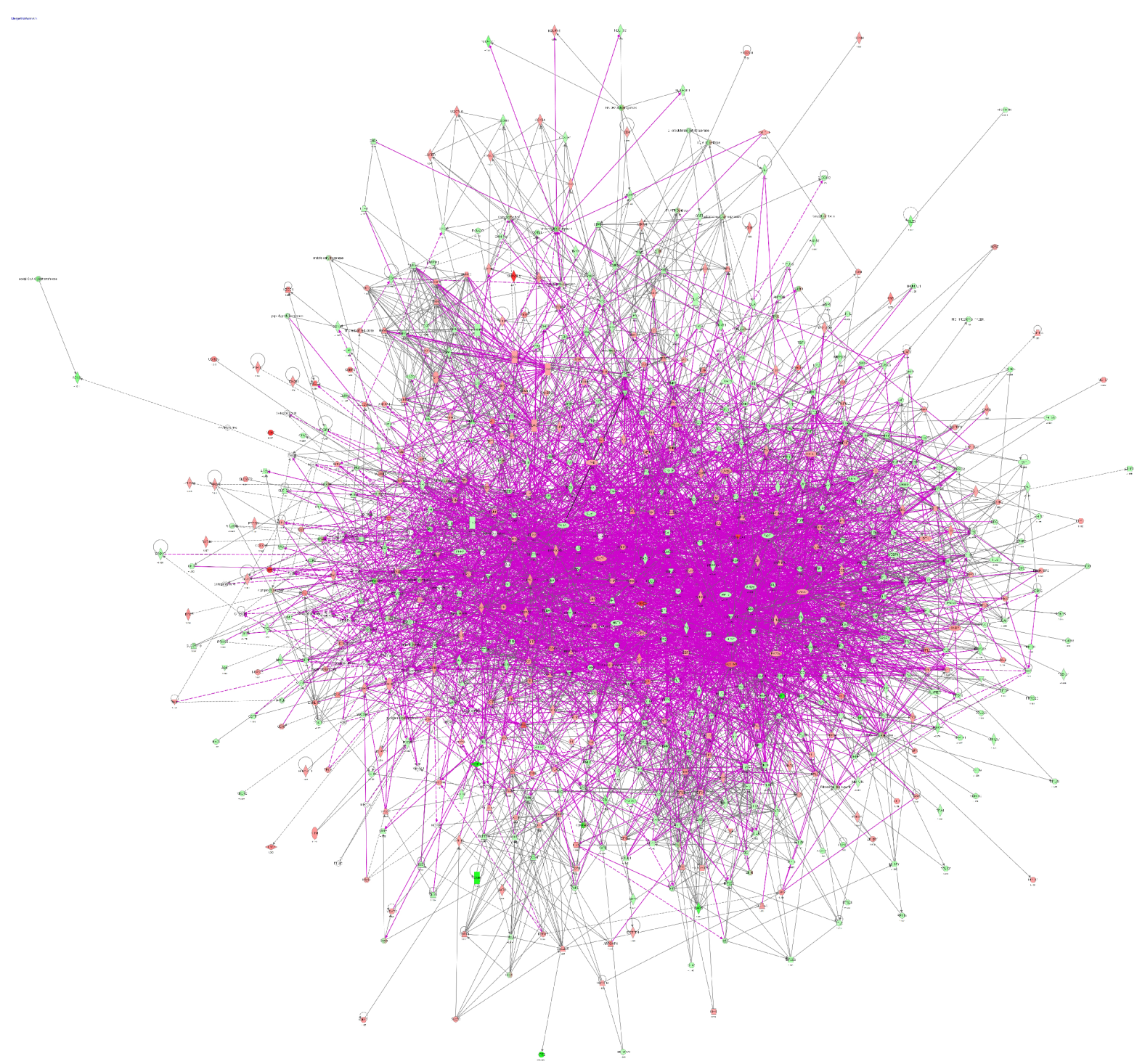

Figure 6 Proteomic analysis revealed molecular interactome regulated by PLB in PC-3 cells.

Notes: PC-3 cells were treated with $5 \mu$ M PLB for 24 hours and the protein samples were subject to quantitative proteomic analysis. There were I,225 molecules and 34 I related pathways regulated by PLB in PC-3 cells. Red indicates an upregulation; green indicates a downregulation; brown indicates a predicted activation; and blue indicates a predicted inhibition. The intensity of green and red molecule colors indicates the degree of down- or upregulation, respectively. Solid arrows indicate direct interaction and dashed arrows indicate indirect interaction.

Abbreviation: PLB, plumbagin. 


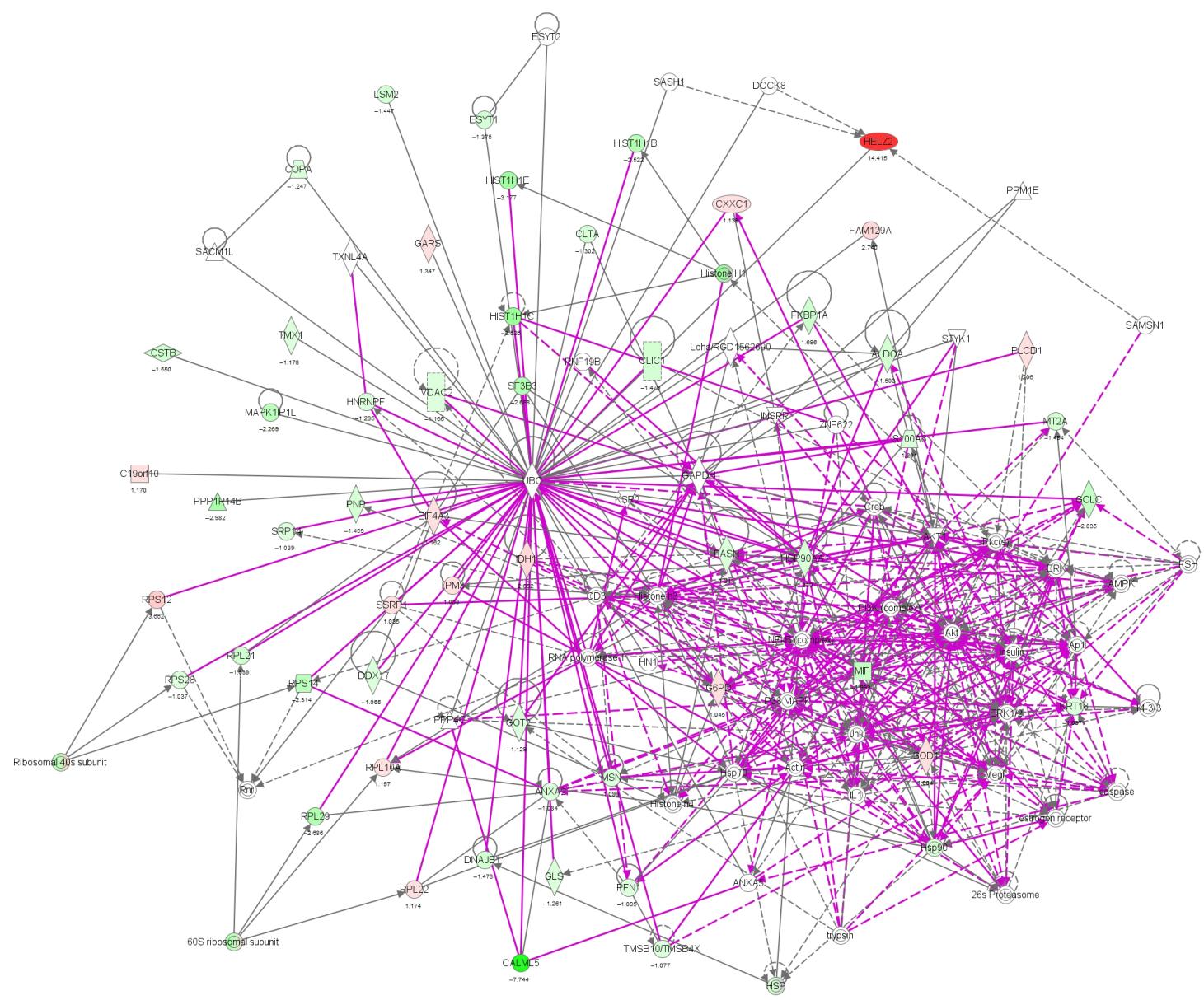

Figure 7 Proteomic analysis revealed molecular interactome regulated by PLB in DUI45 cells.

Notes: DUI 45 cells were treated with $5 \mu$ M PLB for 24 hours and the protein samples were subject to quantitative proteomic analysis. There were 267 molecules and I07 related pathways regulated by PLB in DUI 45 cells. Red indicates an upregulation; green indicates a downregulation; brown indicates a predicted activation; and blue indicates a predicted inhibition. The intensity of green and red molecule colors indicates the degree of down- or upregulation, respectively. Solid arrows indicate direct interaction and dashed arrows indicate indirect interaction.

Abbreviation: PLB, plumbagin.

EMT-related proteins and signaling pathways using SILACbased proteomic approach. The proteomic data showed that PLB regulated epithelial adherent junction signaling pathway in PC-3 cells involving a number of functional proteins. These included RAP1B, MYL6, ARPC1B, ACTA2, IQGAP1, TUBB, CDC42, ACTR3, ARPC3, TUBA1C, VCL, CTNNB1, ACTN1, ACTR2, TUBB3, LMO7, TUBB4B, RRAS, TUBB2A, TUBA4A, RAC1, ACTG1, TUBA1B, TUBA1A, MYH9, ZYX, ACTN4, and ARPC4 (Table 5; Figure 14); whereas the proteomic analysis did not show remarkable regulatory effect of PLB on EMT-associated proteins and signaling pathways in DU145 cells.

\section{PLB regulates Sirt I-mediated pathways in PC-3 and DUI 45 cells}

The Sirt family of proteins (Sirt1-7) encode a group of evolutionarily conserved, class III, and $\mathrm{NAD}^{+}$-dependent histone deacetylases involving many critical cellular processes, including cell cycle regulation, cell differentiation, genomic stability, tumorigenesis, oxidative stress response, aging, and energy metabolism through PPAR-, p53-, nuclear factor- $\kappa B$ (NF-KB)-, AMPK-, and mTOR-mediated signaling pathways. ${ }^{54}$ The proteomic data showed that PLB regulated NAD biosynthesis, phosphorylation, and dephosphorylation with the involvement of ACP1 and nicotinamide phosphoribosyltransferase (NAMPT) in PC-3 cells (Table 5). NAMPT, also known as pre-B-cell colony-enhancing factor 1 or visfatin, is a rate-limiting step in the $\mathrm{NAD}^{+}$biosynthesis salvage pathway, and $\mathrm{NAD}^{+}$is an essential substrate for Sirt $1 .{ }^{55}$ Moreover, PLB treatment regulated the $\mathrm{p} 53$ signaling pathway with the involvement of PRKDC, PCNA, GNL3, SERPINB5, SFN, ST13, and CTNNB1, and modulated NF- $\mathrm{KB}$ signaling pathway with the involvement of ITGB1, MAPK1, RRAS, ITGA2, and ITGA6 in PC-3 cells (Table 5). Notably, PLB treatment regulated PPAR signaling pathway in both PC-3 and DU145 cells involving a number of protein molecules, 


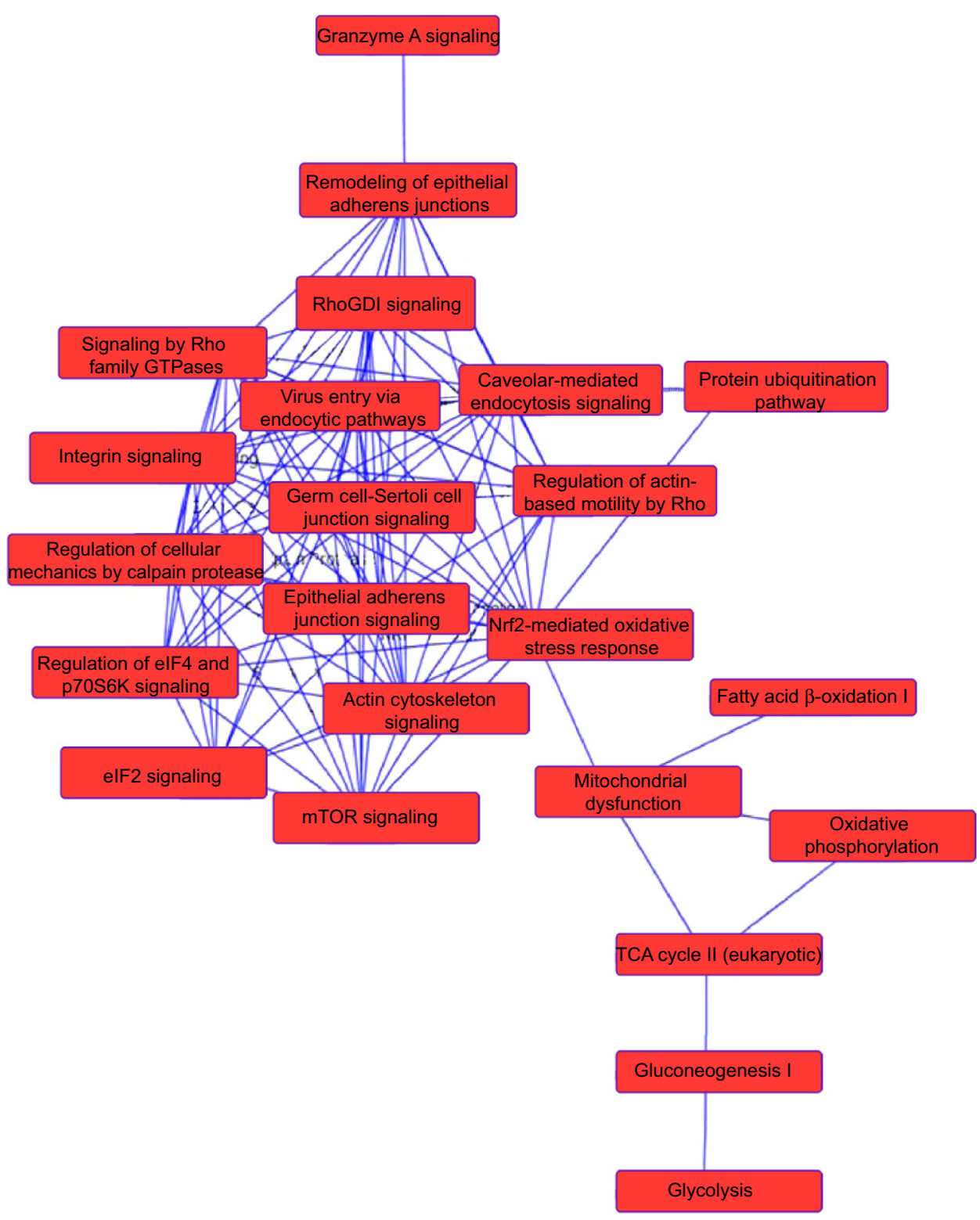

Figure 8 Proteomic analysis revealed a network of signaling pathways regulated by PLB in PC-3 cells.

Notes: A network of signaling pathways was analyzed by IPA according to the I,225 molecules and $34 \mathrm{I}$ related pathways which were regulated by PLB in PC-3 cells. Abbreviations: IPA, Ingenuity Pathway Analysis; PLB, plumbagin; TCA, tricarboxylic acid cycle.

such as HSP90B1, IL18, MAPK1, HSP90AB1, RRAS, and HSP90AA1(Tables 5 and 6). Taken together, the proteomic data suggest that PLB may exhibit a regulatory effect on Sirt1mediated signaling pathways in both PC-3 and DU145 cells.

\section{PLB regulates redox homeostasis involving ROS-} and Nrf2-mediated signaling pathways in both PC-3 and DUI 45 cells

Our previous study has shown that induction of ROS generation and modulation of related signaling pathways contribute to the anticancer effects of PLB. ${ }^{30}$ In this study, we observed that PLB regulated several critical signaling pathways related to ROS generation and redox homeostasis in PC-3 and DU145 cells. Our quantitative proteomic study showed that PLB treatment regulated oxidative phosphorylation, nuclear factor erythroid 2-related factor 2 (Nrf2)-mediated oxidative stress response (Figures 15 and 16), and superoxide radical degradation in PC-3 and DU145 cells (Tables 5 and 6). A number of functional proteins - SOD1/2, GSTK1, GSTP1, MGST1, HSD17B10, DHRS9, AKR1A1, ADH5, ESD, ALDH1A3, 1L1, 3A2, 9A1 - were found to be involved in these pathways as well as $18 \mathrm{~A} 1$, NQO1, and mitochondria complexes. Notably, Nrf2-mediated signaling pathway plays a critical role in the maintenance of intracellular redox 


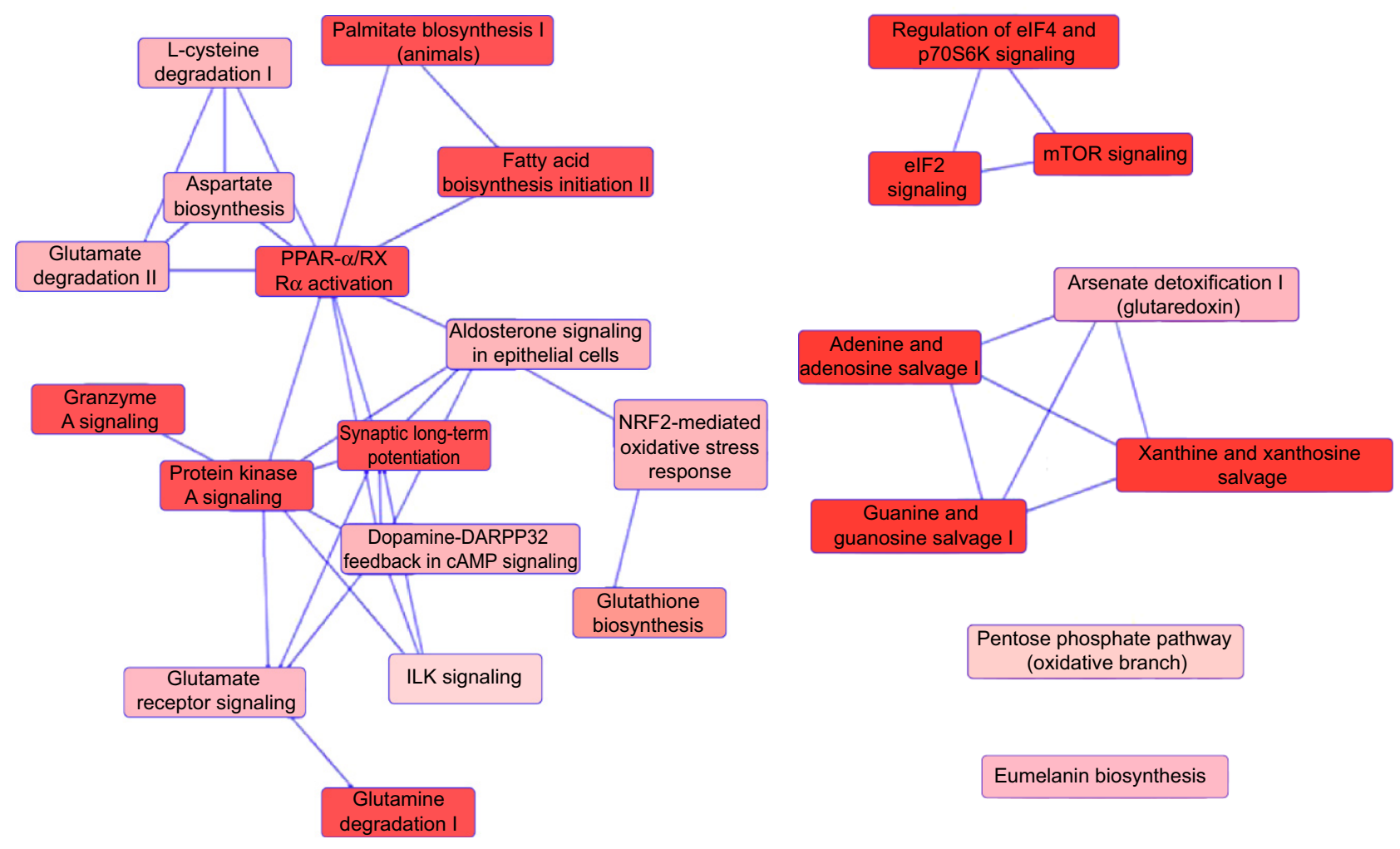

Figure 9 Proteomic analysis revealed networks of signaling pathways regulated by PLB in DUI45 cells.

Notes: Networks of signaling pathways were analyzed by IPA according to 267 molecules and I 07 related pathways which were regulated by PLB in DUI 45 cells.

Abbreviations: cAMP, cyclic adenosine monophosphate; IPA, Ingenuity Pathway Analysis; PLB, plumbagin.

homeostasis in response to various stimuli via regulating antioxidant responsive elements in the target genes..$^{56,57}$ The proteomic data indicate that modulation of the expression of functional proteins involved in Nrf2-mediated signaling pathway may be an important contributor to the anticancer effect of PLB.

\section{Differential responses to PLB treatment in PC-3 and DUI 45 cells}

There were substantial differences in the response to PLB treatment between PC-3 and DU145 cells. In PC-3 cells, the PLBregulated network of signaling pathways included granzyme A signaling pathway, remodeling of epithelial adherent junctions, Rho signaling pathway, endocytosis signaling pathway, integrin signaling pathway, protein ubiquitination signaling pathway, EIF4/p70 S6K signaling pathway, Nrf2-mediated signaling pathway, EIF2 signaling pathway, mTOR signaling pathway, mitochondrial dysfunction, fatty acid $\beta$-oxidation, tricarboxylic acid cycle, and glycolysis (Figure 8). These signaling pathways played a critical role in the regulation of cell proliferation, migration, and programmed cell death. In DU145 cells, different network of signaling pathways in response to the PLB treatment was observed. These mainly included palmitate biosynthesis, fatty acid biosynthesis, aspirate biosynthesis, L-cysteine degradation, glutamate degradation, PPAR- $\alpha / R X R \alpha$ activation, protein kinase A signaling pathway, granzyme A signaling pathway, glutamate receptor signaling pathway, Nrf2-mediated signaling pathway, EIF2 signaling pathway, mTOR signaling pathway, and EIF4/p70 S6K signaling pathway. These pathways played important roles in the regulation of cell and energy metabolism, cell growth, cell survival, and programmed cell death.

Moreover, the proteomic data showed differences in the top five signaling pathways in response to PLB treatment in both cell lines (Tables 7 and 8). In PC-3 cells, the top five signaling pathways were EIF2 signaling pathway, EIF4/p70 S6K signaling pathway, mTOR signaling pathway, protein ubiquitination signaling pathway, and mitochondrial dysfunction signaling pathway (Table 7). In DU145 cells, the top five signaling pathways were EIF2 signaling pathway, granzyme A signaling pathway, PPAR- $\alpha / R X R \alpha$ signaling pathway, mTOR signaling pathway, and protein kinase A signaling pathway (Table 8). mTOR signaling pathway was regulated by PLB in both cell lines, indicating that it may play 


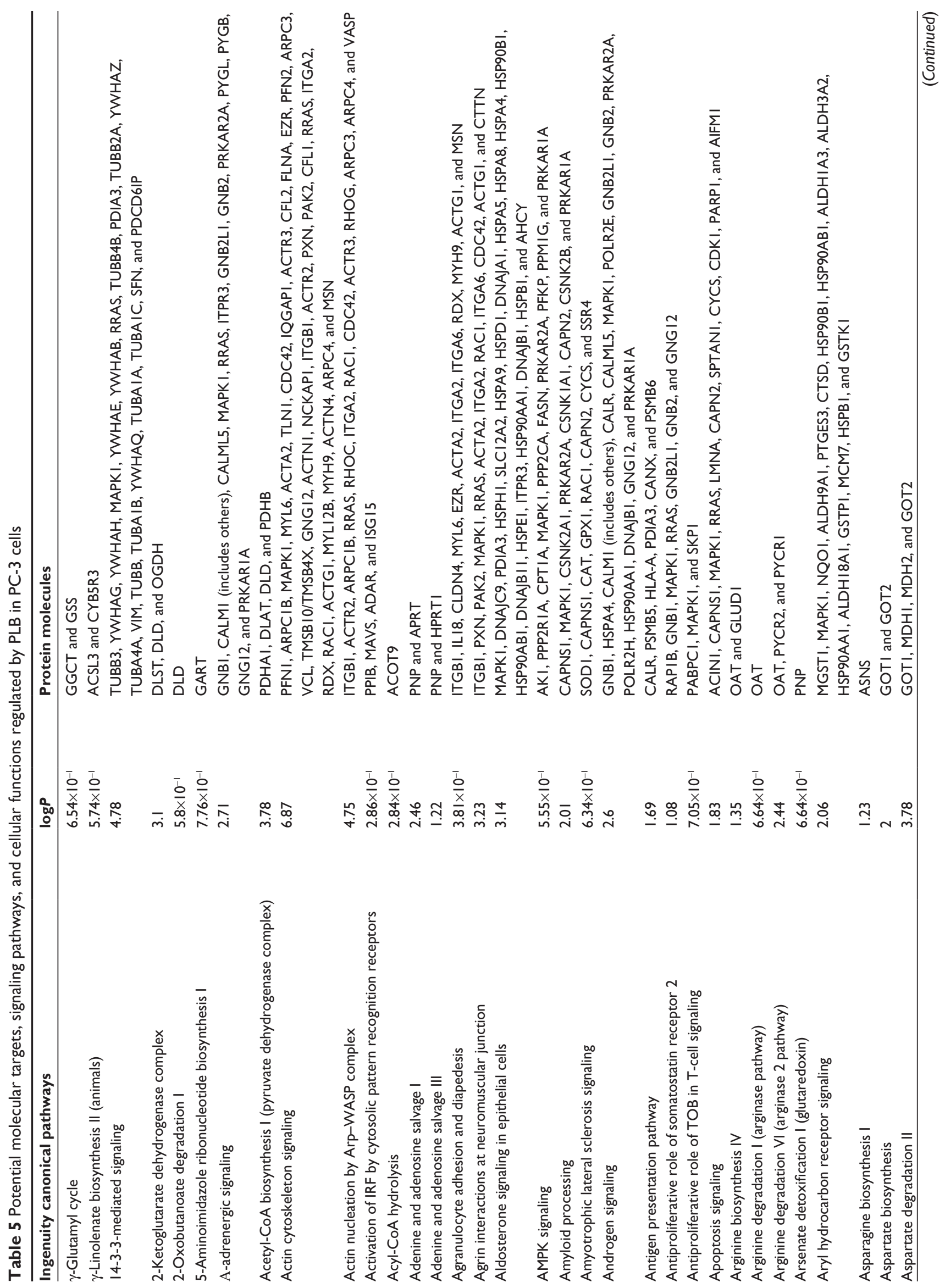




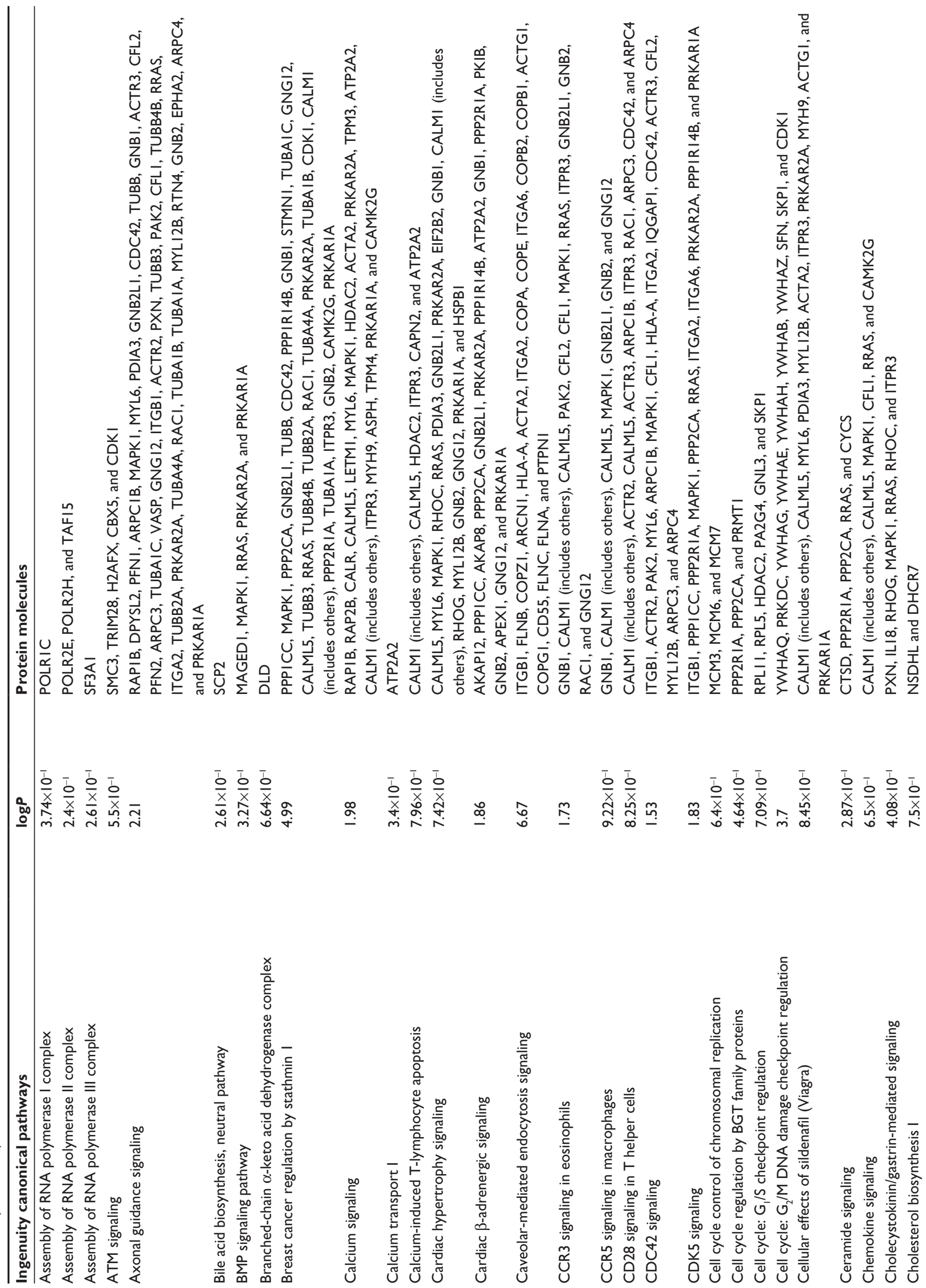



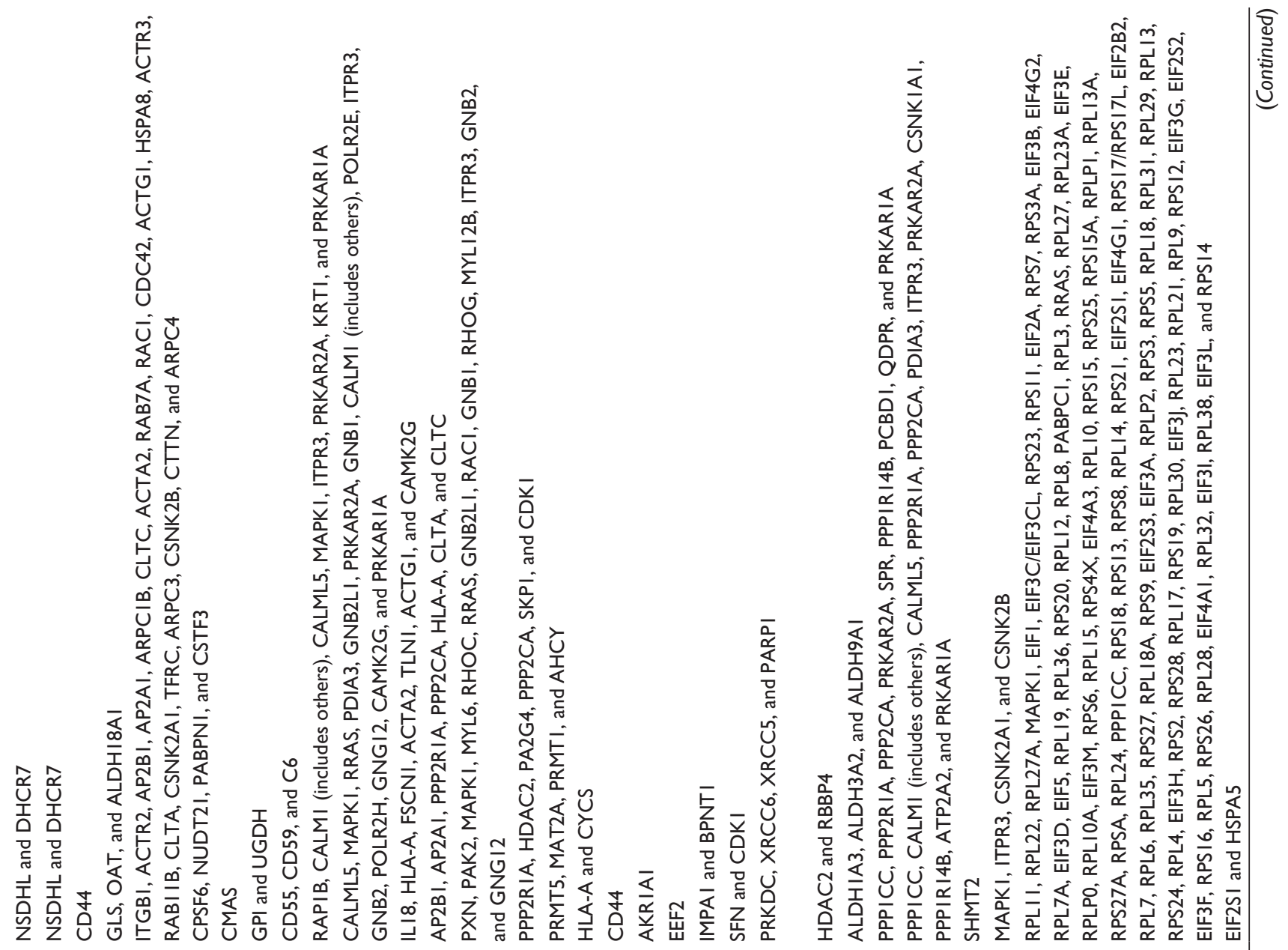

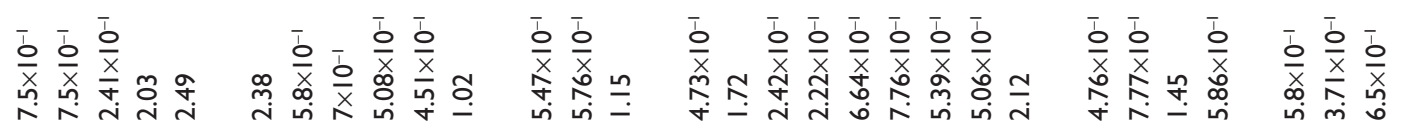

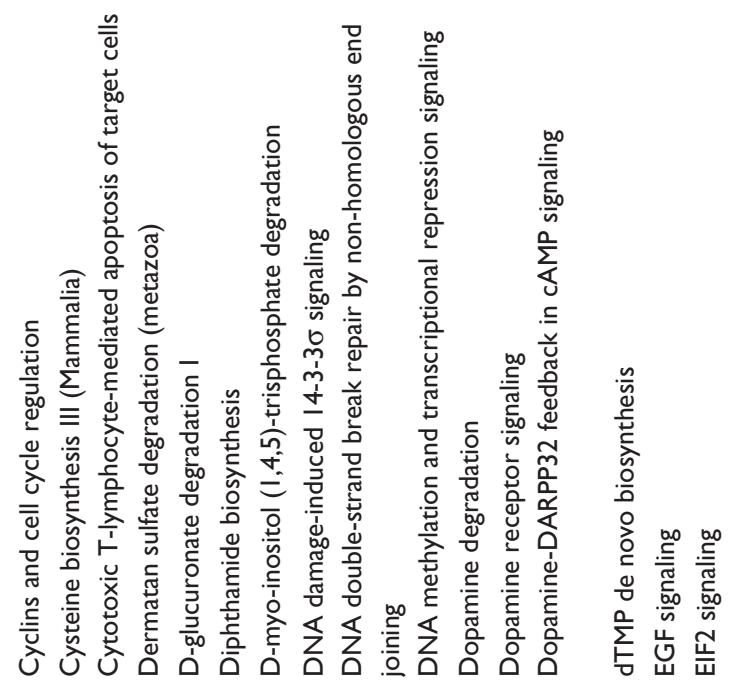




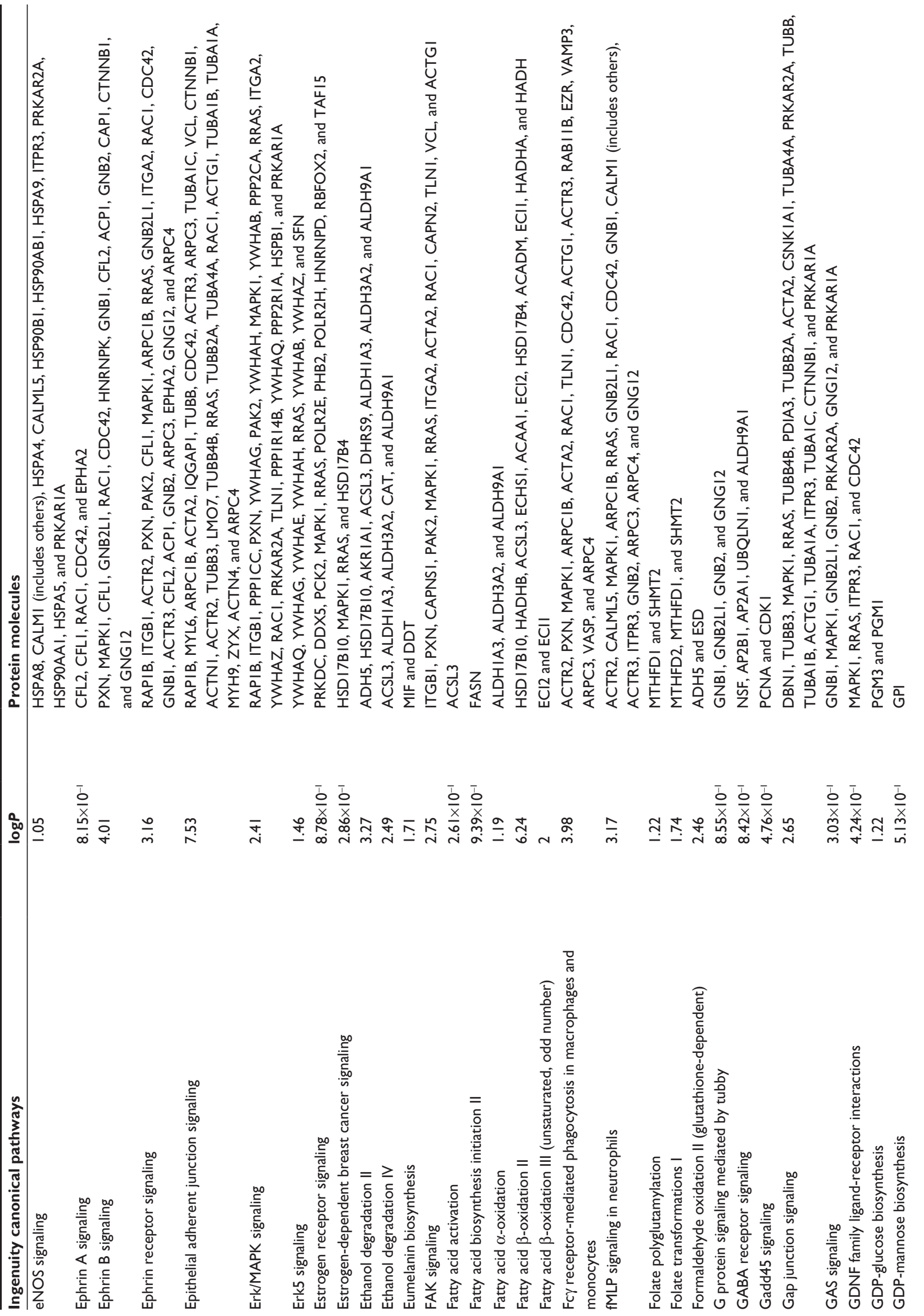




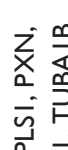 \\ 产它 \\ $\bar{z} \bar{v}$}

它

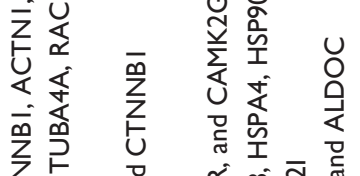

Z U v क

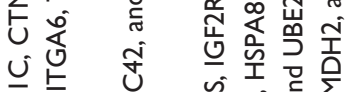

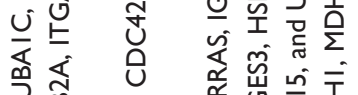

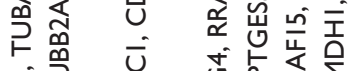

Uंग प

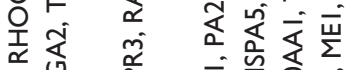

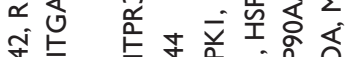

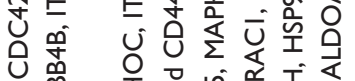

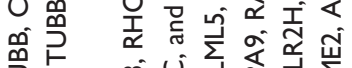

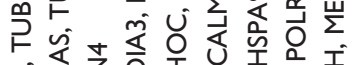

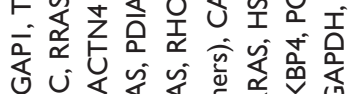

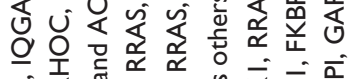

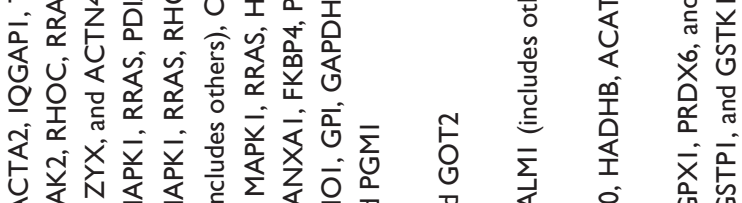

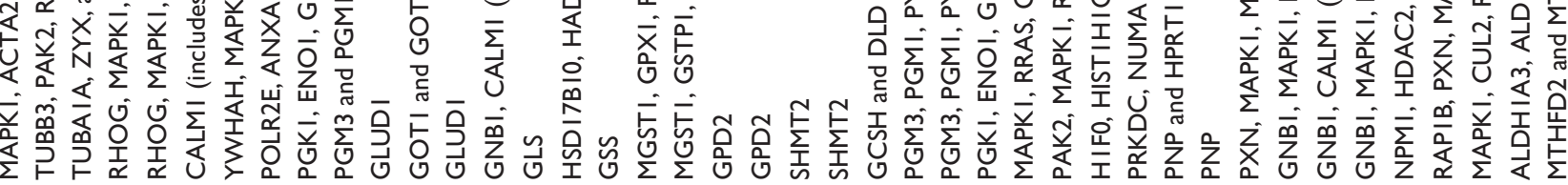

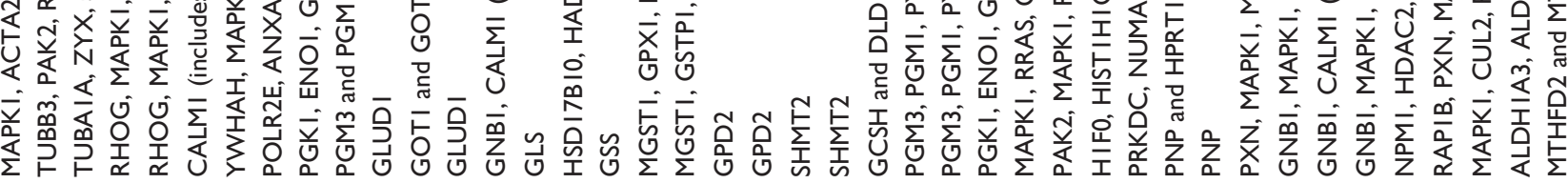

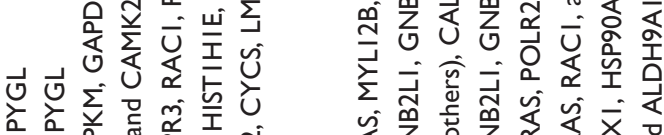

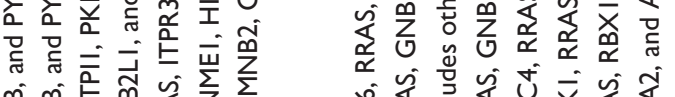
की

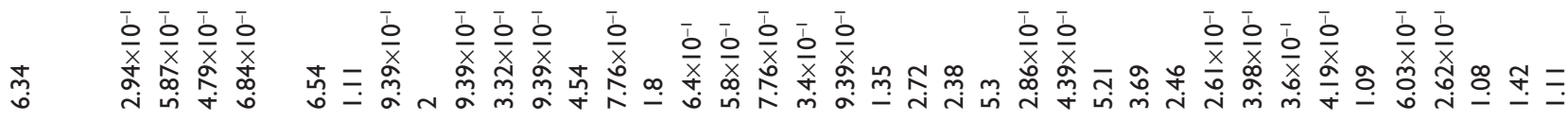

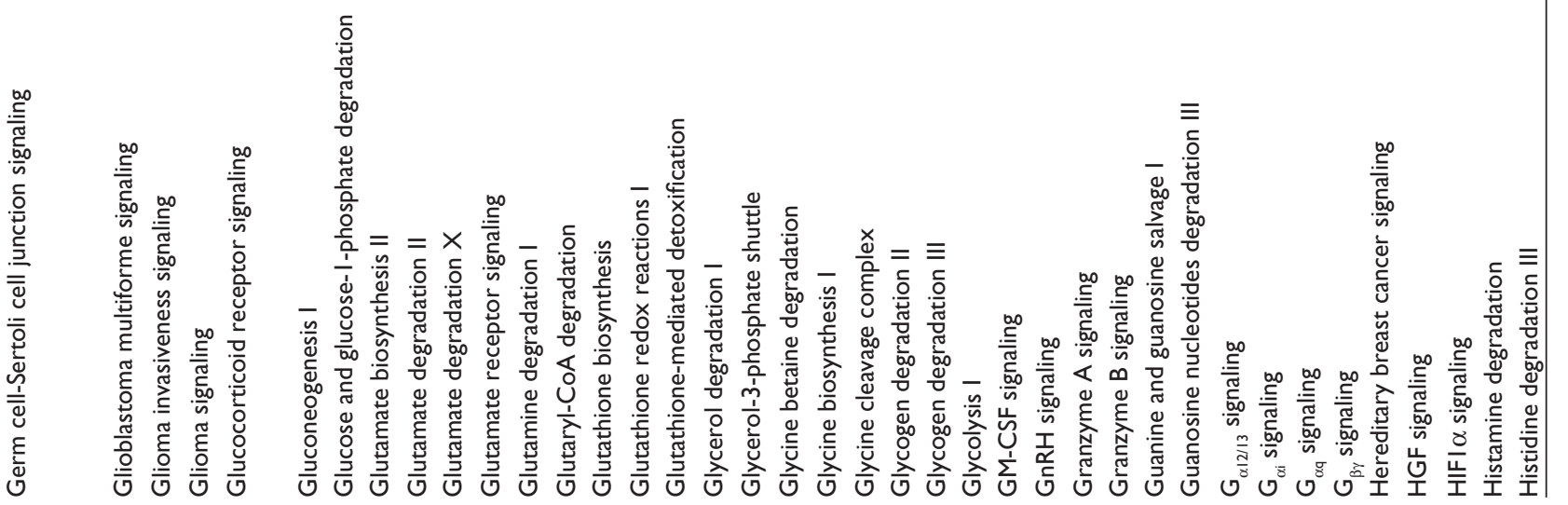




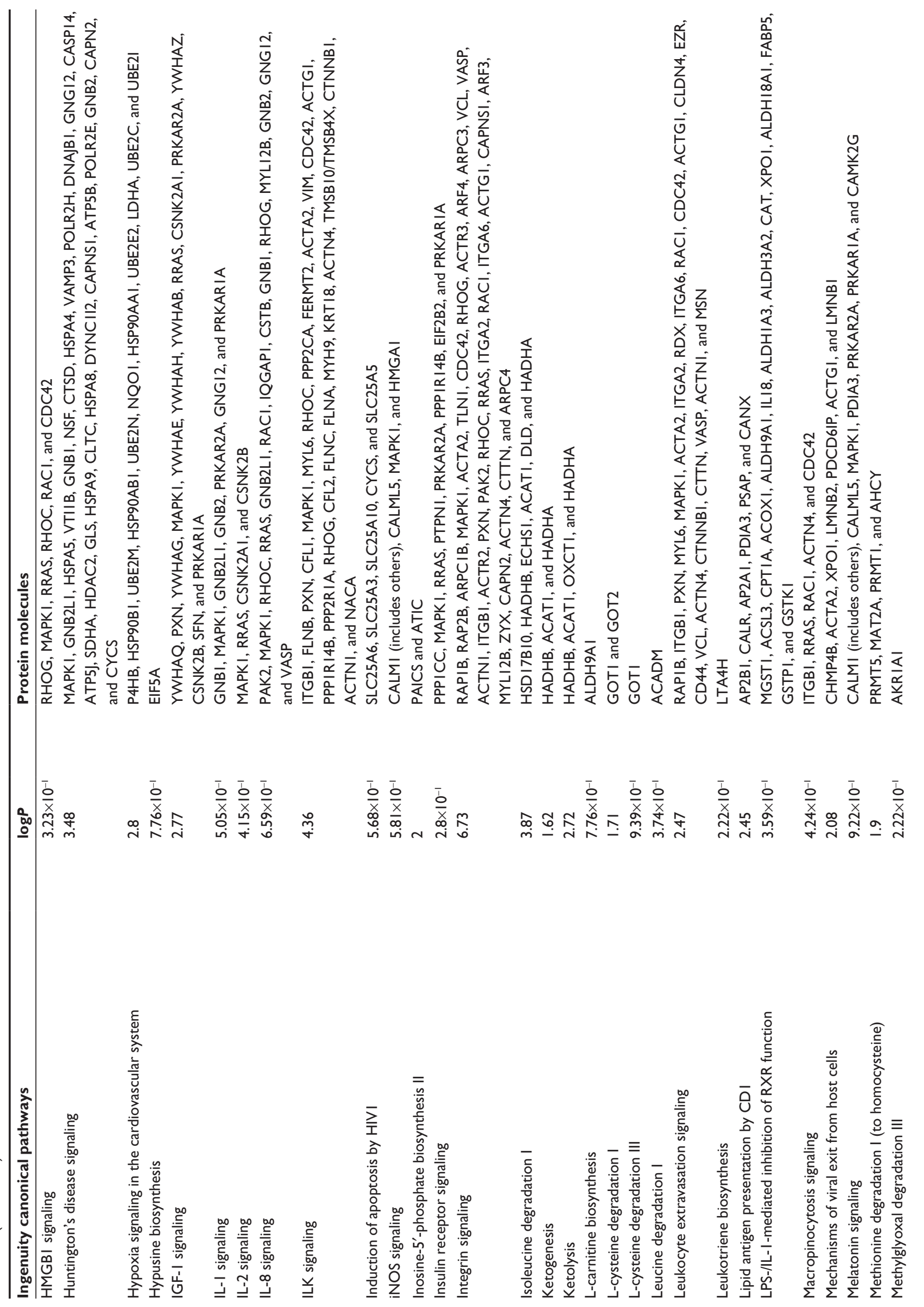




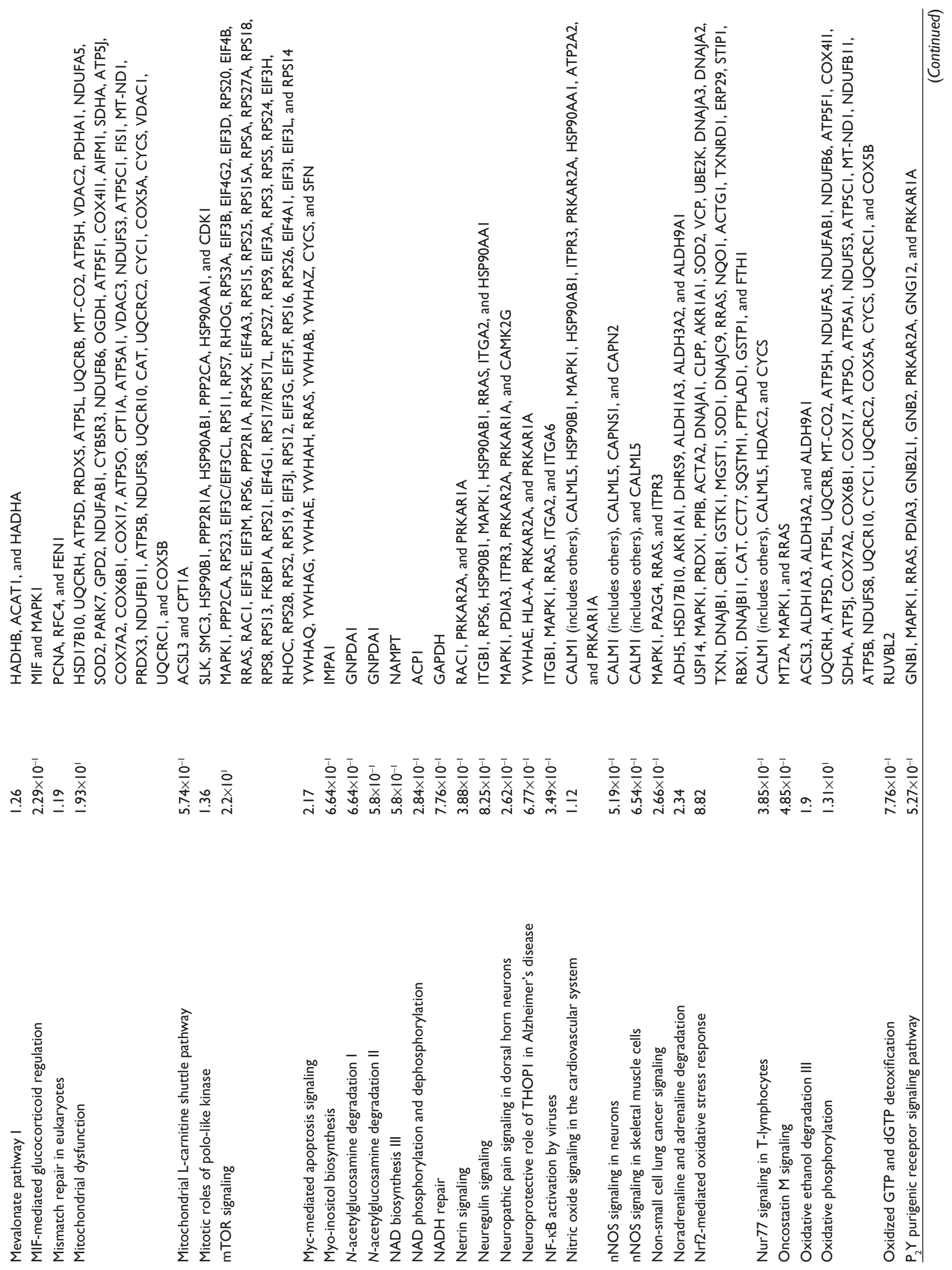




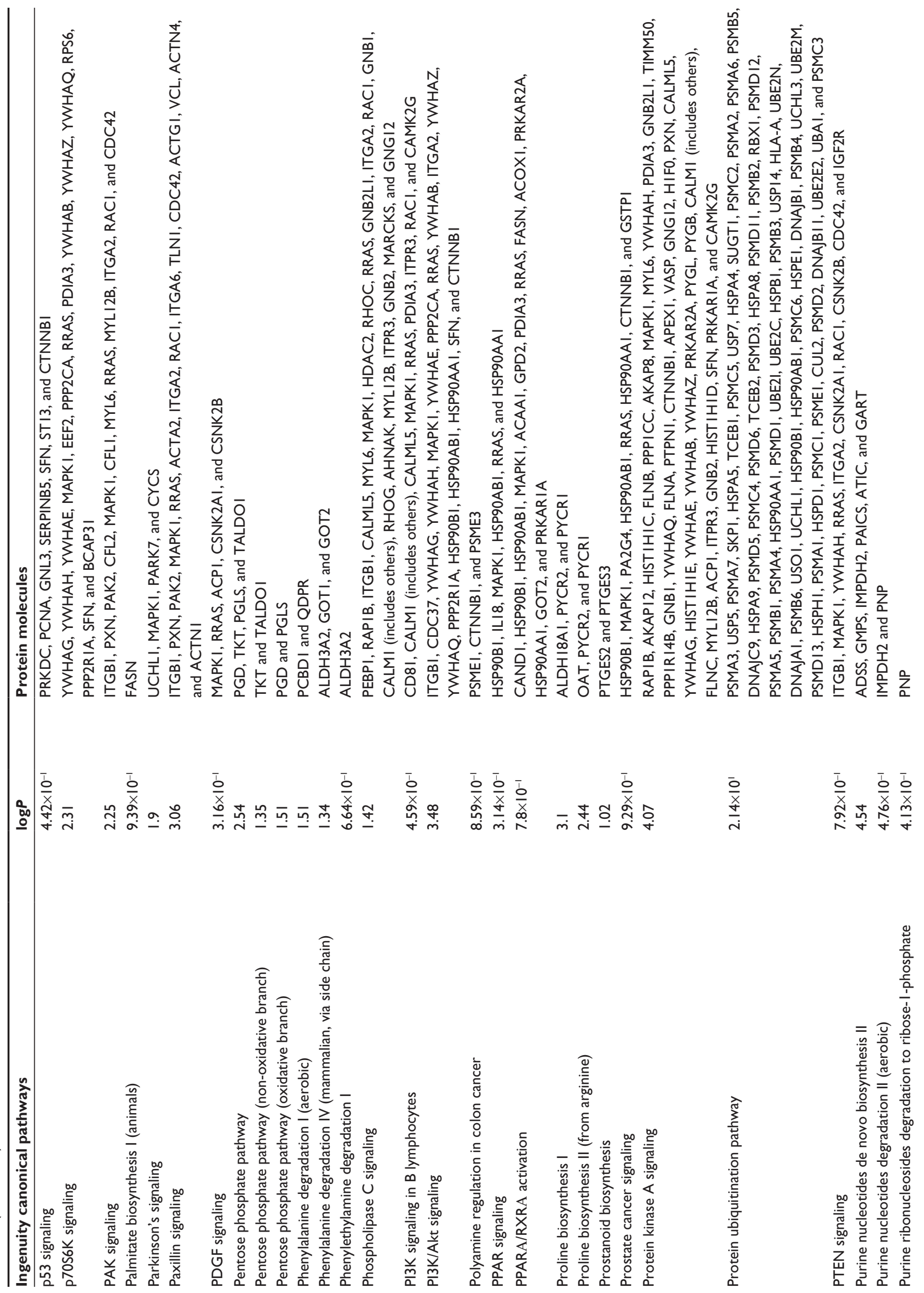



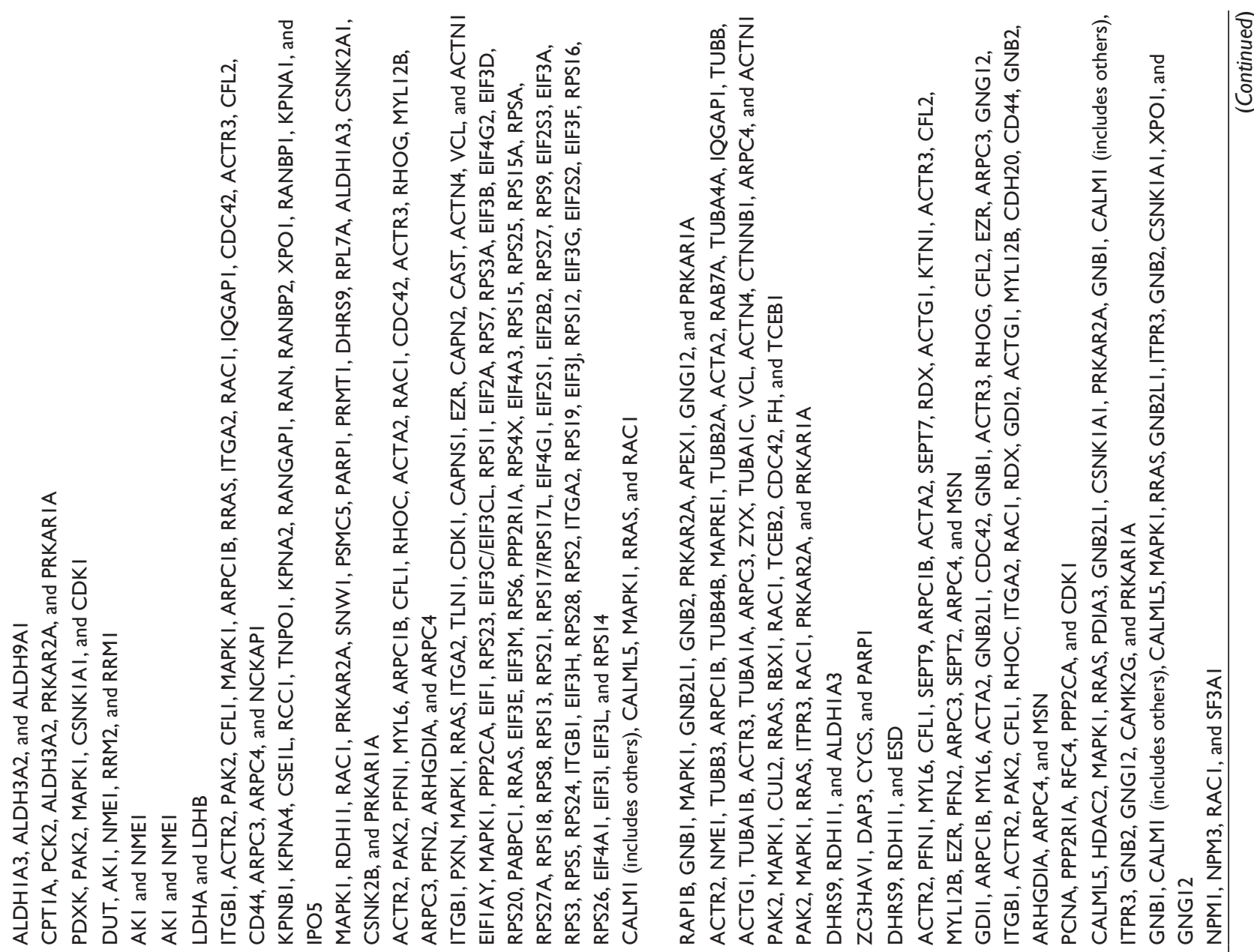

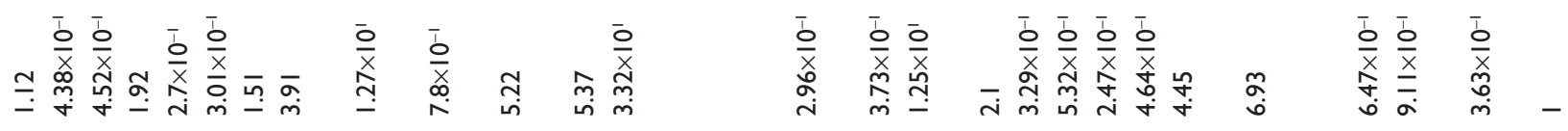
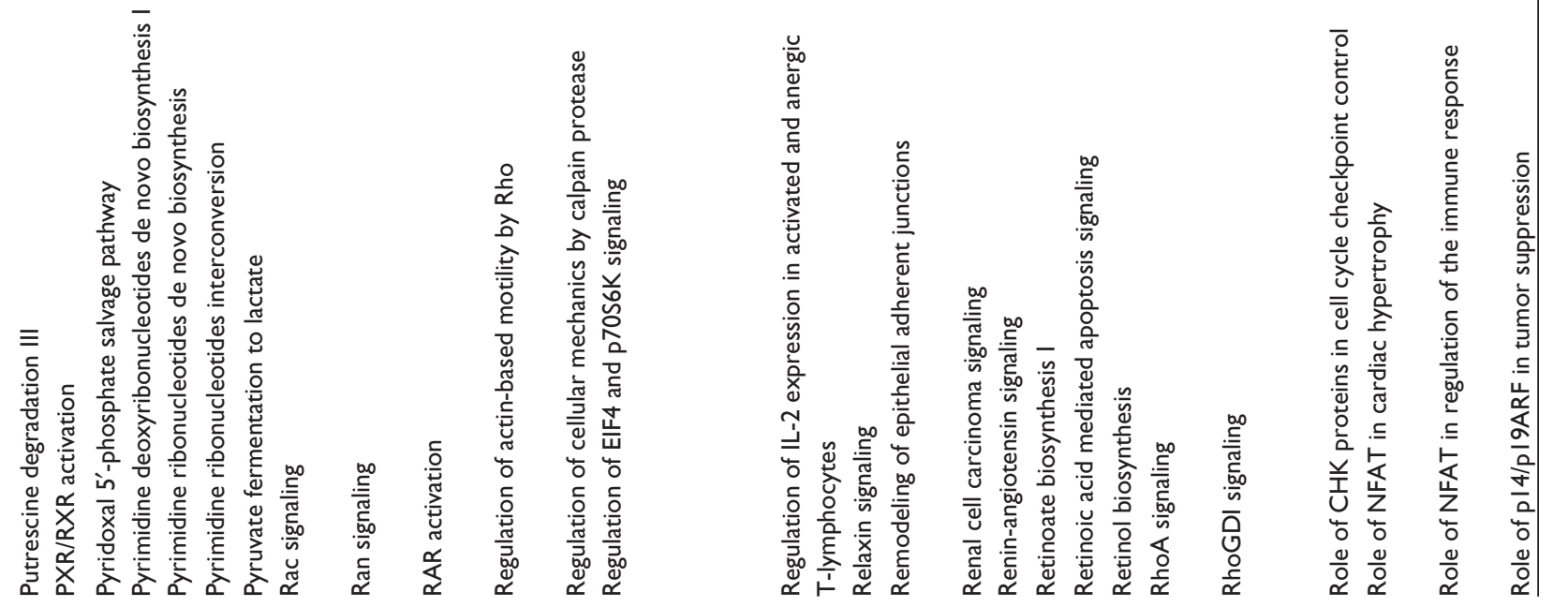


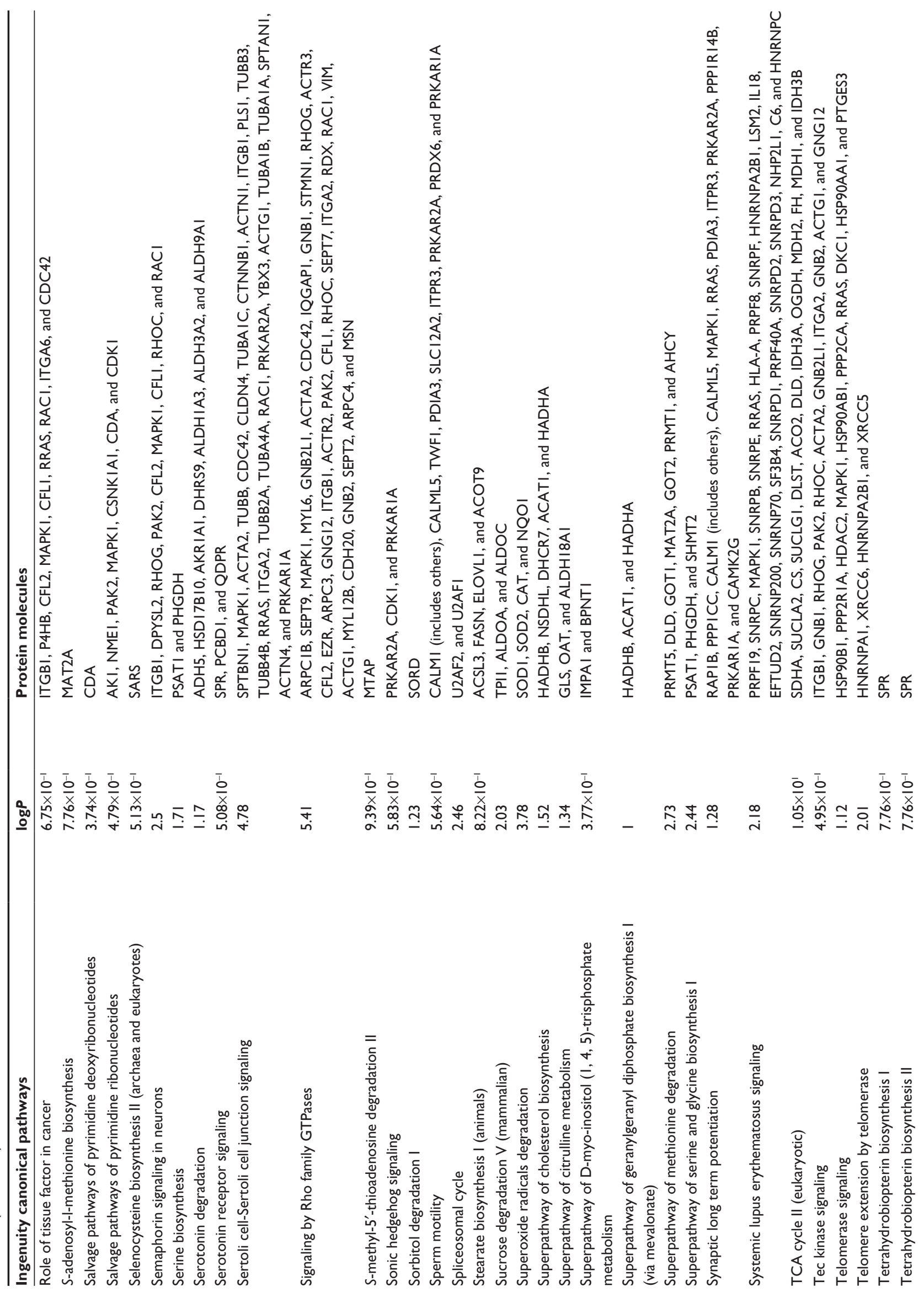




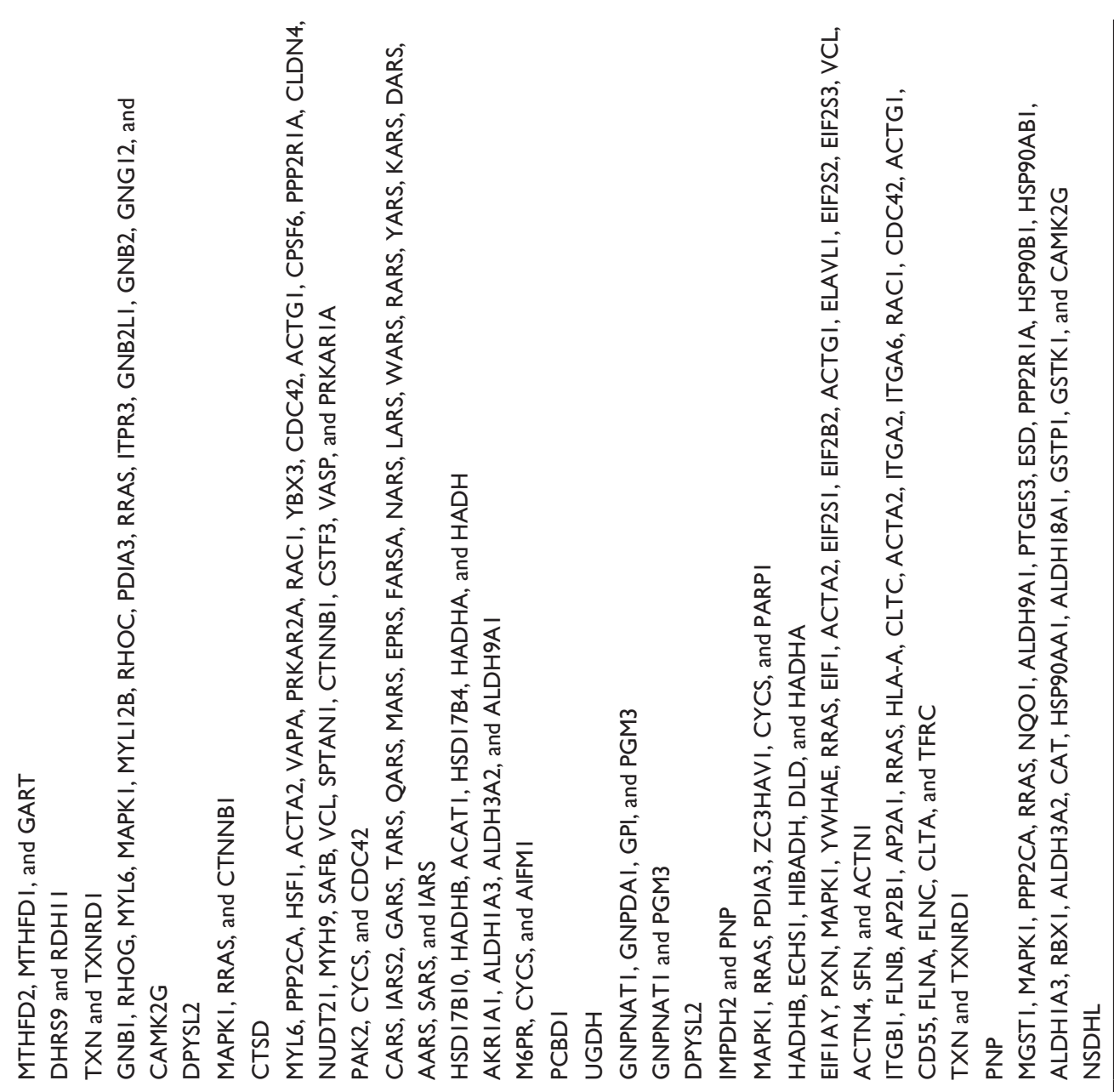

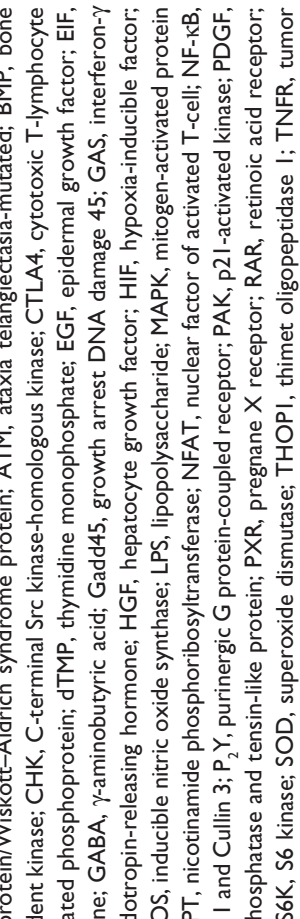

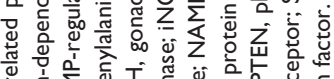

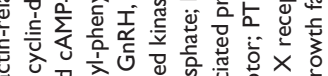

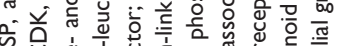

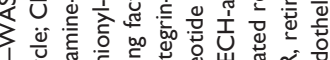

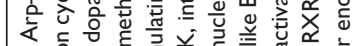

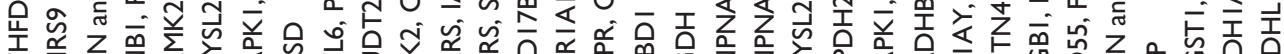

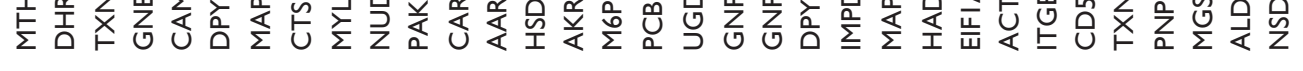

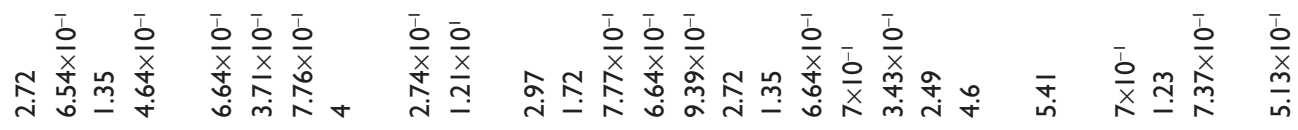
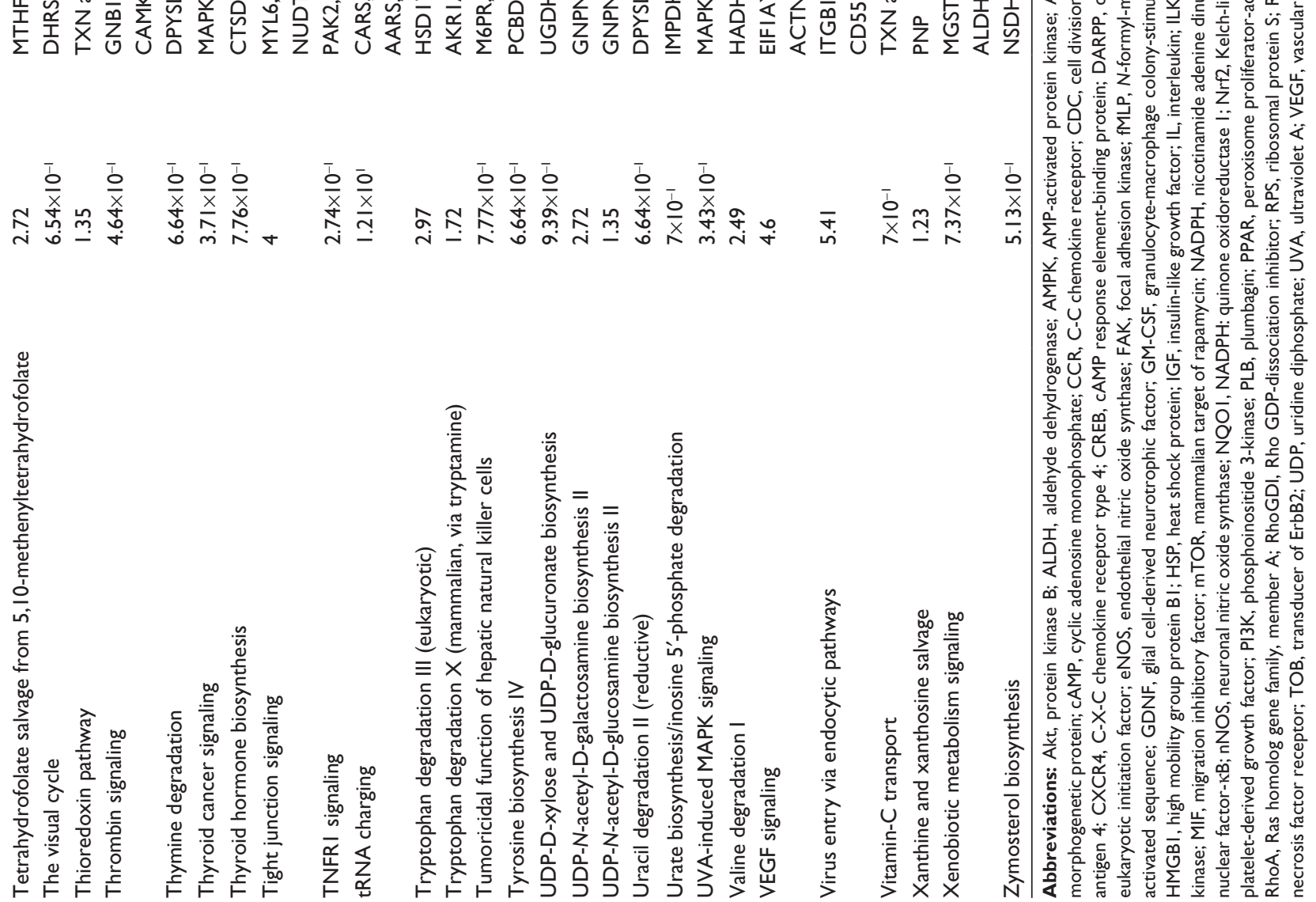
Table 6 Potential molecular targets, signaling pathways, and cellular functions regulated by PLB in DUI45 cells

\begin{tabular}{|c|c|c|}
\hline Ingenuity canonical pathways & $\log P$ & Protein molecules \\
\hline$\gamma$-Glutamyl cycle & 1.41 & GCLC \\
\hline |4-3-3-mediated signaling & $5.5 \times 10^{-1}$ & PLCDI \\
\hline 3-Phosphoinositide biosynthesis & $4.52 \times 10^{-1}$ & PPPIRI4B \\
\hline 3-Phosphoinositide degradation & $4.78 \times 10^{-1}$ & PPPIRI4B \\
\hline Actin cytoskeleton signaling & 1.63 & PFNI, TMSBI0/TMSB4X, and MSN \\
\hline Adenine and adenosine salvage I & 2.25 & PNP \\
\hline Adenine and adenosine salvage III & 1.71 & PNP \\
\hline Adenosine nucleotides degradation II & 1.33 & PNP \\
\hline Agranulocyte adhesion and diapedesis & $3.82 \times 10^{-1}$ & MSN \\
\hline Aldosterone signaling in epithelial cells & 2.04 & PLCDI, DNAJBII, and HSP90AAI \\
\hline AMPK signaling & $4.98 \times 10^{-1}$ & FASN \\
\hline Amyotrophic lateral sclerosis signaling & $6.16 \times 10^{-1}$ & SODI \\
\hline Androgen signaling & $\mathrm{I} .4 \mathrm{I}$ & CALML5 and HSP90AAI \\
\hline Antioxidant action of vitamin $C$ & $6.2 \times 10^{-1}$ & PLCDI \\
\hline Arsenate detoxification I (glutaredoxin) & 1.95 & PNP \\
\hline Aryl hydrocarbon receptor signaling & $4.85 \times 10^{-1}$ & HSP90AAI \\
\hline Aspartate biosynthesis & 2.07 & GOT2 \\
\hline Aspartate degradation II & 1.71 & GOT2 \\
\hline Axonal guidance signaling & $4.63 \times 10^{-1}$ & PLCDI and PFNI \\
\hline B-cell receptor signaling & $4.06 \times 10^{-1}$ & CALML5 \\
\hline Breast cancer regulation by stathmin I & $9.96 \times 10^{-1}$ & CALML5 and PPPIRI4B \\
\hline Calcium signaling & 1.05 & CALML5 and TPM3 \\
\hline Calcium-induced T-lymphocyte apoptosis & $7.81 \times 10^{-1}$ & CALML5 \\
\hline cAMP-mediated signaling & $3.35 \times 10^{-1}$ & CALML5 \\
\hline Cardiac hypertrophy signaling & $8.85 \times 10^{-1}$ & PLCDI and CALML5 \\
\hline Cardiac $\beta$-adrenergic signaling & $5.03 \times 10^{-1}$ & PPPIRI4B \\
\hline Caveolar-mediated endocytosis signaling & $7.35 \times 10^{-1}$ & COPA \\
\hline CCR3 signaling in eosinophils & $5.5 \times 10^{-1}$ & CALML5 \\
\hline CCR5 signaling in macrophages & $7.52 \times 10^{-1}$ & CALML5 \\
\hline CD28 signaling in T helper cells & $5.47 \times 10^{-1}$ & CALML5 \\
\hline CDK5 signaling & $6.12 \times 10^{-1}$ & PPPIRI4B \\
\hline Cellular effects of sildenafil (Viagra) & 1.29 & PLCDI and CALML5 \\
\hline Chemokine signaling & $7.4 \times 10^{-1}$ & CALML5 \\
\hline Citrulline biosynthesis & 1.65 & GLS \\
\hline Clathrin-mediated endocytosis signaling & $3.89 \times 10^{-1}$ & CLTA \\
\hline Corticotropin releasing hormone signaling & $5.66 \times 10^{-1}$ & CALML5 \\
\hline CREB signaling in neurons & 1.08 & PLCDI and CALML5 \\
\hline CTLA4 signaling in cytotoxic T-lymphocytes & $6.57 \times 10^{-1}$ & CLTA \\
\hline Dendritic cell maturation & $4 \times 10^{-1}$ & PLCDI \\
\hline D-myo-inositol $(3,4,5,6)$-tetrakisphosphate biosynthesis & $5.23 \times 10^{-1}$ & PPPIRI4B \\
\hline D-myo-inositol-5-phosphate metabolism & 1.22 & PLCDI and PPPIRI4B \\
\hline D-myo-inositol $(I, 4,5)$-trisphosphate biosynthesis & 1.13 & PLCDI \\
\hline D-myo-inositol $(I, 4,5,6)$-tetrakisphosphate biosynthesis & $5.23 \times 10^{-1}$ & PPPIRI4B \\
\hline Dopamine receptor signaling & $7.04 \times 10^{-1}$ & PPPIRI4B \\
\hline Dopamine-DARPP32 feedback in cAMP signaling & 1.98 & PLCDI, CALML5, and PPPIRI4B \\
\hline EIF2 signaling & 7.34 & $\begin{array}{l}\text { RPS28, RPL22, EIF4A3, RPL29, RPL2I, } \\
\text { RPSI2, RPSI4, and RPLIOA }\end{array}$ \\
\hline Endothelin-I signaling & $4.14 \times 10^{-1}$ & PLCDI \\
\hline eNOS signaling & 1.22 & CALML5 and HSP90AAI \\
\hline Erk/MAPK signaling & $3.86 \times 10^{-1}$ & PPPIRI4B \\
\hline Eumelanin biosynthesis & 1.95 & MIF \\
\hline Fatty acid biosynthesis initiation II & 2.25 & FASN \\
\hline fMLP signaling in neutrophils & $5.8 \times 10^{-1}$ & CALML5 \\
\hline $\mathrm{FXR/RXR}$ activation & $5.2 \times 10^{-1}$ & FASN \\
\hline Gap junction signaling & $4.49 \times 10^{-1}$ & PLCDI \\
\hline
\end{tabular}


Table 6 (Continued)

\begin{tabular}{|c|c|c|}
\hline Ingenuity canonical pathways & $\log P$ & Protein molecules \\
\hline Glioblastoma multiforme signaling & $4.7 \times 10^{-1}$ & PLCDI \\
\hline Glioma signaling & $6.28 \times 10^{-1}$ & CALML5 \\
\hline Glucocorticoid receptor signaling & $2.81 \times 10^{-1}$ & HSP90AAI \\
\hline Gluconeogenesis I & 1.17 & ALDOA \\
\hline Glutamate degradation II & 2.07 & GOT2 \\
\hline Glutamate receptor signaling & 1.95 & CALML5 and GLS \\
\hline Glutamine degradation I & 2.25 & GLS \\
\hline Glutathione biosynthesis & 2.07 & GCLC \\
\hline Glycolysis I & 1.17 & ALDOA \\
\hline Granulocyte adhesion and diapedesis & $4.04 \times 10^{-1}$ & MSN \\
\hline Granzyme A signaling & 4.63 & HISTIHIB, HISTIHIC, and HISTIHIE \\
\hline Guanine and guanosine salvage I & 2.25 & PNP \\
\hline Guanosine nucleotides degradation III & 1.44 & PNP \\
\hline $\mathrm{G}_{\alpha 0}$ signaling & $4.68 \times 10^{-1}$ & CALML5 \\
\hline HIFI $\alpha$ signaling & $5.97 \times 10^{-1}$ & HSP90AAI \\
\hline Huntington's disease signaling & $8.63 \times 10^{-1}$ & CLTA and GLS \\
\hline Hypoxia signaling in the cardiovascular system & $7.75 \times 10^{-1}$ & HSP90AAI \\
\hline ICOS-ICOSL signaling in T helper cells & $5.8 \times 10^{-1}$ & CALML5 \\
\hline IL-8 signaling & $3.93 \times 10^{-1}$ & CSTB \\
\hline ILK signaling & 1.81 & KRTI8, TMSBI0/TMSB4X, and PPPIRI4B \\
\hline ¡NOS signaling & $9.32 \times 10^{-1}$ & CALML5 \\
\hline Insulin receptor signaling & $5.01 \times 10^{-1}$ & PPPIRI4B \\
\hline L-cysteine degradation I & 1.95 & GOT2 \\
\hline Leptin signaling in obesity & $7.24 \times 10^{-1}$ & PLCDI \\
\hline Leukocyte extravasation signaling & $3.67 \times 10^{-1}$ & MSN \\
\hline LXR/RXR activation & $5.38 \times 10^{-1}$ & FASN \\
\hline Melatonin signaling & 1.78 & PLCDI and CALML5 \\
\hline MIF regulation of innate immunity & $9.61 \times 10^{-1}$ & MIF \\
\hline MIF-mediated glucocorticoid regulation & 1.05 & MIF \\
\hline Mitochondrial dysfunction & $4.16 \times 10^{-1}$ & VDAC2 \\
\hline Mitotic roles of polo-like kinase & $7.69 \times 10^{-1}$ & HSP90AAI \\
\hline mTOR signaling & 3.73 & RPS28, EIF4A3, FKBPIA, RPSI2, and RPSI4 \\
\hline Neuregulin signaling & $6.57 \times 10^{-1}$ & HSP90AAI \\
\hline Neuropathic pain signaling in dorsal horn neurons & $6.08 \times 10^{-1}$ & PLCDI \\
\hline Nitric oxide signaling in the cardiovascular system & 1.5 & CALML5 and HSP90AAI \\
\hline nNOS signaling in skeletal muscle cells & 1.38 & CALML5 \\
\hline nNOS signaling in neurons & $9.05 \times 10^{-1}$ & CALML5 \\
\hline Nrf2-mediated oxidative stress response & 1.85 & SODI, DNAJBII, and GCLC \\
\hline Nur77 signaling in T-lymphocytes & $8.28 \times 10^{-1}$ & CALML5 \\
\hline Oncostatin M signaling & 1.04 & MT2A \\
\hline$P_{2} Y$ purigenic receptor signaling pathway & $5.44 \times 10^{-1}$ & PLCDI \\
\hline p70S6K signaling & $5.44 \times 10^{-1}$ & PLCDI \\
\hline Palmitate biosynthesis I (animals) & 2.25 & FASN \\
\hline Pentose phosphate pathway & 1.51 & G6PD \\
\hline Pentose phosphate pathway (oxidative branch) & 1.85 & G6PD \\
\hline Phenylalanine degradation IV (mammalian, via side chain) & 1.41 & GOT2 \\
\hline Phospholipase C signaling & $3.07 \times 10^{-1}$ & CALML5 \\
\hline Phospholipases & $8.28 \times 10^{-1}$ & PLCDI \\
\hline PI3K signaling in B-lymphocytes & 1.3 & PLCDI and CALML5 \\
\hline PI3K/Akt signaling & $5.32 \times 10^{-1}$ & HSP90AAI \\
\hline PPAR signaling & $6.32 \times 10^{-1}$ & HSP90AAI \\
\hline PPARA/RXRA activation & 3.83 & $\begin{array}{l}\text { PLCDI, HELZ2, FASN, HSP90AAI, } \\
\text { and GOT2 }\end{array}$ \\
\hline $\begin{array}{l}\text { Production of nitric oxide and reactive oxygen species } \\
\text { in macrophages }\end{array}$ & $3.98 \times 10^{-1}$ & PPPIRI4B \\
\hline Prostate cancer signaling & $6.84 \times 10^{-1}$ & HSP90AAI \\
\hline
\end{tabular}


Table 6 (Continued)

\begin{tabular}{|c|c|c|}
\hline Ingenuity canonical pathways & $\log P$ & Protein molecules \\
\hline Protein kinase $A$ signaling & 3.15 & $\begin{array}{l}\text { PLCDI, HISTIHIB, HISTIHIC, CALML5, } \\
\text { HISTIHIE, and PPPIRI4B }\end{array}$ \\
\hline Protein ubiquitination pathway & $7.92 \times 10^{-1}$ & DNAJBII and HSP90AAI \\
\hline Purine nucleotides degradation II (aerobic) & 1.26 & PNP \\
\hline $\begin{array}{l}\text { Purine ribonucleosides degradation to ribose-I- } \\
\text { phosphate }\end{array}$ & 1.65 & PNP \\
\hline RANK signaling in osteoclasts & $6.57 \times 10^{-1}$ & CALML5 \\
\hline Regulation of actin-based motility by Rho & $6.44 \times 10^{-1}$ & PFNI \\
\hline Regulation of EIF4 and p70S6K signaling & 3.12 & RPS28, EIF4A3, RPSI2, and RPSI4 \\
\hline Regulation of IL-2 expression in activated and anergic & $6.99 \times 10^{-1}$ & CALML5 \\
\hline \multicolumn{3}{|l|}{ T-lymphocytes } \\
\hline RhoA signaling & 1.33 & PFNI and MSN \\
\hline RhoGDI signaling & $4.12 \times 10^{-1}$ & MSN \\
\hline Role of macrophages, fibroblasts, and endothelial cells & 1.29 & PLCDI, CALML5, and MIF \\
\hline \multicolumn{3}{|l|}{ in rheumatoid arthritis } \\
\hline Role of NFAT in cardiac hypertrophy & 1.04 & PLCDI and CALML5 \\
\hline Role of NFAT in regulation of the immune response & $4.16 \times 10^{-1}$ & CALML5 \\
\hline $\begin{array}{l}\text { Role of osteoblasts, osteoclasts and chondrocytes in } \\
\text { rheumatoid arthritis }\end{array}$ & $3.35 \times 10^{-1}$ & CALML5 \\
\hline Signaling by Rho Family GTPases & $3.14 \times 10^{-1}$ & MSN \\
\hline Sperm motility & 1.35 & PLCDI and CALML5 \\
\hline Sphingosine-I-phosphate signaling & $5.76 \times 10^{-1}$ & PLCDI \\
\hline Stearate biosynthesis I (animals) & 1.03 & FASN \\
\hline Sucrose degradation V (mammalian) & 1.6 & ALDOA \\
\hline Superoxide radicals degradation & 1.78 & SODI \\
\hline Superpathway of citrulline metabolism & 1.38 & GLS \\
\hline Superpathway of inositol phosphate compounds & $9.96 \times 10^{-1}$ & PLCDI and PPPIRI4B \\
\hline Superpathway of methionine degradation & 1.08 & GOT2 \\
\hline Synaptic long-term depression & $4.8 \times 10^{-1}$ & PLCDI \\
\hline Synaptic long-term potentiation & 2.34 & PLCDI, CALML5, and PPPIRI4B \\
\hline Systemic lupus erythematosus signaling & $3.32 \times 10^{-1}$ & LSM2 \\
\hline T-cell receptor signaling & $6.2 \times 10^{-1}$ & CALML5 \\
\hline Telomerase signaling & $6.12 \times 10^{-1}$ & HSP90AAI \\
\hline Thrombin signaling & $3.79 \times 10^{-1}$ & PLCDI \\
\hline$T R / R X R$ activation & $6.7 \times 10^{-1}$ & FASN \\
\hline tRNA charging & $9.82 \times 10^{-1}$ & GARS \\
\hline Urate biosynthesis/inosine $5^{\prime}$-phosphate degradation & 1.41 & PNP \\
\hline UVA-induced MAPK signaling & $6.57 \times 10^{-1}$ & PLCDI \\
\hline Virus entry via endocytic pathways & $6.53 \times 10^{-1}$ & CLTA \\
\hline Xanthine and xanthosine salvage & 2.55 & PNP \\
\hline Xenobiotic metabolism signaling & $7.51 \times 10^{-1}$ & HSP90AAI and GCLC \\
\hline$\alpha$-adrenergic signaling & $6.61 \times 10^{-1}$ & CALML5 \\
\hline
\end{tabular}

Abbreviations: Akt, protein kinase B; ALDOA, fructose-bisphosphate aldolase A; AMPK, AMP-activated protein kinase; cAMP, cyclic adenosine monophosphate; CALML, calmodulin-like protein; CCR, C-C chemokine receptor; CDK, cyclin-dependent kinase; CREB, cAMP response element-binding protein; CTLA4, cytotoxic T-lymphocyte antigen 4; G6PD, glucose-6-phosphate I-dehydrogenase; EIF, eukaryotic initiation factor; FASN, fatty acid synthase; fMLP, N-formyl-methionyl-leucyl-phenylalanine; FXR, farnesoid X receptor; HIF, hypoxia-inducible factor; HSP, heat shock protein; ICOS, inducible co-stimulator; ICOSL, ICOS ligand; IL, interleukin; ILK, integrin-linked kinase; iNOS, inducible nitric oxide synthase; LXR, liver X receptor; MAPK, mitogen-activated protein kinase; MIF, migration inhibitory factor; mTOR, mammalian target of rapamycin; NFAT, nuclear factor of activated T-cell; nNOS, neuronal nitric oxide synthase; Nrf2, Kelch-like ECH-associated protein I and Cullin 3; PI3K, phosphoinositide 3-kinase; PLB, plumbagin; PLCD, I-phosphatidylinositol 4,5-bisphosphate phosphodiesterase- $\delta$ I; PPAR, peroxisome proliferator-activated receptor; RANK, receptor activator of nuclear factor-KB; RhoA, Ras homolog gene family, member A; RhoGDI, Rho GDP-dissociation inhibitor; RPS, ribosomal protein S; RXR, retinoid X receptor; SOD, superoxide dismutase; TR, thyroid hormone receptor; UVA, ultraviolet $A$.

a central role in the antiproliferative and autophagy-inducing effects of PLB in PC-3 and DU145 cells.

Taken together, our proteomic study has revealed that a number of important proteins and their associated signaling pathways are regulated in PC-3 and DU145 cells in response to PLB. These cellular signaling pathways play pivotal roles in the regulation of cell cycle, apoptosis, autophagy, EMT, and oxidative stress with the involvement of a number of critical functional proteins, such as PI3K, mTOR, Akt, MAPK, CDKs, cytochrome c, and E-cadherin. 


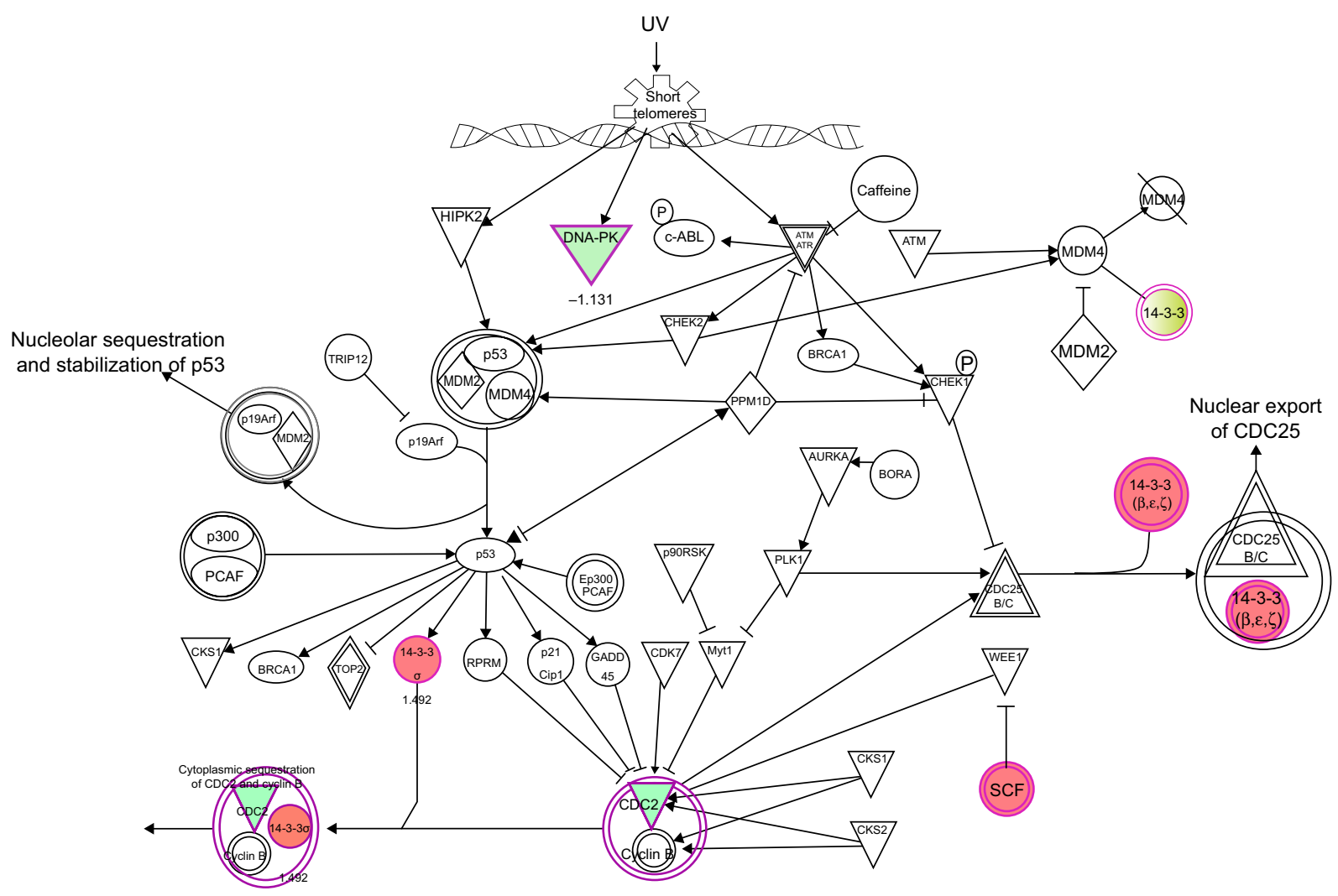

Figure 10 PLB regulates cell cycle at $G_{2} / M$ checkpoint in PC-3 cells.

Notes: PC-3 cells were treated with $5 \mu$ M PLB for 24 hours and the protein samples were subject to quantitative proteomic analysis. Red indicates an upregulation; green indicates a downregulation; brown indicates a predicted activation. The intensity of green and red molecule colors indicates the degree of down- or upregulation, respectively. Solid arrows indicates direct interaction.

Abbreviations: PLB, plumbagin; UV, ultraviolet.

\section{Verification of molecular targets of PLB in PC-3 and DUI 45 cells by Western blot assay}

Our above bioinformatic and quantitative proteomic studies have predicted and shown that PLB can modulate a number of signaling pathways related to cell proliferation, cell migration, cell death, and cell survival. In the next set of functional validation experiments, in order to further verify the quantitative proteomic data, we tested how PLB affected the cell cycle, apoptosis, autophagy, EMT, and redox homeostasis and the related signaling pathways in PC-3 and DU145 cells.

PLB inhibits the proliferation of PC-3 and DUI45 cells, and induces G2/M arrest in PC-3 cells and GI arrest in DUI 45 cells via regulation of cyclin $\mathrm{BI}$, cyclin DI, CDKI/CDC2, CDK2, p2 I Wafl/CipI, p27 Kipl, and p53

First, we examined the effect of PLB on cell cycle distribution using a flow cytometer in both cell lines. PLB showed differential effects on the cell cycle distribution in PC-3 and DU145 cells (Figure 17A and B). In PC-3 cells, PLB significantly induced a $\mathrm{G}_{2} / \mathrm{M}$ phase arrest. Compared with the control cells (20.1\%), the percentage of PC-3 cells in $\mathrm{G}_{2} / \mathrm{M}$ phase was increased in a concentration-dependent manner after PLB treatment (Figure 17A and B). The percentage was 25.4\%, $28.1 \%, 32.3 \%$, and $38.5 \%$ when treated with PLB at $0.1,1$, 5 , and $10 \mu \mathrm{M}$, respectively. PLB significantly decreased the percentage of PC-3 cells in $\mathrm{G}_{1}$ phase in comparison to the control cells. The basal level of PC-3 cells in $\mathrm{G}_{1}$ phase was $60.9 \%$; after treatment with PLB at $0.1,1,5$, and $10 \mu \mathrm{M}$ for 24 hours, the percentage of PC-3 cells in $\mathrm{G}_{1}$ phase was $53.2 \%, 53.9 \%, 52.5 \%$, and $47.5 \%$, respectively. However, there was no significant difference observed in the number of cells in S phase in PC-3 cells when treated with PLB (Figure 17A and B).

We further conducted separate experiments to evaluate the effect of PLB treatment at $5 \mu \mathrm{M}$ on cell cycle distribution in PC-3 cells over 72 hours. Compared to the control cells, the percentage of PC-3 cells in $\mathrm{G}_{2} / \mathrm{M}$ phase was increased from $23.5 \%$ at basal level to $28.6 \%, 28.8 \%$, and $28.9 \%$ after 4,8 , and 12 hours treatment with $5 \mu \mathrm{M}$ PLB and declined to $25.4 \%, 18.1 \%$, and $17.6 \%$ after 24,48 , and 72 hours 


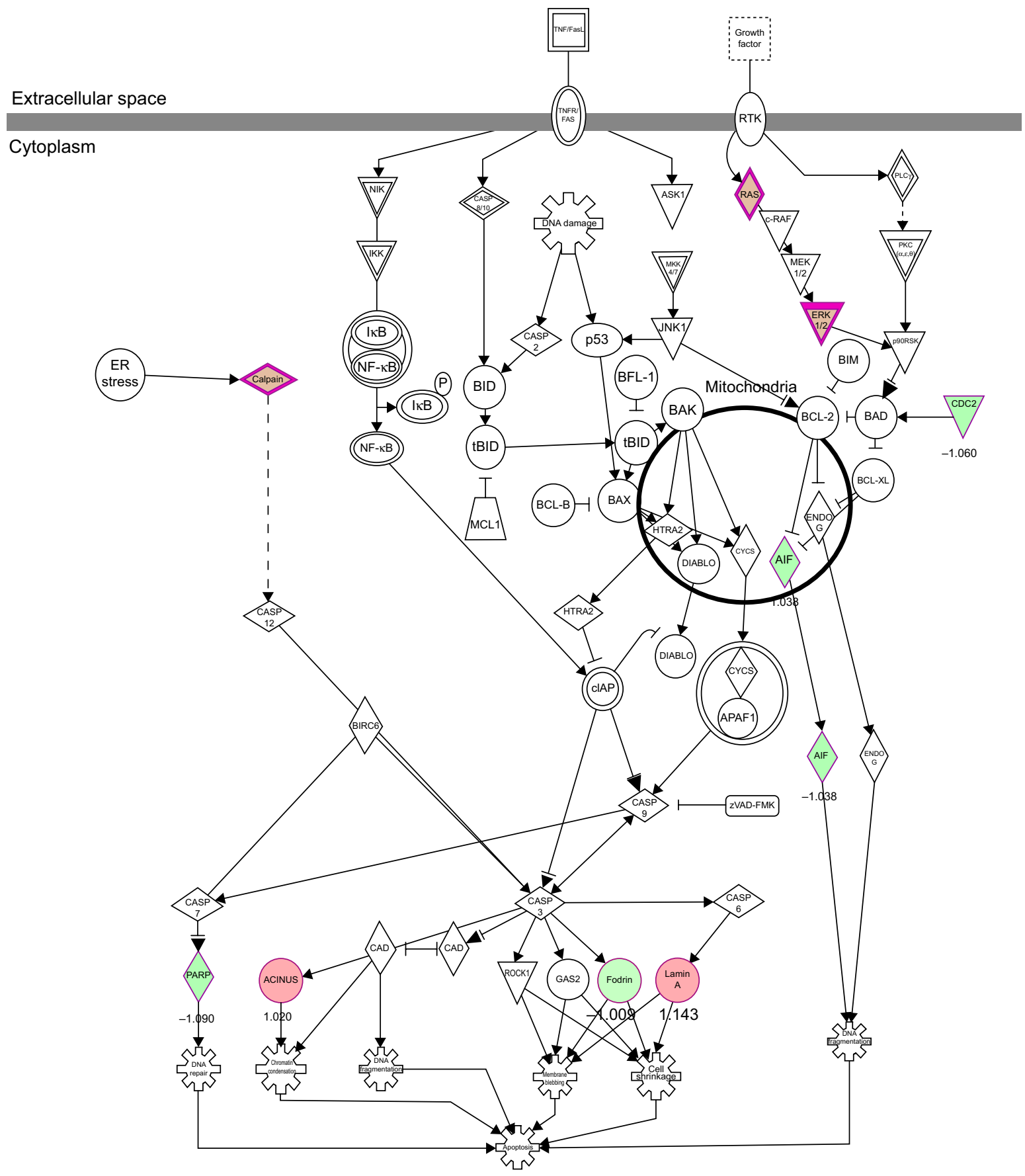

Figure II PLB regulates apoptosis signaling pathway in PC-3 cells.

Notes: PC-3 cells were treated with $5 \mu$ M PLB for 24 hours and the protein samples were subject to quantitative proteomic analysis. Red indicates an upregulation; green indicates a downregulation. The intensity of green and red molecule colors indicates the degree of down- or upregulation, respectively. Solid arrows indicate direct interaction and dashed arrows indicate indirect interaction.

Abbreviation: PLB, plumbagin.

treatment of PLB, respectively (Figure 18A and B). While $5 \mu \mathrm{M}$ PLB treatment decreased the percentage of PC-3 cells in $\mathrm{G}_{1}$ phase from $62.9 \%$ at basal level to $55.9 \%, 57.0 \%$, and $56.0 \%$ after 4,8 , and 12 hours treatment and was increased to $59.3 \%, 72.4 \%$, and $79.4 \%$ after 24,48 , and 72 hours drug treatment, respectively (Figure 18A and B). There was a significant decrease in the percentage of PC-3 cells in S phase after treatment with PLB for 48 and 72 hours.

PLB exhibited a differential effect on the cell cycle distribution of DU145 cells. PLB significantly induced $\mathrm{G}_{1}$ arrest with an increase in the percentage of DU145 cells in $\mathrm{G}_{1}$ phase (Figure 17A and B). In comparison to the control 


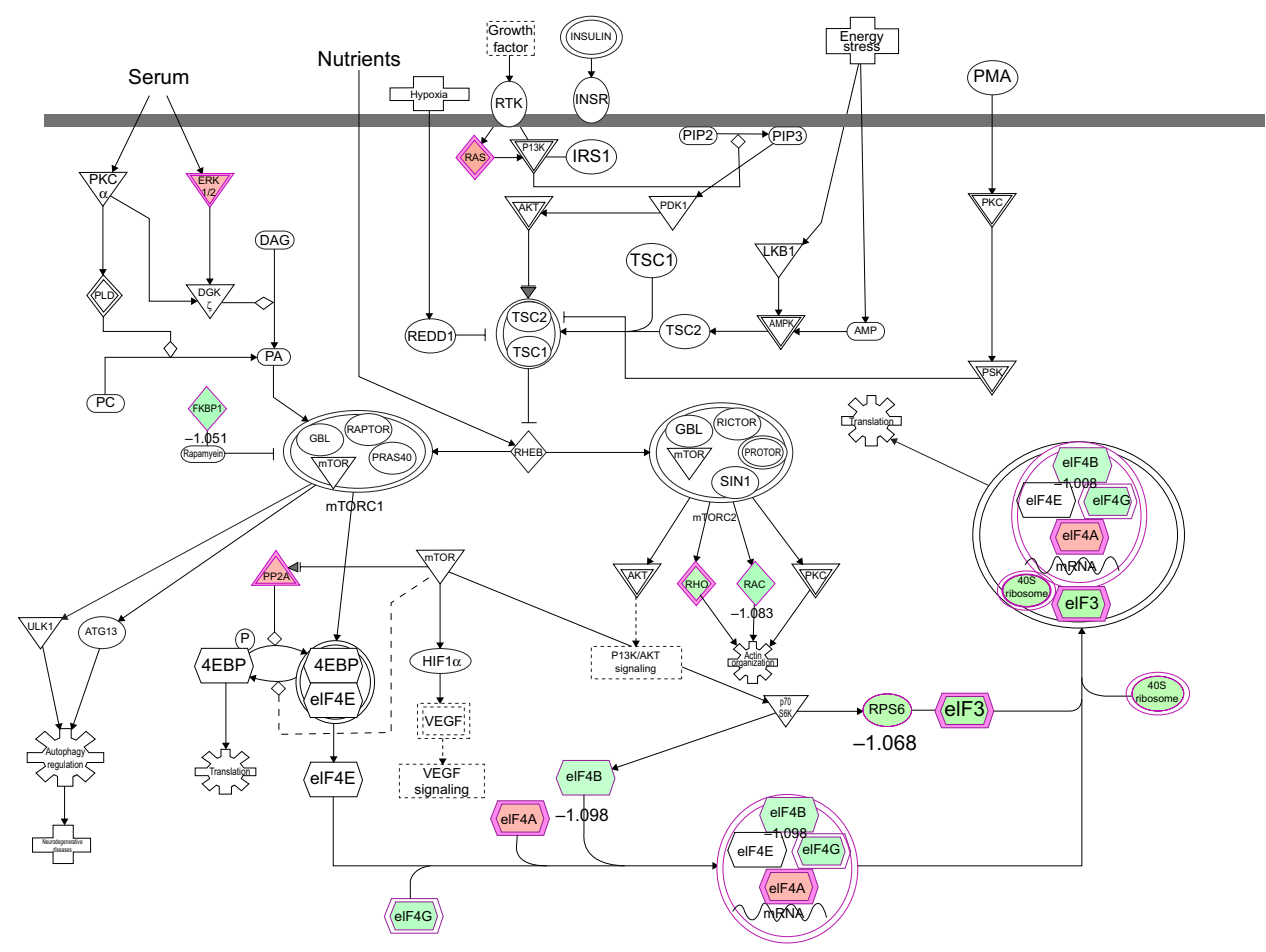

Figure 12 mTOR signaling pathway regulated by PLB in PC-3 cells.

Notes: PC-3 cells were treated with $5 \mu$ M PLB for 24 hours and the protein samples were subject to quantitative proteomic analysis. Red indicates an upregulation; green indicates a downregulation. The intensity of green and red molecule colors indicates the degree of down- or upregulation, respectively. Solid arrows indicate direct interaction and dashed arrows indicate indirect interaction.

Abbreviation: PLB, plumbagin.

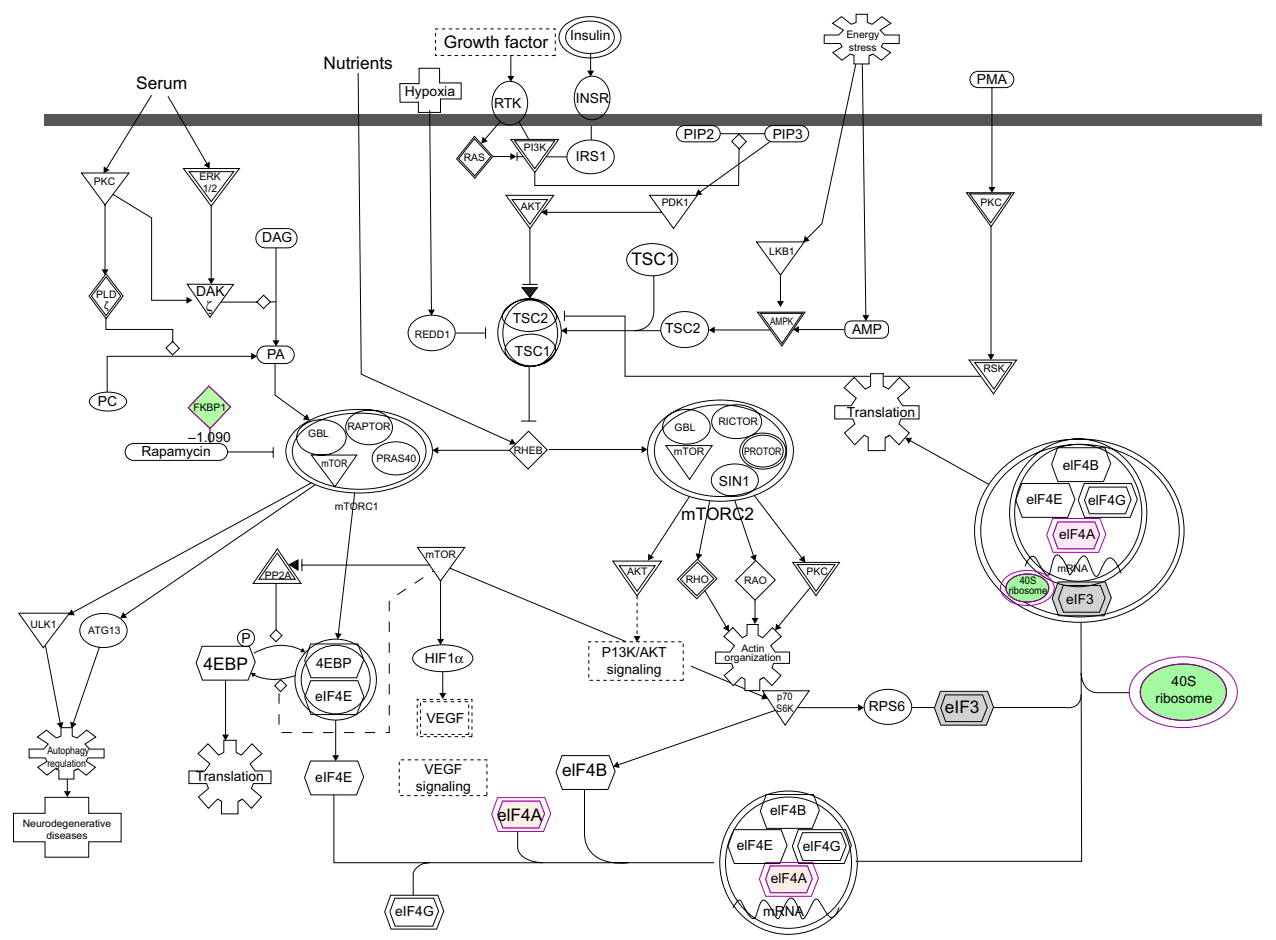

Figure 13 mTOR signaling pathway regulated by PLB in DUI45 cells.

Notes: DUI45 cells were treated with $5 \mu$ M PLB for 24 hours and the protein samples were subject to quantitative proteomic analysis. Red indicates an upregulation; green indicates a downregulation. The intensity of green and red molecule colors indicates the degree of down- or upregulation, respectively. Solid arrows indicate direct interaction and dashed arrows indicate indirect interaction.

Abbreviation: PLB, plumbagin. 


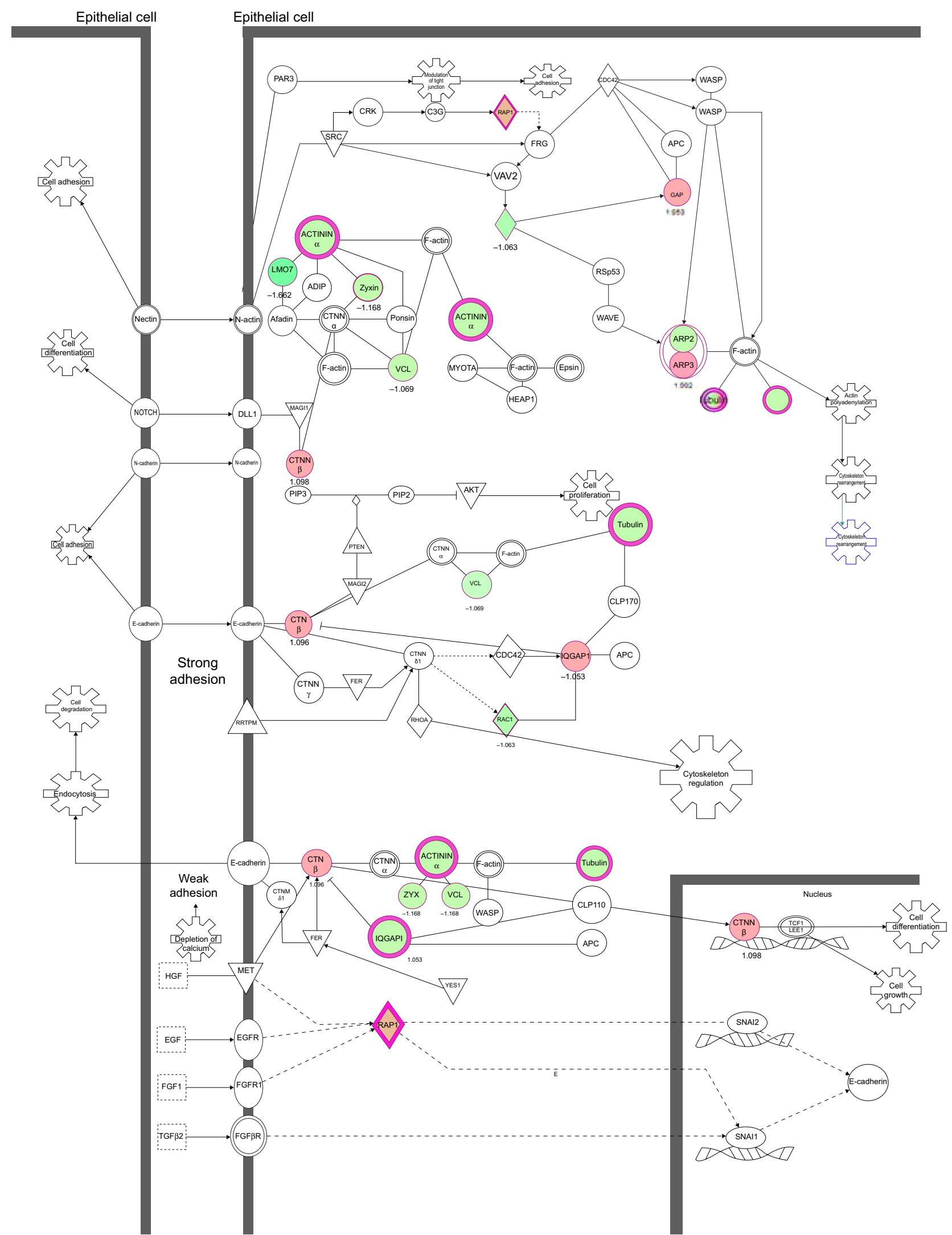

Figure 14 PLB regulates epithelial adherent junction signaling pathway in PC-3 cells.

Notes: PC-3 cells were treated with $5 \mu$ M PLB for 24 hours and the protein samples were subject to quantitative proteomic analysis. Red indicates an upregulation; green indicates a downregulation; brown indicates a predicted activation. The intensity of green and red molecule colors indicates the degree of down- or upregulation, respectively. Solid arrows indicate direct interaction and dashed arrows indicate indirect interaction.

Abbreviation: PLB, plumbagin. 


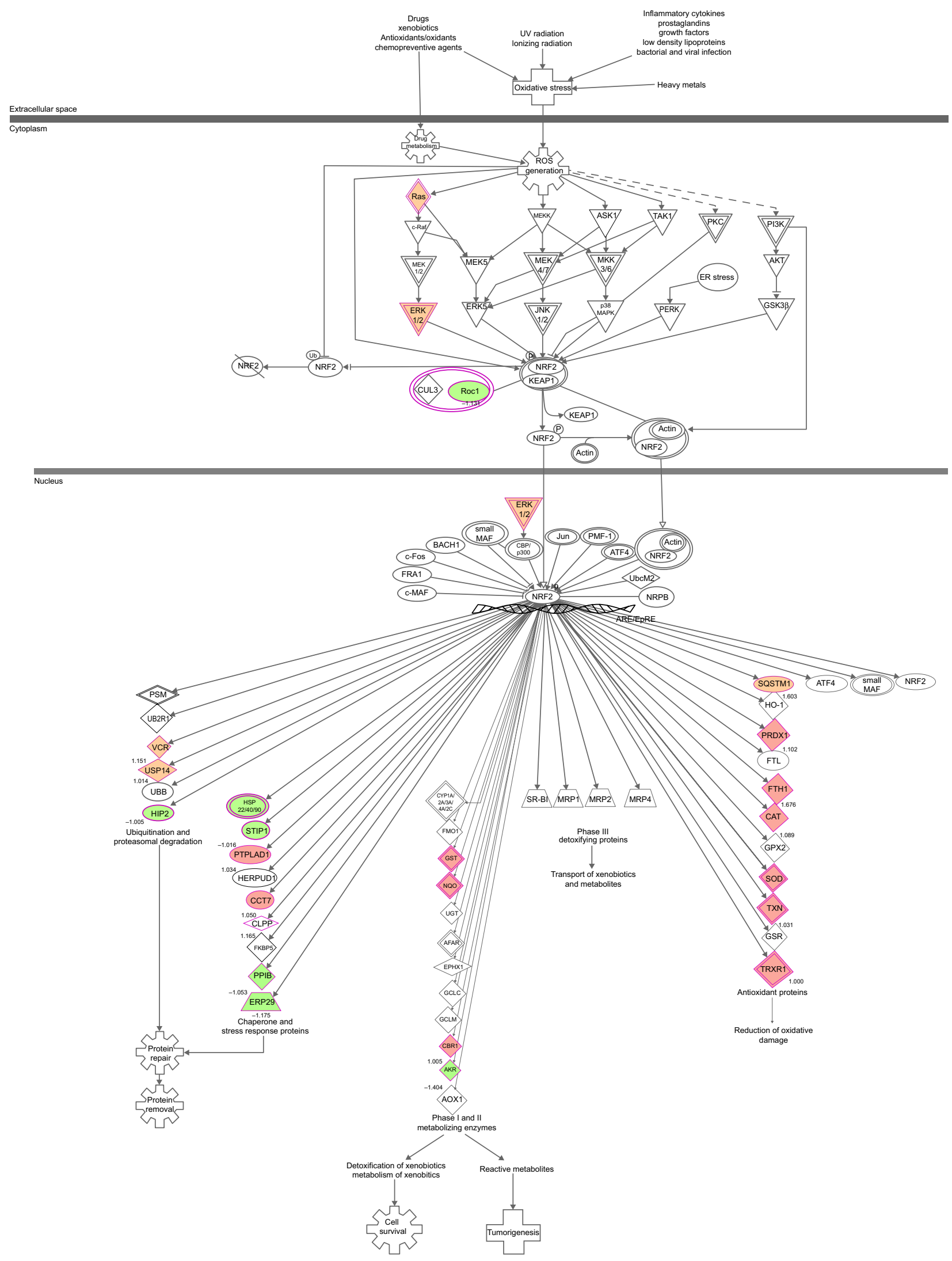

Figure I5 PLB-regulated Nrf2-mediated oxidative stress response in PC-3 cells.

Notes: PC-3 cells were treated with $5 \mu$ M PLB for 24 hours and the protein samples were subject to quantitative proteomic analysis. Red indicates an upregulation; green indicates a downregulation; brown indicates a predicted activation. The intensity of green and red molecule colors indicates the degree of down- or upregulation, respectively. Solid arrows indicate direct interaction and dashed arrows indicate indirect interaction.

Abbreviations: PLB, plumbagin; UV, ultraviolet. 


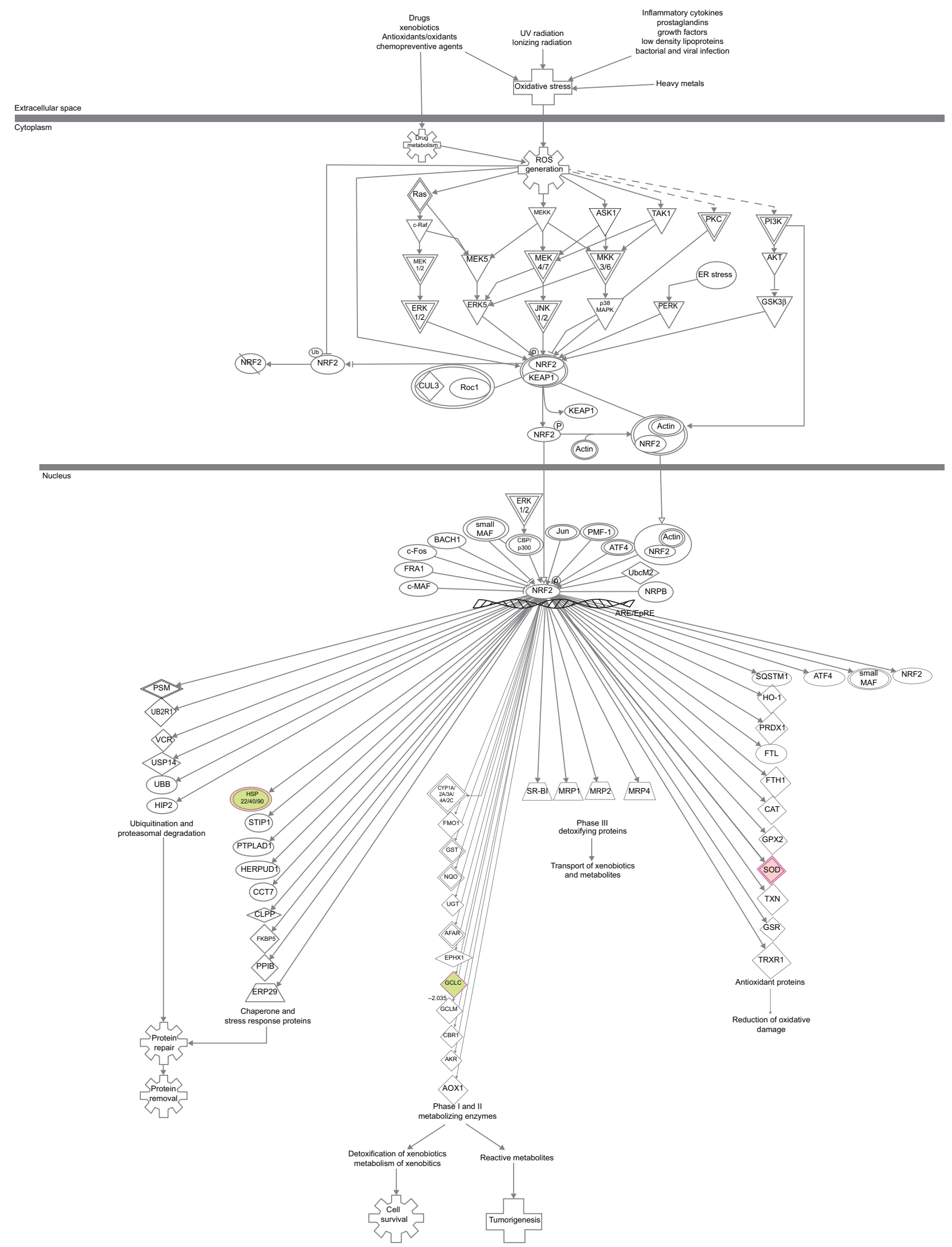

Figure 16 PLB-regulated Nrf2-mediated oxidative stress response in DUI 45 cells.

Notes: DUI45 cells were treated with $5 \mu$ M PLB for 24 hours and the protein samples were subject to quantitative proteomic analysis. Red indicates an upregulation; green indicates a downregulation. The intensity of green and red molecule colors indicates the degree of down- or upregulation, respectively. Solid arrows indicate direct interaction and dashed arrows indicate indirect interaction.

Abbreviations: PLB, plumbagin; UV, ultraviolet. 
Table 7 Top five canonical pathways regulated by PLB in PC-3 cells

\begin{tabular}{lll}
\hline $\begin{array}{l}\text { Ingenuity canonical } \\
\text { pathways }\end{array}$ & P-value & Ratio (H/L) \\
\hline EIF2 signaling & $9.19 \times 10^{-66}$ & $94 / 201(0.468)$ \\
$\begin{array}{l}\text { Regulation of EIF4 } \\
\text { and p70S6K signaling }\end{array}$ & $6.24 \times 10^{-34}$ & $59 / 175(0.337)$ \\
mTOR signaling & $9.78 \times 10^{-23}$ & $54 / 213(0.254)$ \\
$\begin{array}{l}\text { Protein ubiquitination } \\
\text { pathway }\end{array}$ & $4.1 \times 10^{-22}$ & $62 / 270(0.23)$ \\
Mitochondrial dysfunction & $5.53 \times 10^{-20}$ & $47 / 215(0.219)$ \\
\hline
\end{tabular}

Abbreviations: EIF, eukaryotic initiation factor; $\mathrm{H}$, medium supplemented with stable isotope-labeled L-arginine and L-lysine; L, medium supplemented with normal L-arginine and L-lysine; mTOR, mammalian target of rapamycin; PLB, plumbagin.

cells $(57.3 \%)$, the percentage of DU145 cells in $\mathrm{G}_{1}$ phase was increased in a concentration-dependent manner. The values were $58.6 \%, 55.2 \%, 67.8 \%$, and $80.4 \%$ with the PLB treatment at concentrations of $0.1,1,5$, and $10 \mu \mathrm{M}$, respectively. A significant reduction of the number of cells in $\mathrm{G}_{2} / \mathrm{M}$ phase was also observed after PLB treatment for 24 hours. The percentage was decreased from $28.9 \%$ (control) to $13.8 \%$ (10 $\mu \mathrm{M}$ PLB). In addition, when DU145 cells were treated with PLB at 1 and $5 \mu \mathrm{M}$ for 24 hours, we observed a significant increase in the number of the cell population in $\mathrm{S}$ phase; however, incubation with $10 \mu \mathrm{M}$ of PLB reduced the cell population in S phase $(14.0 \%$ versus $5.9 \%)(P<0.001$; Figure $17 \mathrm{~A}$ and $\mathrm{B}$ ).

In addition, treatment of DU145 cells with $5 \mu \mathrm{M}$ PLB for $4,8,12,24,48$, or 72 hours significantly increased the percentage of cells in S phase from 7.3\% at basal level to $10.6 \%, 11.4 \%, 9.7 \%, 10.0 \%, 10.2 \%$, and $12.7 \%$, respectively (Figure 18A and B). Although there was no significant change in the percentage of DU145 cells in $\mathrm{G}_{2} / \mathrm{M}$ and $\mathrm{G}_{1}$ phase, there was an $8.8 \%$ and $22.9 \%$ decrease in the percentage of DU145 cells in $\mathrm{G}_{2} / \mathrm{M}$ phase observed when the cells were treated with $5 \mu \mathrm{M}$ PLB for 48 and 72 hours, respectively.

Table 8 Top five canonical pathways regulated by PLB in DUI45 cells

\begin{tabular}{lll}
\hline Ingenuity canonical pathways & P-value & Ratio (H/L) \\
\hline EIF2 signaling & $4.61 \times 10^{-8}$ & $8 / 185(0.043)$ \\
Granzyme A signaling & $2.33 \times 10^{-5}$ & $3 / 20(0.15)$ \\
PPAR- $\alpha / R X R \alpha$ activation & $1.47 \times 10^{-4}$ & $5 / 179(0.028)$ \\
mTOR signaling & $1.84 \times 10^{-4}$ & $5 / 188(0.027)$ \\
Protein kinase A signaling & $7.13 \times 10^{-4}$ & $6 / 384(0.016)$ \\
\hline
\end{tabular}

Abbreviations: EIF, eukaryotic initiation factor; $\mathrm{H}$, medium supplemented with stable isotope-labeled L-arginine and L-lysine; L, medium supplemented with normal L-arginine and L-lysine; mTOR, mammalian target of rapamycin; PLB, plumbagin; PPAR, peroxisome proliferator-activated receptor; $R X R$, retinoid $X$ receptor.
To explore the mechanisms for PLB-induced effects on cell cycle arrest in PC-3 and DU145 cells, the expression levels of key regulators responsible for $G_{1}$ and $G_{2}$ checkpoints were examined using Western blot assay. Cyclin B1 and $\mathrm{CDK} 1 / \mathrm{CDC} 2$ are two key regulators for $\mathrm{G}_{2}$ to $\mathrm{M}$ phase transition ${ }^{55}$ and thus their expression levels were determined in PC-3 cells. The expression of cyclin B1 was significantly suppressed in PC-3 cells with the treatment of PLB at concentrations of $0.1,1$, and $5 \mu \mathrm{M}$ for 24 hours $(P<0.001$; Figure 19A and B). In comparison to the control cells, the expression level of cyclin B1 in PC-3 cells was decreased 2.1-fold when treated with $5 \mu \mathrm{M}$ PLB for 24 hours. There was a $21.3 \%$ and $23.5 \%$ reduction in the expression level of CDK1/CDC2 in PC-3 cells incubated with PLB at 1 and $5 \mu \mathrm{M}$ for 24 hours, respectively $(P<0.05$ and $P<0.01$, respectively; Figure 19A and $\mathrm{B})$. However, there was no significant change in the expression level of CDK2 and cyclin D1 when PC-3 cells were treated with PLB at $0.1,1$, and $5 \mu \mathrm{M}$ for 24 hours $(P>0.05$; Figure 19A and B).

In DU145 cells, the expression levels of key regulators for $\mathrm{G}_{1}$ to $\mathrm{S}$ transition including CDK2 and cyclin D1 were determined. A significant inhibitory effect of PLB on the expression of CDK2 and cyclin D1 was observed, which was in a concentration-dependent manner (Figure 20A and B). Treatment of DU145 cells with PLB at 1 and $5 \mu \mathrm{M}$ for 24 hours resulted in a $42.1 \%$ and $42.0 \%$ decrease in the expression of cyclin D1, respectively $(P<0.05)$. A similar inhibitory effect on the expression of CDK2 was also observed $(P<0.01$; Figure $20 \mathrm{~A}$ and B). A low concentration of PLB $(0.1 \mu \mathrm{M})$ only slightly decreased the expression of cyclin D1 and CDK2 in DU145 cells. Incubation of DU145 cells with PLB did not significantly alter the expression level of cyclin $\mathrm{B} 1$ and $\mathrm{CDC} 2(P>0.05$; Figure $20 \mathrm{~A}$ and $\mathrm{B})$.

These results have demonstrated that PLB could downregulate $\mathrm{CDK} 1 / \mathrm{CDC} 2, \mathrm{CDK} 2$, cyclin $\mathrm{B} 1$, and cyclin $\mathrm{D} 1$ in PC-3 and DU145 cells with differential effects. This would contribute to the cell cycle arrest in both cell lines when exposed to PLB.

To further elucidate the mechanisms for the effect of PLB on cell cycle, the expression levels of $\mathrm{p} 21 \mathrm{Waf1} / \mathrm{Cip} 1$, p27 Kip1, and p53 in PC-3 and DU145 cells treated with PLB were determined using Western blot assay. The tumor suppressor protein $\mathrm{p} 21 \mathrm{Waf1} / \mathrm{Cip} 1$ acts as an inhibitor of cell cycle progression, and it serves to inhibit kinase activity and block progression through $\mathrm{G}_{1} / \mathrm{S}$ in association with $\mathrm{CDK} 2$ complexes. ${ }^{59}$ During cell cycle stages when CDC2/cyclin B or CDK2/cyclin A are active, p53 is phosphorylated and upregulates p21 Waf1/Cip1 transcription via a p53-responsive 


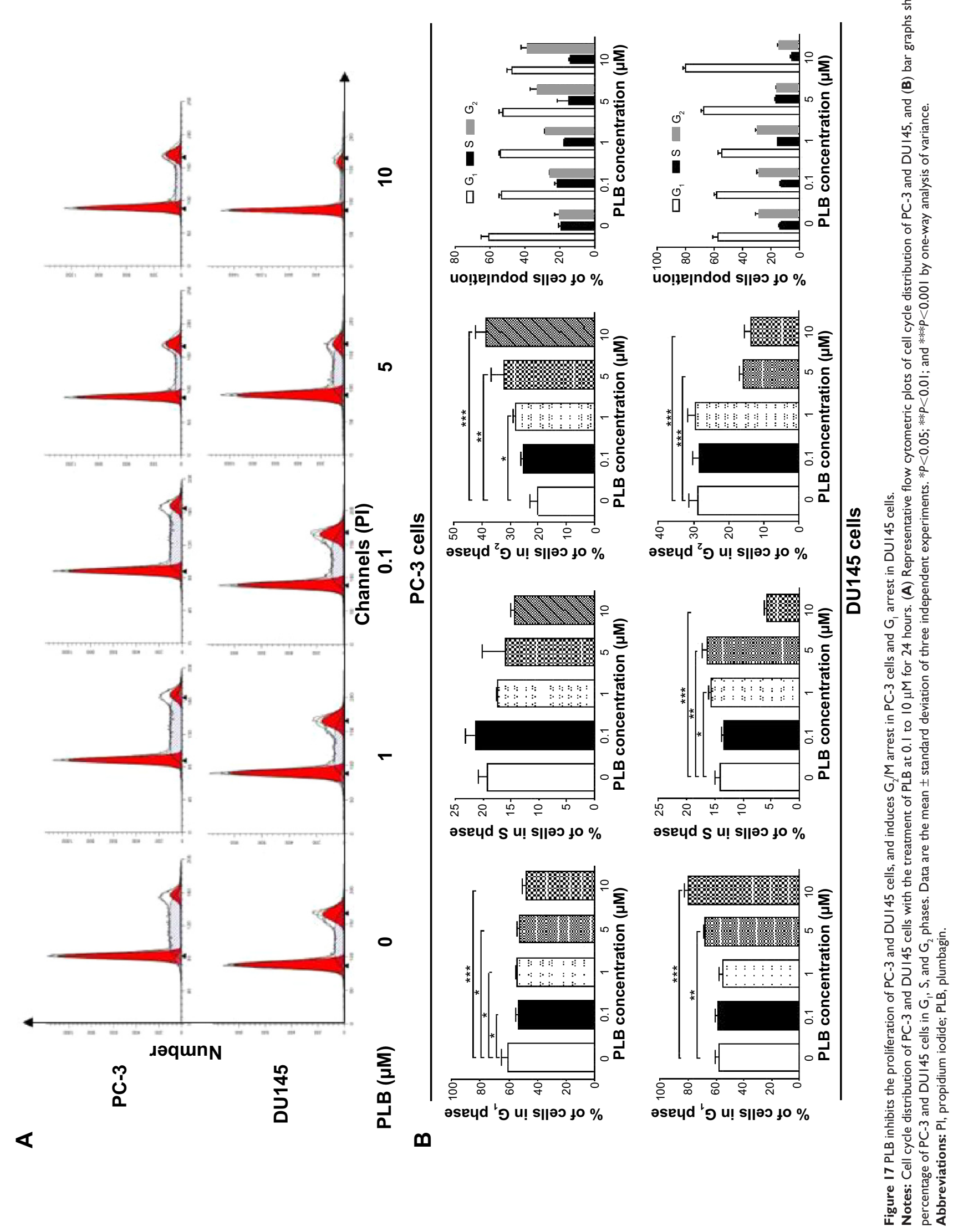




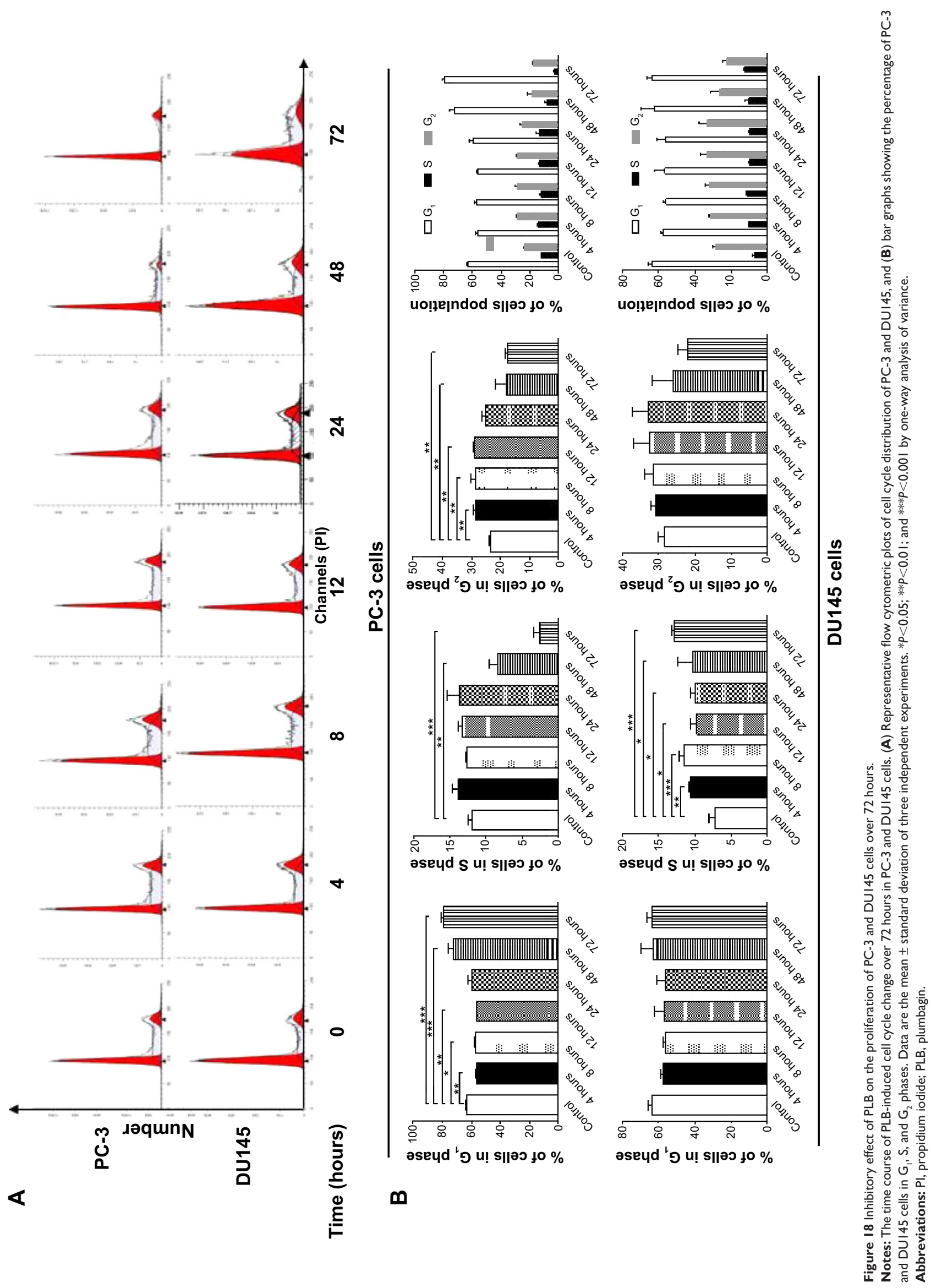


A
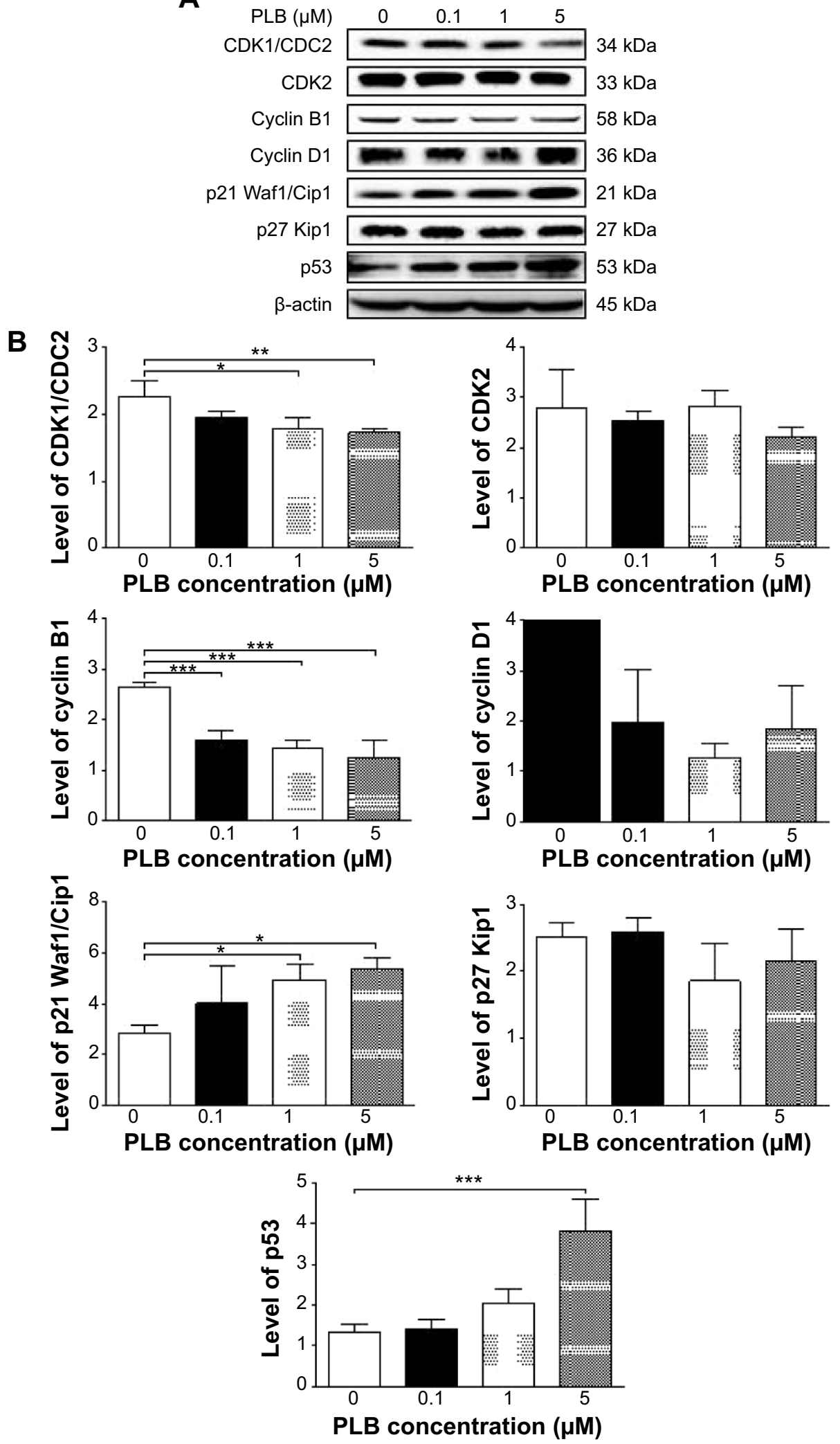

Figure 19 PLB regulates the expression of CDKI/CDC2, cyclin BI, CDK2, cyclin DI, p2I Wafl/CipI, p27 KipI, and p53 in PC-3 cells.

Notes: PC-3 cells were treated with PLB at 0.1 , I, and $5 \mu \mathrm{M}$ for 24 hours and protein samples were subject to Western blot assay. (A) Representative blots of CDKI/ CDC2, cyclin BI, CDK2, cyclin DI, p2I Wafl/Cipl, p27 KipI, p53, and $\beta$-actin in PC-3 cells, and (B) bar graphs showing the relative levels of CDKI/CDC2, cyclin BI, CDK2, cyclin DI, p2I Wafl/Cipl, p27 Kipl, and p53 in PC-3 cells. Data are the mean \pm standard deviation of three independent experiments. $* P<0.05$; $* * P<0.01$; and $* * * P<0.00 I$ by one-way analysis of variance. Abbreviation: PLB, plumbagin. 


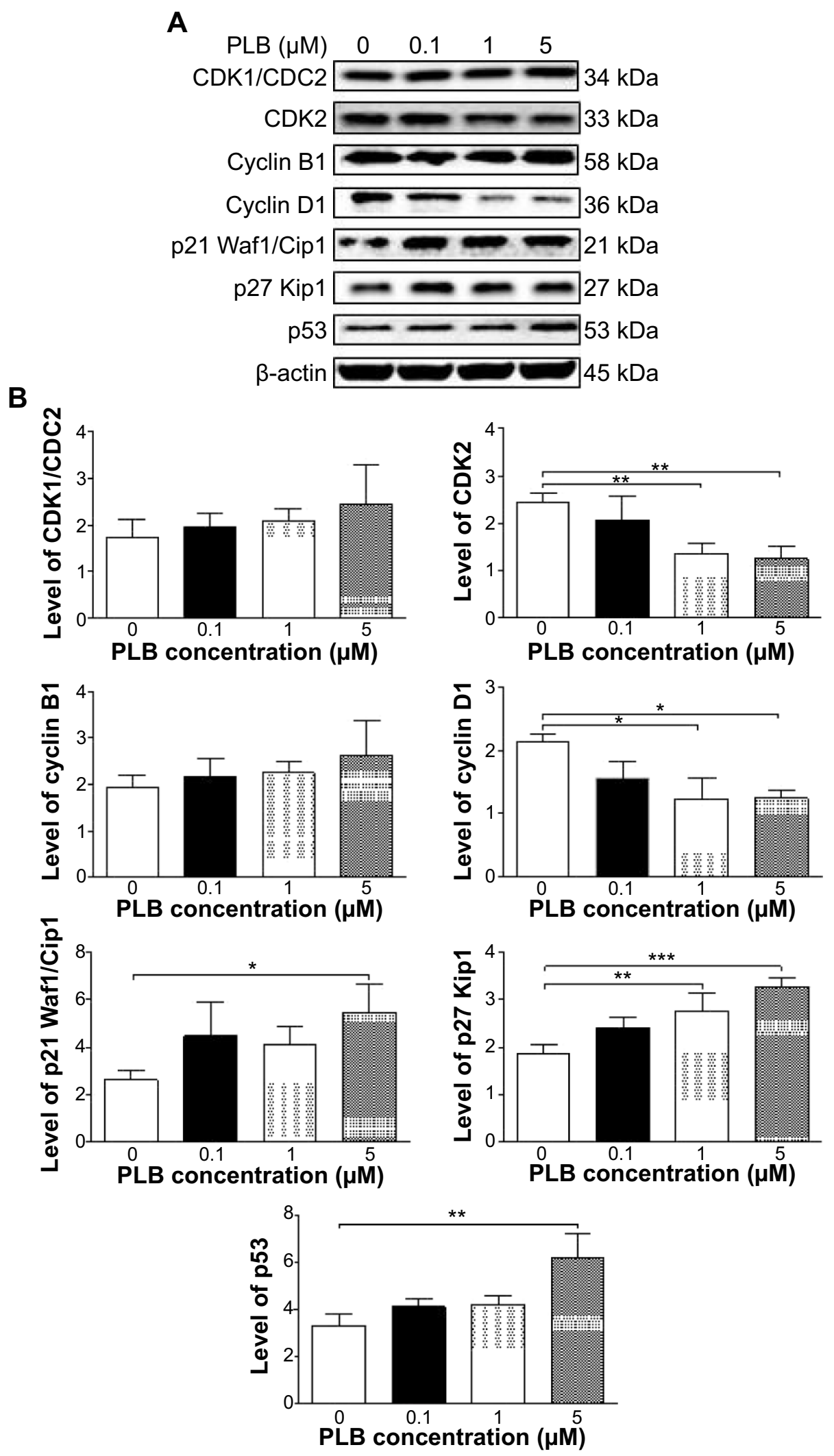

Figure 20 PLB regulates the expression of CDKI/CDC2, cyclin BI, CDK2, cyclin DI, p2I Wafl/CipI, p27 KipI, and p53 in DUI45 cells.

Notes: DUI 45 cells were treated with PLB at 0.1 , I, and $5 \mu \mathrm{M}$ for 24 hours and protein samples were subject to Western blot assay. (A) Representative blots of CDKI/ CDC2, cyclin BI, CDK2, cyclin DI, p2I Wafl/Cipl, p27 Kipl, p53, and $\beta$-actin in DUI45 cells, and (B) bar graphs showing the relative levels of CDKI/CDC2, cyclin BI, CDK2, cyclin DI, p2I Wafl/Cipl, p27 KipI, and p53 in DUI 45 cells. Data are the mean \pm standard deviation of three independent experiments. ${ }^{* P}<0.05 ; * * P<0.01$; and $* * * P<0.00$ I by one-way analysis of variance.

Abbreviation: PLB, plumbagin. 
element. p27 Kip1 is a member of the Cip/Kip family of cyclin-dependent kinase inhibitors. ${ }^{60}$ Like p57 Kip2 and p21 Waf1/Cip1, p27 Kip1 enforces the $\mathrm{G}_{1}$ restriction point via its inhibitory binding to $\mathrm{CDK} 2 /$ cyclin $\mathrm{E}$ and other $\mathrm{CDK} /$ cyclin complexes. ${ }^{60} \mathrm{p} 53$ is a tumor suppressor protein that plays a major role in cellular response to DNA damage and other genomic aberrations. ${ }^{61}$ Activation of p53 can lead to either cell cycle arrest and DNA repair or apoptosis. p53 is phosphorylated at multiple sites and by several different protein kinases. DNA damage induces phosphorylation of p53 at Ser 15 and Ser20 and leads to a reduced interaction between p53 and its negative regulator, mouse double minute 2 homolog. ${ }^{61}$ As shown in Figure 19A and B, the expression level of p21 Waf1/Cip1 was concentration-dependently increased in PC-3 cells when treated with PLB for 24 hours. In comparison to the control cells, there was a 1.7- and 1.9-fold increase in the expression of $\mathrm{p} 21 \mathrm{Waf1} / \mathrm{Cip} 1$ in PC-3 cells treated with PLB at 1 and $5 \mu \mathrm{M}$ for 24 hours, respectively ( $P<0.05$; Figure 19A and B), and the expression level of p27 Kip1 was increased 1.5- and 1.8-fold in DU145 cells treated with PLB at 1 and $5 \mu \mathrm{M}$, respectively. In addition, there was a significant increase (greater than twofold) in the expression level of $21 \mathrm{Waf1} / \mathrm{Cip} 1$ in DU145 cells after treatment with PLB at $5 \mu \mathrm{M}$ for 24 hours $(P<0.05$; Figure $20 \mathrm{~A}$ and B). Moreover, there was a 2.9- and 1.9-fold increase in the expression level of p53 in PC-3 and DU145 cells when treated with $5 \mu \mathrm{M}$ PLB for 24 hours, respectively $(P<0.01$; Figures 19 and 20).

These results demonstrate that PLB can upregulate p21 Waf1/Cip1, p27 Kip1, and p53 in PC-3 and DU145 cells. This will contribute to the cell cycle arrest and apoptosis induced by PLB. Importantly, these results have confirmed the regulatory effect of PLB on cell proliferation-related signaling pathways which was predicted by our bioinformatic study and revealed by our SILAC-based proteomic experiment.

\section{PLB induces apoptosis via mitochondrial pathway and autophagy via modulation of $\mathrm{PI} / 3 \mathrm{~K} / \mathrm{Akt} / \mathrm{mTOR}$ pathway}

Apoptosis and autophagy, two types of predominant programmed cell death, have been found to be potential targets of PLB for its cancer cell killing effect. ${ }^{30} \mathrm{We}$ have observed that PLB significantly induces apoptosis and autophagy in PC-3 and DU145 cells in concentration- and time-dependent manners. The apoptosis and autophagy inducing effects of PLB may be through mitochondrial- and mTOR-mediated pathways. It has been reported that PI3K, mTOR, Akt, and p38MAPK are the upstream regulatory factors of apoptosis and autophagy, and cytochrome $\mathrm{c}$ is a responsive effector to the variations in PI3K/Akt/mTOR and p38MAPK signaling pathways initiating mitochondria-dependent apoptosis. ${ }^{47,62,63}$ Released cytochrome c triggers the activation of caspase family, such as caspase 9 and its downstream caspase 3, and shifting the balance of antiapoptotic to proapoptotic status with the involvement of Bcl-2 family proteins contributes to apoptosis. Inhibition of $\mathrm{PI} 3 \mathrm{~K} / \mathrm{Akt} / \mathrm{mTOR}$ axis can remarkably promote autophagy.

Following the verification of the inhibitory effect of PLB on cell cycle, we further tested the effect of PLB on the expression and phosphorylation of PI3K, mTOR, Akt, p38MAPK, cytochrome c, caspase 9, caspase 3, Bcl-2, and BAX in PC-3 and DU145 cells. Cells were treated with PLB at concentrations of $0.1,1$, and $5 \mu \mathrm{M}$ for 24 hours. There was a significant decrease in the phosphorylation level of PI3K, mTOR, and Akt (Figures 21 and 22) after PC-3 and DU145 cells were treated with PLB. In PC-3 cells with the treatment of PLB at $0.1,1$, and $5 \mu \mathrm{M}$, the phosphorylation level of PI3K decreased 26.6\%, 34.9\%, and 35.5\%, the phosphorylation level of Akt reduced 20.1\%, 28.4\%, and $34.3 \%$, and phosphorylation level of mTOR dropped $12.9 \%, 11.5 \%$, and $31.3 \%$, respectively (Figure 21A and B). Similarly, the phosphorylation level of PI3K reduced $13.4 \%$, $28.1 \%$, and $35.4 \%$, the phosphorylation level of Akt dropped $46.9 \%, 58.7 \%$, and $58.0 \%$, and phosphorylation level of mTOR decreased $26.9 \%, 27.9 \%$, and $36.0 \%$, respectively (Figure 22A and B). Moreover, the phosphorylation of p38MAPK decreased $25.0 \%, 40.0 \%$, and $50.7 \%$ in PC-3 cells (Figure 21A and B) and 37.6\%, 57.4\%, and 63.9\% in DU145 cells (Figure 22A and B) when treated with PLB at $0.1,1$, and $5 \mu \mathrm{M}$, respectively, for 24 hours.

On the other hand, the expression of cytochrome $\mathrm{c}$ was significantly increased in PC-3 and DU145 cells with the treatment of PLB (Figures 21 and 22). Increased release of cytochrome c initiates mitochondria-dependent apoptosis through the sequential activation of caspase family and interruption of the balance of antiapoptotic (Bcl-2) and proapoptotic (BAX) proteins. As shown in Figures 21 and 22, incubation of PC-3 and DU145 cells with PLB significantly increased the cleaved level of caspase 9 and caspase 3 . In PC3 cells, there was 1.6-, 2.1-, and 2.6-fold increase in cleaved level of caspase 9, and 1.3-, 1.3-, and 1.8-fold rise in cleaved level of caspase 3 when treated with PLB at 0.1 , 1 , and $5 \mu \mathrm{M}$, respectively (Figure 21A and B). Similarly, when DU145 cells were treated with PLB at 0.1, 1, and $5 \mu \mathrm{M}$, there was 1.2-, 1.4-, and 1.9-fold increase in cleaved level of caspase 9, and 1.1-, 1.4-, and 2.0-fold elevation in 
A
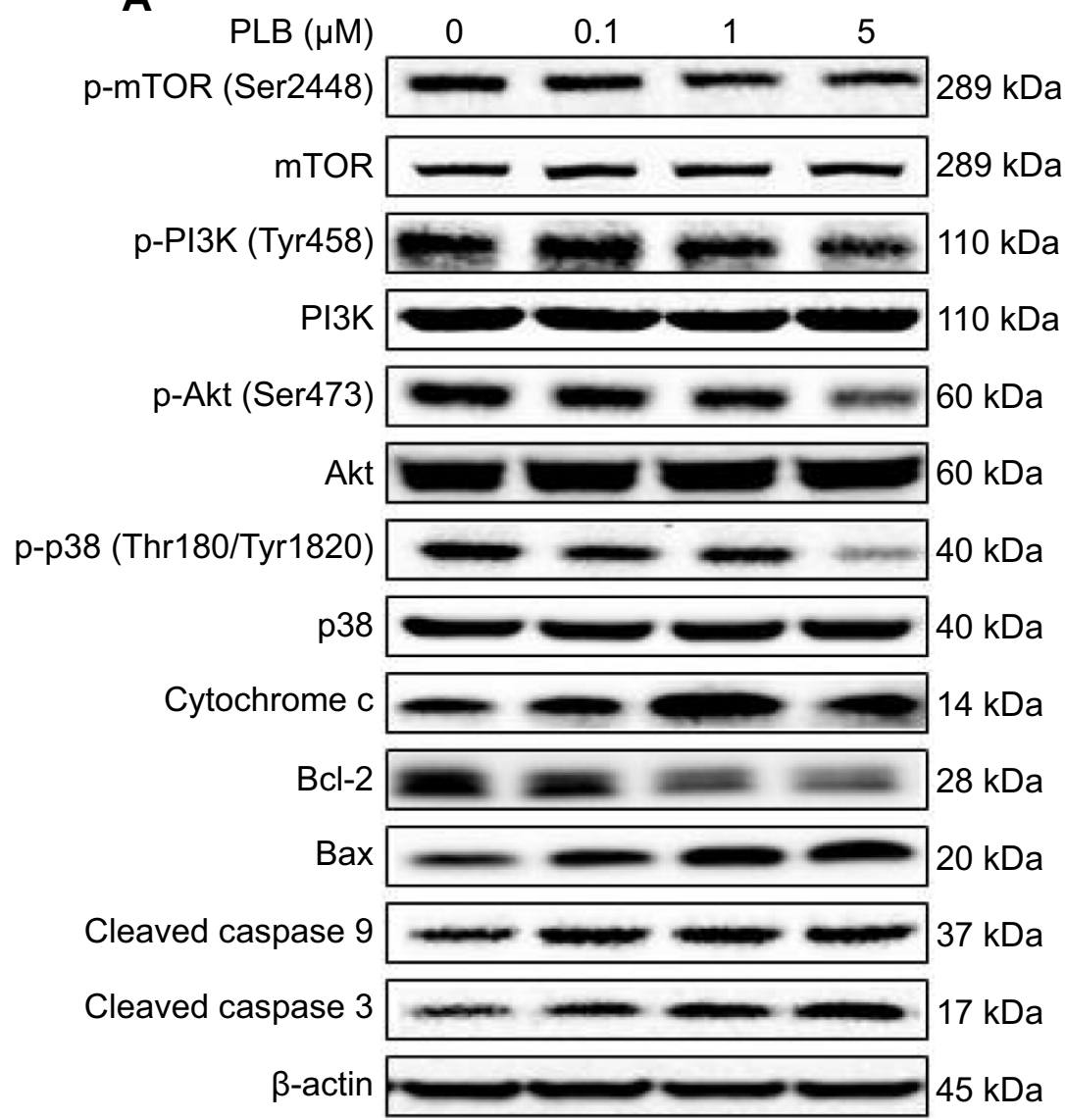

B
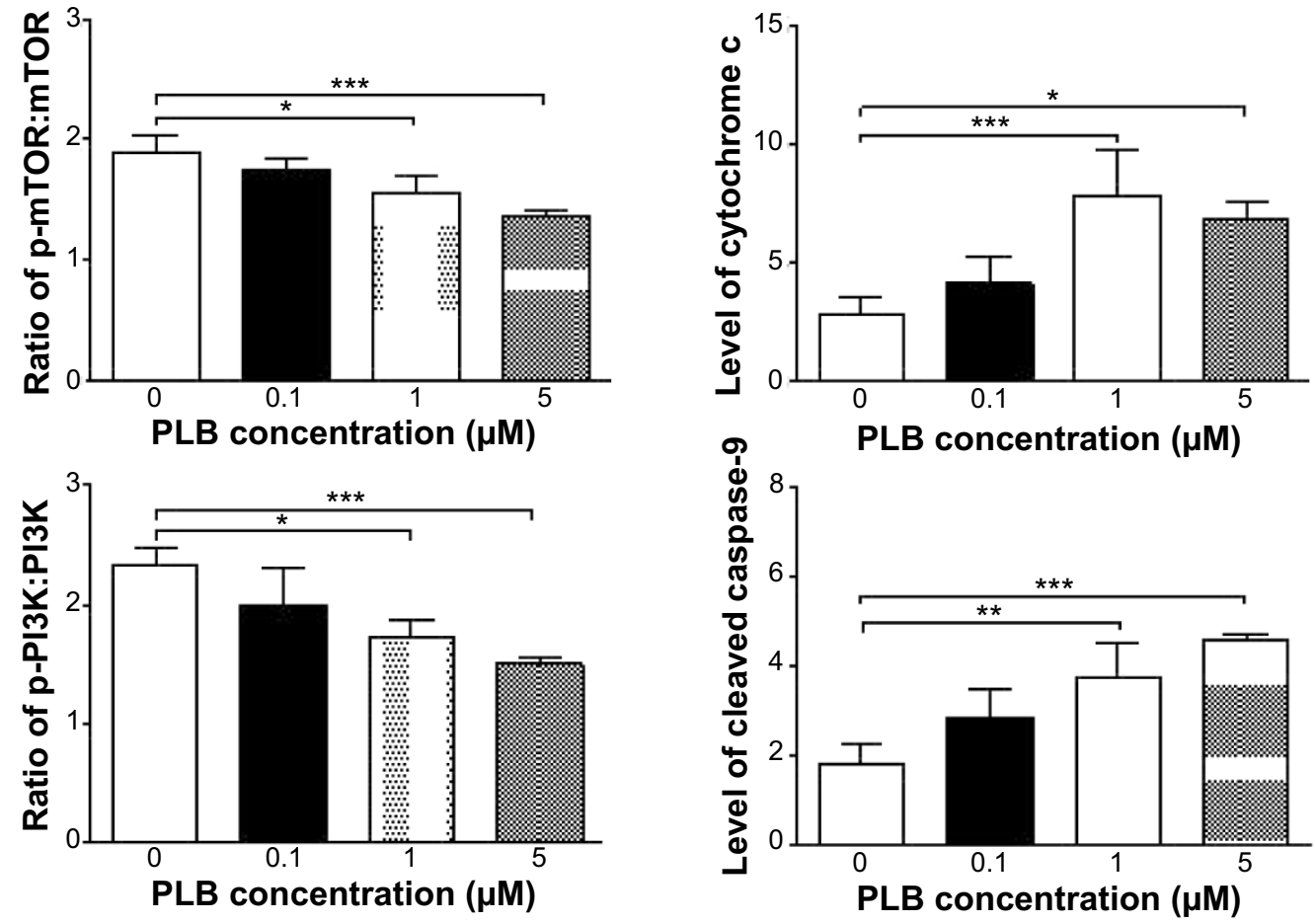

Figure 2 I (Continued) 

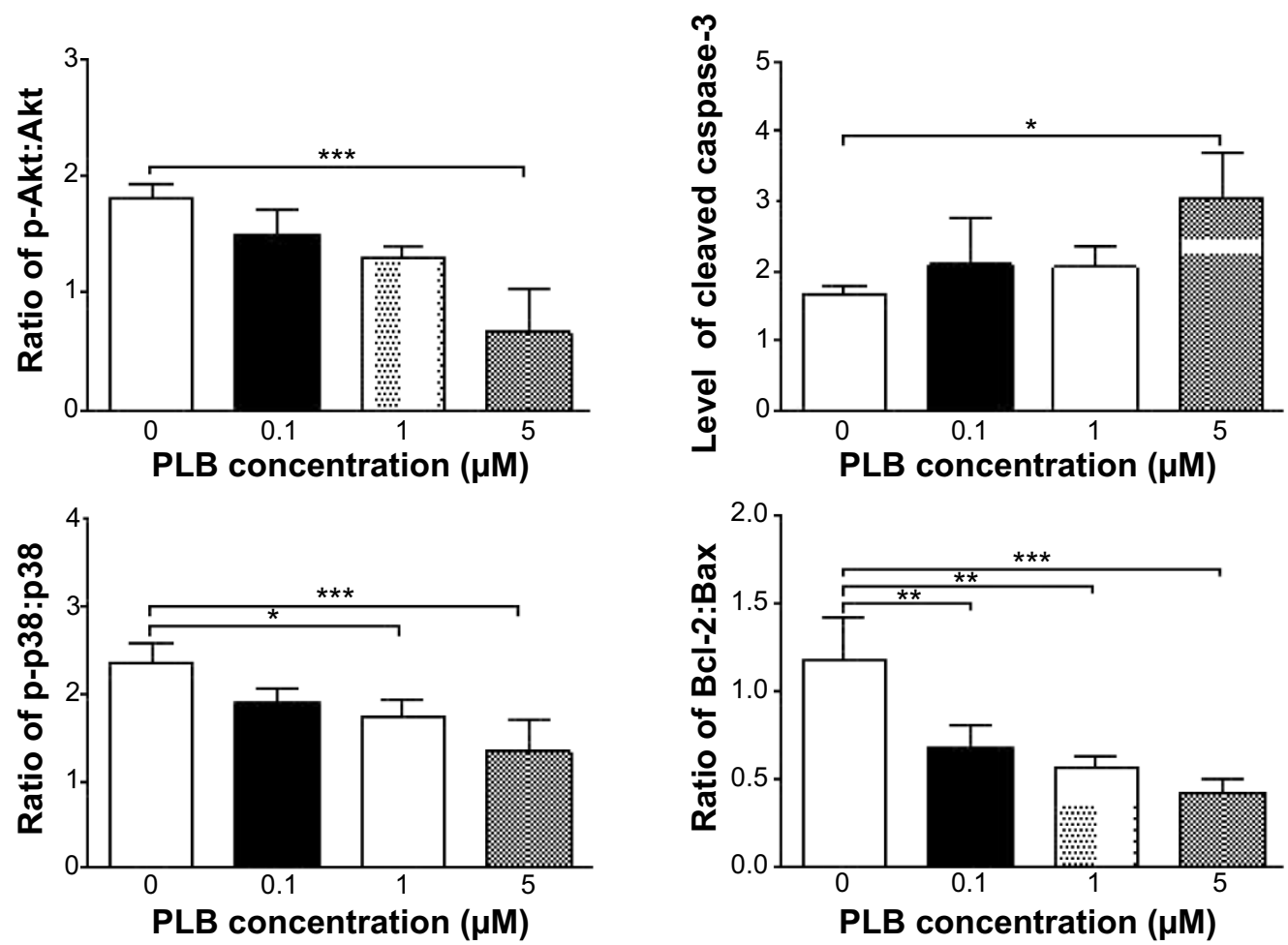

Figure $2 \mathrm{I}$ Effects of PLB treatment on the expression and phosphorylation levels of PI3K, Akt, mTOR, p38MAPK, and cytochrome c in PC-3 cells.

Notes: PC-3 cells were treated with PLB at 0.1 , I, and $5 \mu \mathrm{M}$ for 24 hours and protein samples were subject to Western blot assay. (A) Representative blots of $P$ - and t-PI3K, p- and t-Akt, p- and t-mTOR, p- and t-p38MAPK, and cytochrome c in PC-3 cells, and (B) bar graphs showing the relative levels of p/t-PI3K, p/t-Akt, p/t-mTOR, $\mathrm{p} / \mathrm{tp} 38 \mathrm{MAPK}$, and cytochrome $\mathrm{c}$ in $\mathrm{PC}-3$ cells. Data are the mean \pm standard deviation of three independent experiments. $* P<0.05 ; * * P<0.01$; and $* * * P<0.00 \mathrm{I}$ by one-way analysis of variance.

Abbreviation: PLB, plumbagin.

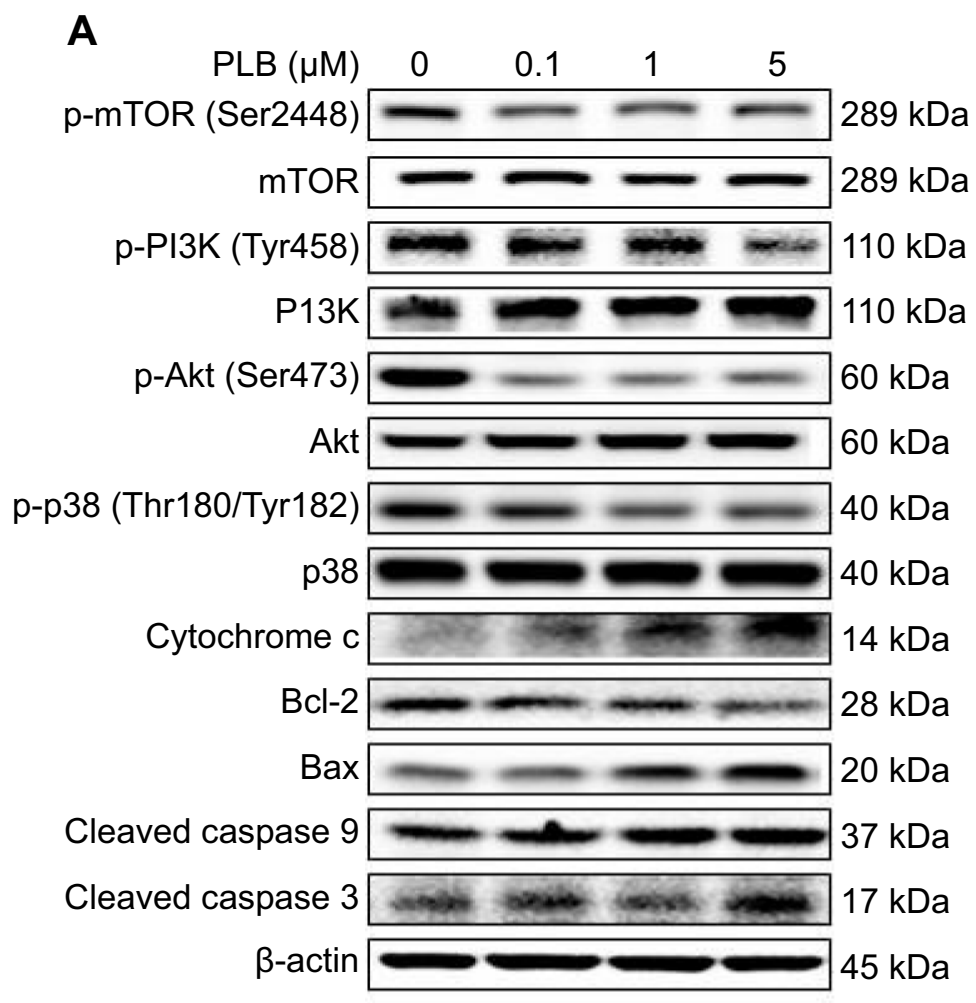

Figure 22 (Continued) 
B
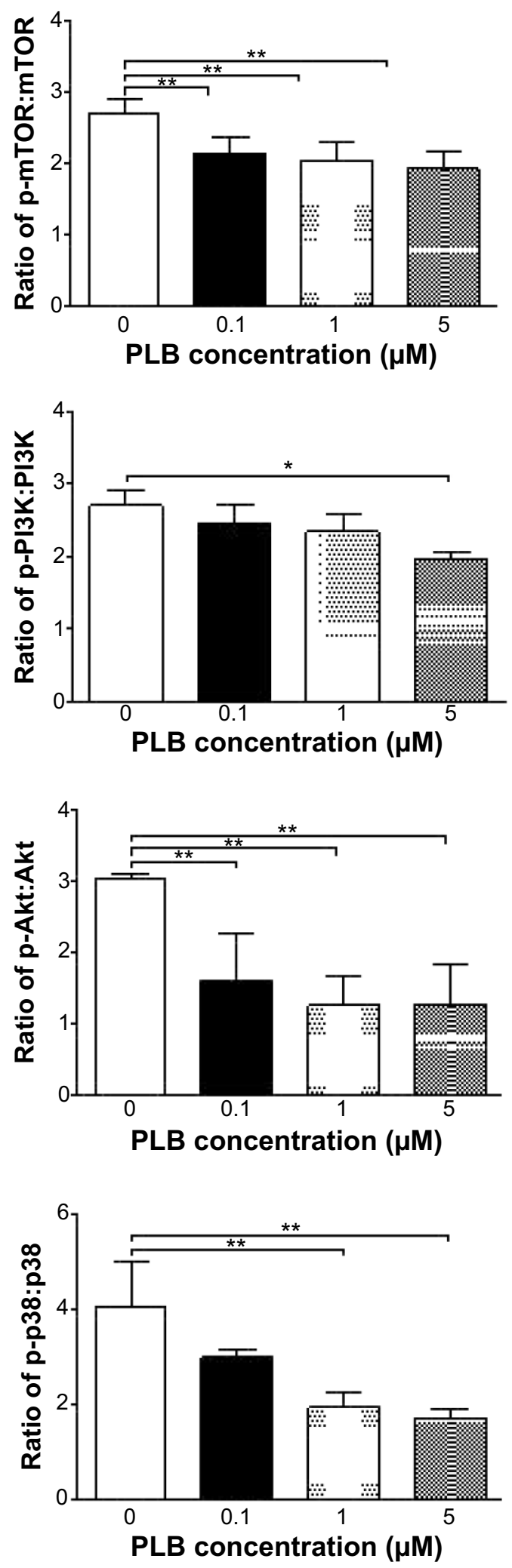
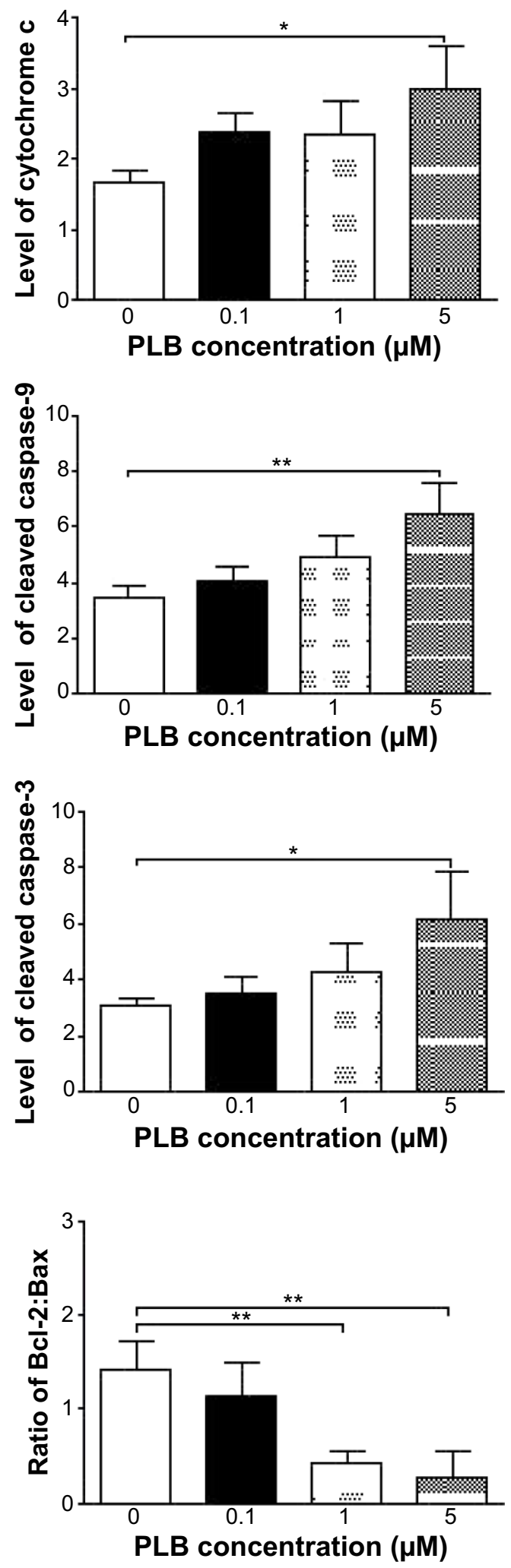

Figure 22 Effects of PLB treatment on the expression and phosphorylation levels of PI3K, Akt, mTOR, p38MAPK, and cytochrome c in DUI45 cells.

Notes: DUI 45 cells were treated with PLB at 0.1 , I, and $5 \mu \mathrm{M}$ for 24 hours and protein samples were subject to Western blot assay. (A) Representative blots of p- and t-PI3K, p- and t-Akt, p- and t-mTOR, p- and t-p38MAPK, and cytochrome $\mathrm{c}$ in DUI45 cells, and (B) bar graphs showing the relative levels of $\mathrm{p} / \mathrm{t}-\mathrm{PI} 3 \mathrm{~K}$, $\mathrm{p} / \mathrm{t}-\mathrm{Akt}$, $\mathrm{p} / \mathrm{t}-\mathrm{mTOR}$, $\mathrm{p} / \mathrm{tp} 38 \mathrm{MAPK}$, and cytochrome $\mathrm{c}$ in DUI45 cells. Data are the mean \pm standard deviation of three independent experiments. $* P<0.05 ; * * P<0.0 \mathrm{I}$ by one-way analysis of variance.

Abbreviation: PLB, plumbagin. 
cleaved level of caspase 3, respectively (Figure 22A and B). Moreover, the ratio of $\mathrm{Bcl} 2$ over $\mathrm{BAX}$ was significantly decreased in both cells treated with PLB. The ratio was decreased $42.4 \%, 52.0 \%$, and $63.7 \%$ in PC-3 cells (Figure $21 \mathrm{~A}$ andB) and $21.2 \%, 70.5 \%$, and $80.9 \%$ in DU145 cells (Figure $22 \mathrm{~A}$ and B) with the treatment of PLB at $0.1,1$, and $5 \mu \mathrm{M}$, respectively. These results clearly showed that PLB induced apoptosis via mitochondrial pathway and autophagy via $\mathrm{PI} 3 \mathrm{~K} / \mathrm{mTOR}$ pathway in PC-3 and DU145 cells, and these data are in agreement with our proteomic findings.

\section{PLB inhibits EMT in PC-3 and DUI 45 cells}

EMT is a critical process involving the initiation, growth, invasion, and metastasis of cancer. ${ }^{20,21,64}$ EMT depends on a reduction in expression of cell adhesion molecules. E-cadherin is considered an active suppressor of invasion and growth of many epithelial cancers. Tight junctions, or zonula occludens, form a continuous barrier to fluids across the epithelium and endothelium..$^{20,21,64}$ They function in regulation of paracellular permeability and in the maintenance of cell polarity, blocking the movement of transmembrane proteins between the apical and the basolateral cell surfaces. Tight junctions are composed of claudin and occludin proteins, which join the junctions to the cytoskeleton. ZO-1, 2, and 3 are peripheral membrane adaptor proteins that link junctional transmembrane proteins such as occludin and claudin to the actin cytoskeleton. ${ }^{20-22,64}$ Cadherins are a superfamily of transmembrane glycoproteins that contain cadherin repeats of approximately 100 residues in their extracellular domain. They mediate calcium-dependent cell-cell adhesion and the classic cadherin subfamily includes N-, P-, R-, B-, and E-cadherins. $^{20,21}$ The cytoplasmic domain of classical cadherins interacts with $\beta$-catenin, $\gamma$-catenin, and p120 catenin. Cancer cells often have upregulated $\mathrm{N}$-cadherin in addition to loss of E-cadherin. ${ }^{20-22}$ Herein, we examined the effect of PLB treatment on EMT-associated markers in PC-3 and DU145 cells using Western blot assay. Incubation of PC-3 cells with PLB resulted in a concentration-dependent increase in the expression level of E-cadherin and decrease in the expression level of N-cadherin (Figure 23A and B). There was a 1.3- and 1.4-fold increase in the expression of E-cadherin when treated with 1 and $5 \mu \mathrm{M}$ PLB for 24 hours, respectively; whereas $5 \mu \mathrm{M}$ PLB suppressed $30.3 \%$ expression level of $\mathrm{N}$-cadherin $(P<0.05$; Figure 23A and B). Consequently, with increasing concentration of PLB, an increased ratio of E-cadherin over N-cadherin was observed. The E-cadherin/N-cadherin ratio was increased from 1.4 at basal level to $1.7,2.4$, and 3.0, when PC-3 cells were treated with $0.1,1$ and $5 \mu \mathrm{M}$ PLB for 24 hours, respectively ( $P<0.05$; Figure 23A and B). In DU145 cells, there was a 1.6- and 1.5-fold increase in the expression of E-cadherin when cells were treated with 1 and 5 $\mu \mathrm{M}$ PLB, respectively (Figure 24A and B). Meanwhile, PLB decreased the expression of $\mathrm{N}$-cadherin, but no significant effect was observed. However, the E-cadherin/N-cadherin ratio was increased from 1.1 to $1.4,1.9$, and 2.0, when DU145 cells were treated with $0.1,1$, and $5 \mu \mathrm{M}$ PLB, respectively $(P<0.05$; Figure 24A and B).

In order to further examine the effect of PLB on EMT in PC-3 and DU145 cells, we measured the expression levels of several key regulators of E-cadherin. Snail and slug (both zinc finger transcriptional factors) together with TCF8/ZEB1 are suppressors of E-cadherin in EMT. ${ }^{20,21}$ In addition, snail blocks the cell cycle and confers resistance to cell death, and slug protects damaged cells from apoptosis by repressing p53-induced transcription of the proapoptotic Bcl-2 family protein PUMA. ${ }^{20,21}$ PLB significantly reduced the expression level of snail and slug in both cell lines (Figures 23 and 24). In PC-3 cells, $5 \mu \mathrm{M}$ PLB significantly suppressed the expression level of snail by $19.6 \%, 30.8 \%$, and $35.4 \%$, and of slug by $29.2 \%, 40.0 \%$, and $37.6 \%$ when treated with $0.1,1$, and $5 \mu \mathrm{M}$ PLB for 24 hours, respectively $(P<0.01$; Figure $23 \mathrm{~A}$ and B). In DU145 cells, 1 and $5 \mu \mathrm{M}$ PLB significantly suppressed the expression level of snail by $21.8 \%$ and $28.9 \%$, respectively. Treatment of cells with $5 \mu \mathrm{M}$ PLB for 24 hours significantly reduced the expression level of slug by $38.1 \%$ $(P<0.05$; Figure 24A and B). Furthermore, PLB induced a concentration-dependent reduction in the expression level of TCF-8/ZEB1 in PC-3 and DU145 cells. In PC-3 cells, 1 and $5 \mu \mathrm{M}$ PLB significantly suppressed the expression level of TCF-8/ZEB1 by $36.2 \%$ and $51.7 \%$, respectively (Figure $23 \mathrm{~A}$ and B). Similarly, there was a $57.5 \%$ reduction in the expression of TCF-8/ZEB1 in DU145 cells treated with $5 \mu \mathrm{M}$ of PLB $(P<0.001$; Figure 24A and B).

Vimentin is a type III intermediate filament protein that is expressed in mesenchymal cells. ${ }^{20-22,64} \beta$-catenin can act as an integral component of a protein complex in adherent junctions that helps cells maintain epithelial layers, and $\beta$-catenin participates in the Wnt signaling pathway as a downstream target. ${ }^{22,64}$ Treatment of cells with $5 \mu \mathrm{M}$ PLB significantly suppressed the expression level of vimentin by $36.0 \%$ in PC-3 cells $(P<0.05$; Figure $23 \mathrm{~A}$ and B). PLB at 0.1 and $1 \mu \mathrm{M}$ reduced vimentin level by $23.8 \%-26.4 \%$, but did not achieve statistical significance. In DU145 cells, treatment with PLB at $0.1,1$, and $5 \mu \mathrm{M}$ for 24 hours resulted in a $10.0 \%, 19.3 \%$, and $29.7 \%$ reduction in vimentin expression levels, respectively $(P<0.05-0.001$; Figure 24A and B). 
A

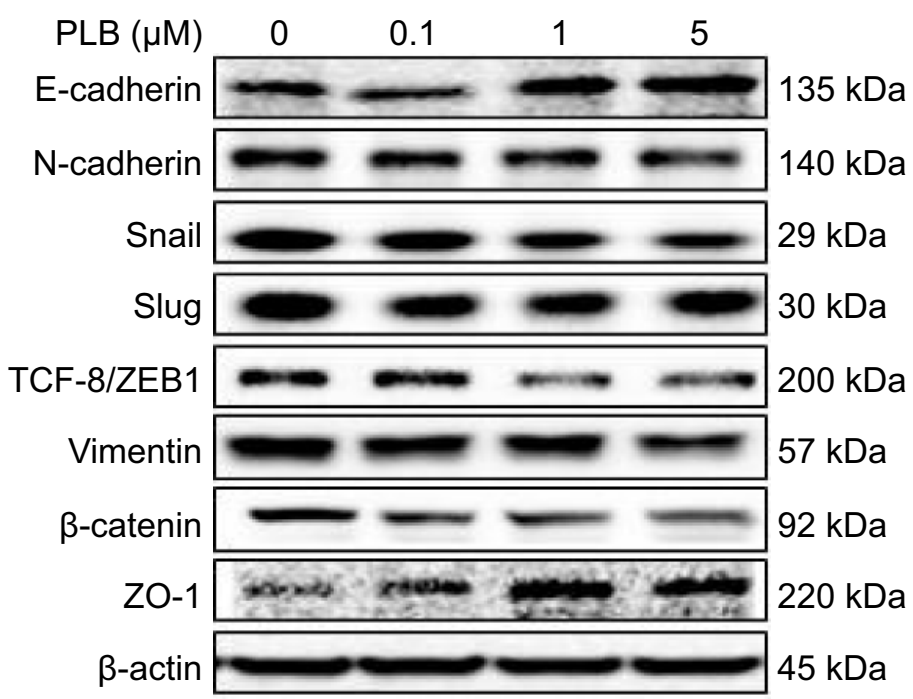

B
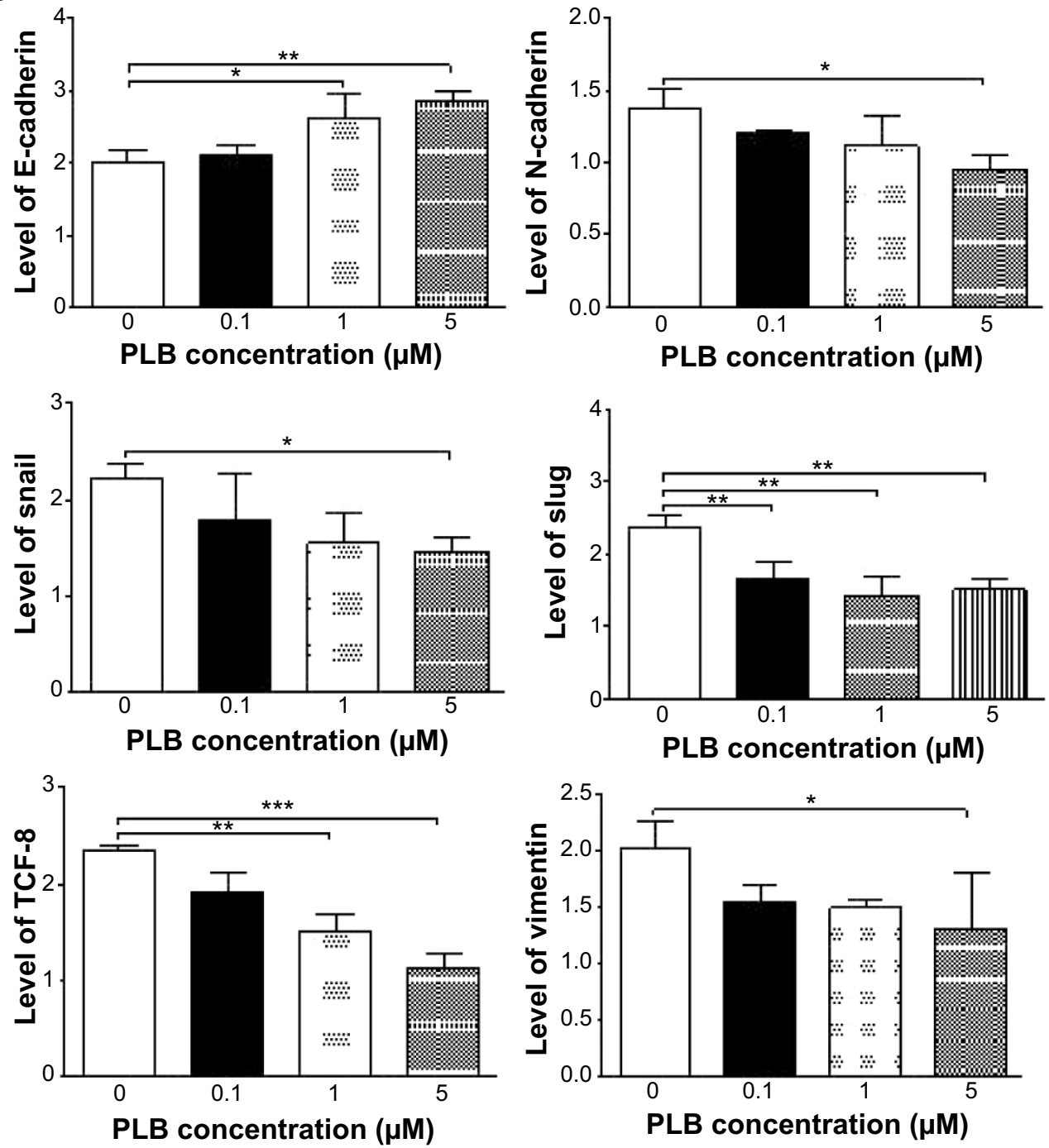

Figure 23 (Continued) 

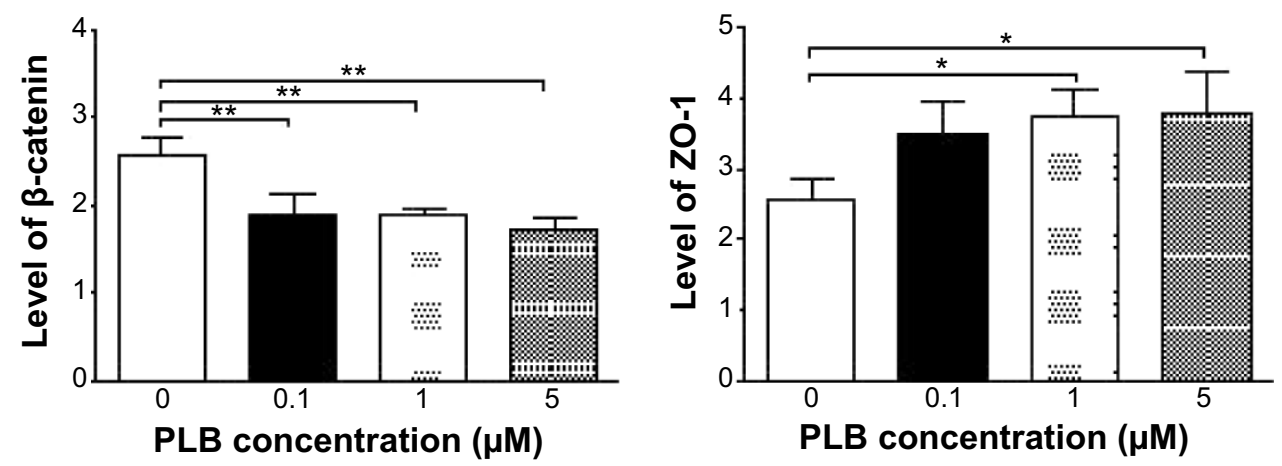

Figure 23 Dose effect of PLB on the expression level of selected EMT markers in PC-3 cells.

Notes: PC-3 cells were treated with PLB at 0.1 , I, and $5 \mu$ M for 24 hours and protein samples were subject to Western blot assay. (A) Representative blots of E-cadherin, $\mathrm{N}$-cadherin, snail, slug, TCF-8/ZEBI, vimentin, $\beta$-catenin, ZO-I, and $\beta$-actin in PC-3 cells treated with PLB at $0.1, I$, and $5 \mu$ M for 24 hours, and (B) bar graphs showing the levels of E-cadherin, $\mathrm{N}$-cadherin, snail, slug, TCF-8/ZEBI, vimentin, $\beta$-catenin, and ZO-I in PC-3 cells. Data represent the mean \pm standard deviation of three independent experiments. $* P<0.05 ; * * P<0.01 ; * * * P<0.00$ I by one-way analysis of variance.

Abbreviations: EMT, epithelial-mesenchymal transition; PLB, plumbagin.

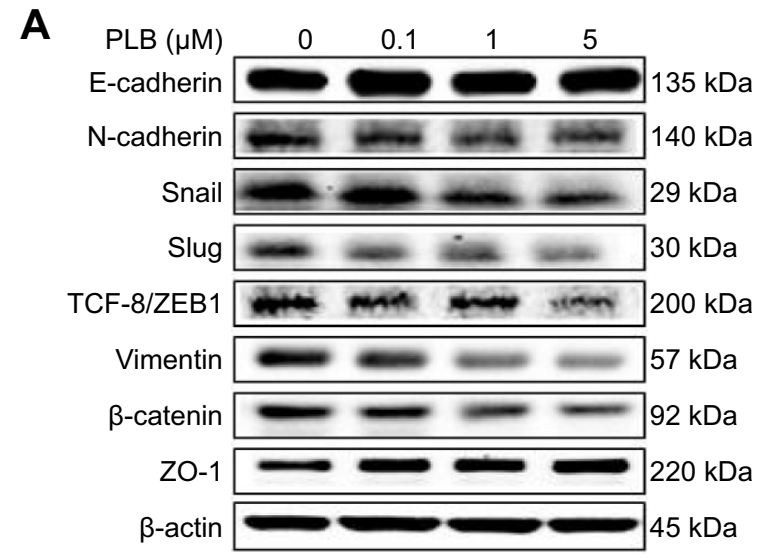

B
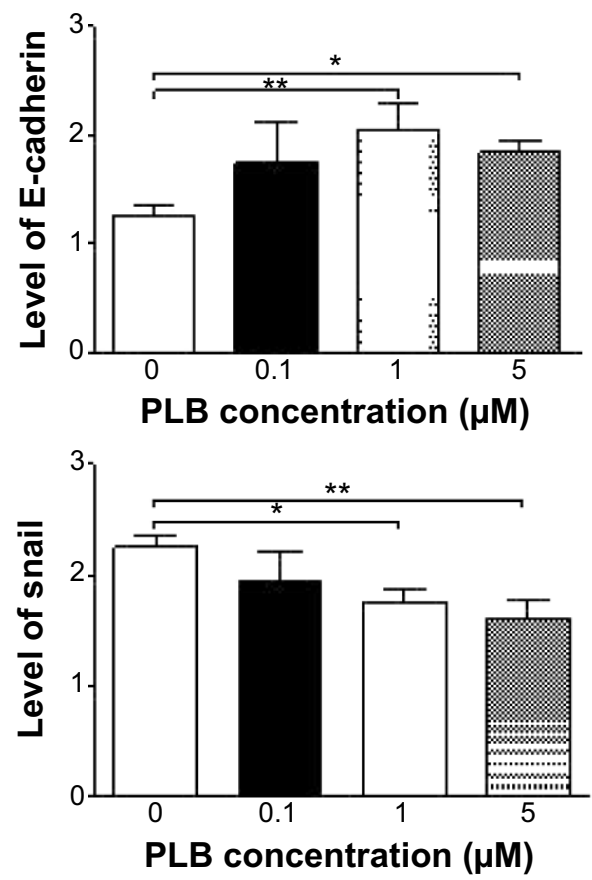
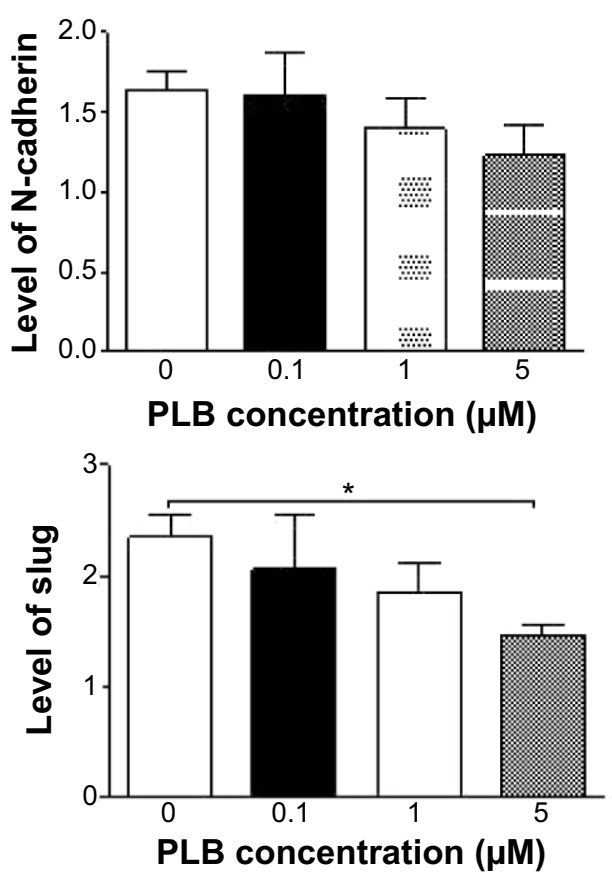

Figure 24 (Continued) 

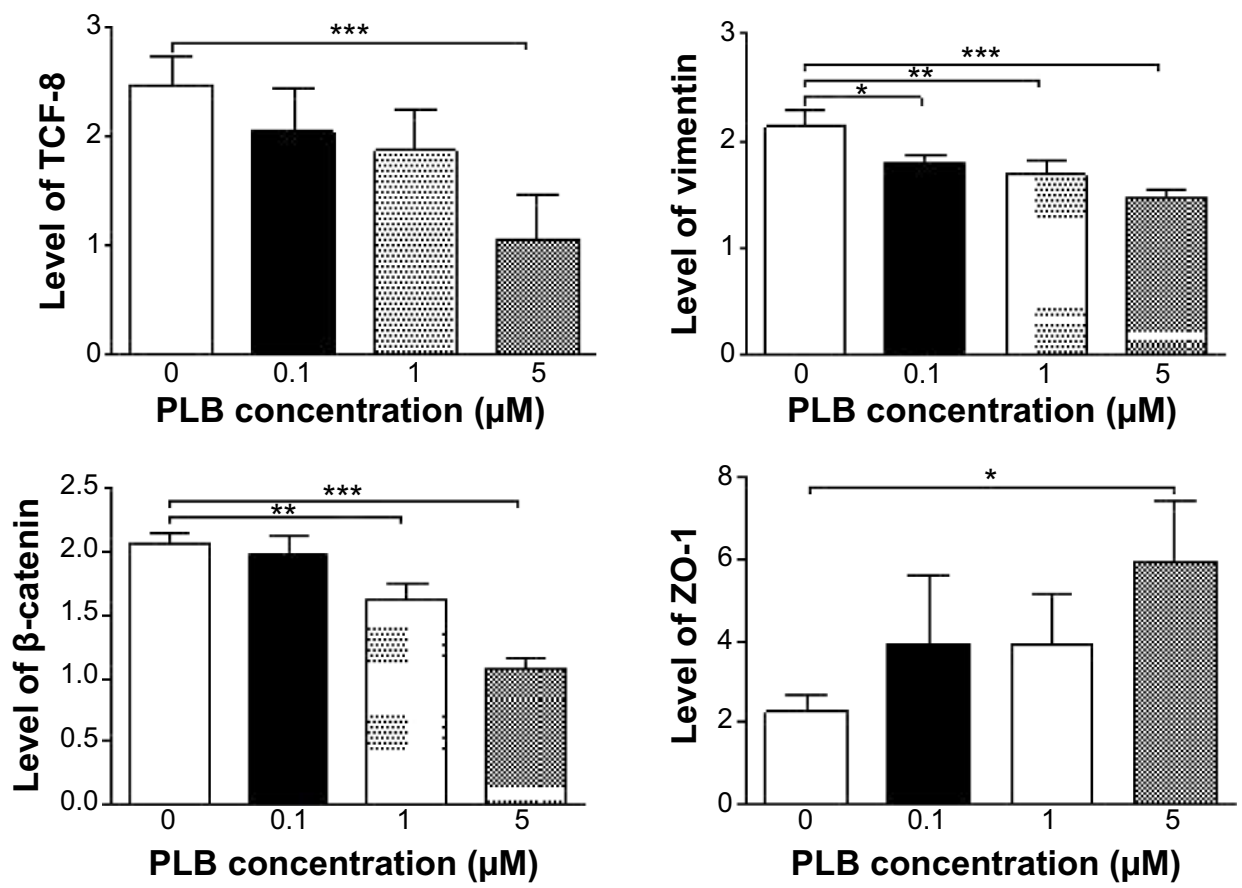

Figure 24 Dose-effect of PLB on the expression level of selected EMT markers in DUI45 cells.

Notes: DUI45 cells were treated with PLB at 0.1 , I, and $5 \mu \mathrm{M}$ for 24 hours and protein samples were subject to Western blot assay. (A) Representative blots of E-cadherin, $\mathrm{N}$-cadherin, snail, slug, TCF-8/ZEBI, vimentin, $\beta$-catenin, ZO-I, and $\beta$-actin in DUI 45 cells treated with PLB at 0.1 , I, and $5 \mu$ M for 24 hours, and (B) bar graphs showing the levels of E-cadherin, $\mathrm{N}$-cadherin, snail, slug, TCF-8/ZEBI, vimentin, $\beta$-catenin, and ZO-I in DUI45 cells. Data represent the mean \pm standard deviation of three independent experiments. $* P<0.05 ; * * P<0.0$ I; $* * * P<0.00$ I by one-way analysis of variance.

Abbreviations: EMT, epithelial-mesenchymal transition; PLB, plumbagin.

There was a significant reduction in the expression level of $\beta$-catenin in both cell lines treated with PLB at $0.1,1$, and $5 \mu \mathrm{M}$ for 24 hours. PLB at $0.1,1$, and $5 \mu \mathrm{M}$ significantly decreased the expression level of $\beta$-catenin by $25.7 \%$, $26.2 \%$, and $32.6 \%$ in PC-3 cells, respectively (Figure 23A and $\mathrm{B}$ ), and 1 and $5 \mu \mathrm{M}$ PLB significantly reduced $\beta$-catenin expression by $21.0 \%$ and $47.5 \%$ in DU145 cells, respectively (Figure 24A and B).

Furthermore, we examined the time course of the effect of PLB on the expression of selected EMT markers in PC-3 and DU145 cells over 48 hours. There was a significant inhibitory effect of PLB on EMT in both cells (Figures 25 and 26). In comparison to the control cells, treatment of PC-3 cells with $5 \mu \mathrm{M}$ PLB significantly increased the expression of E-cadherin by 1.7- and 2.4-fold, while the expression of $\mathrm{N}$-cadherin was decreased by $49.2 \%$ and $58.1 \%$ after 24 and 48 hours, respectively, which in turn led to a significant increase in the ratio of E-cadherin over $\mathrm{N}$-cadherin. The expression of vimentin was significantly decreased by $40.0 \%$ and $51.4 \%$ with the $5 \mu \mathrm{M}$ PLB treatment for 24 and 48 hours, respectively. Moreover, the expression of $\beta$-catenin was reduced by $4.06 \%$ and $41.7 \%$ with the $5 \mu \mathrm{M}$ PLB treatment for 24 and 48 hours, respectively (Figure 25A and B). In DU145 cells, incubation with
$5 \mu \mathrm{M}$ PLB for 24 and 48 hours led to a 2.0- and 2.3-fold increase in the expression of E-cadherin, respectively, and resulted in a $38.8 \%$ and $45.3 \%$ reduction in the expression of $\mathrm{N}$-cadherin compared to the control cells, respectively. Consequently, it led to an increase in the ratio of E-cadherin over N-cadherin. Moreover, treatment of DU145 cells with $5 \mu \mathrm{M}$ of PLB induced a time-dependent decrease in the expression of $\beta$-catenin and vimentin by $42.8 \%$ and $48.6 \%$, and $30.9 \%$ and $40.8 \%$, to 24 hour and 48 hour treatment, respectively (Figure 26A and $\mathrm{B}$ ).

Finally, the expression of ZO-1 was examined in PC-3 and DU145 cells exposed to PLB. ZO-1 and -2 are required for tight junction formation and function. In subconfluent proliferating cells, ZO-1 and ZO-2 have been shown to colocalize to the nucleus and play a role in transcriptional regulation, possibly through facilitating nuclear import/export of transcriptional regulators. ${ }^{18,46}$ There was a significant effect of PLB on the expression of ZO-1 observed in both cell lines (Figures 23 and 24). Treatment of PC-3 cells with 1 and $5 \mu \mathrm{M}$ PLB for 24 hours resulted in a 1.5-fold increase in ZO-1 expression and $5 \mu \mathrm{M}$ PLB resulted in a 2.6-fold increase in the expression level of ZO-1 in DU145 cells $(P<0.05$; Figures 23 and 24). These results from Western blot assay are consistent with our proteomic data. 


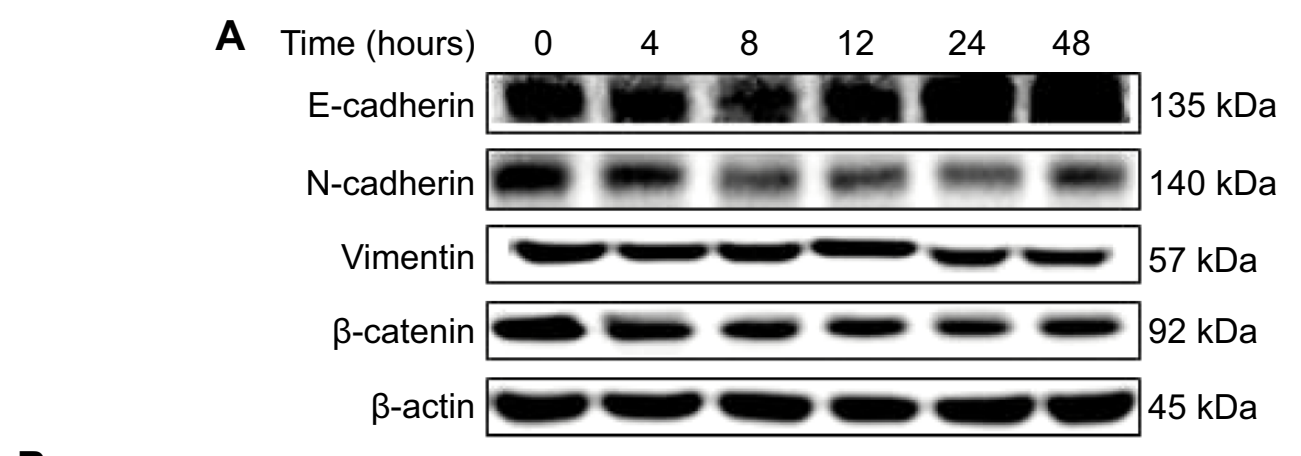

B
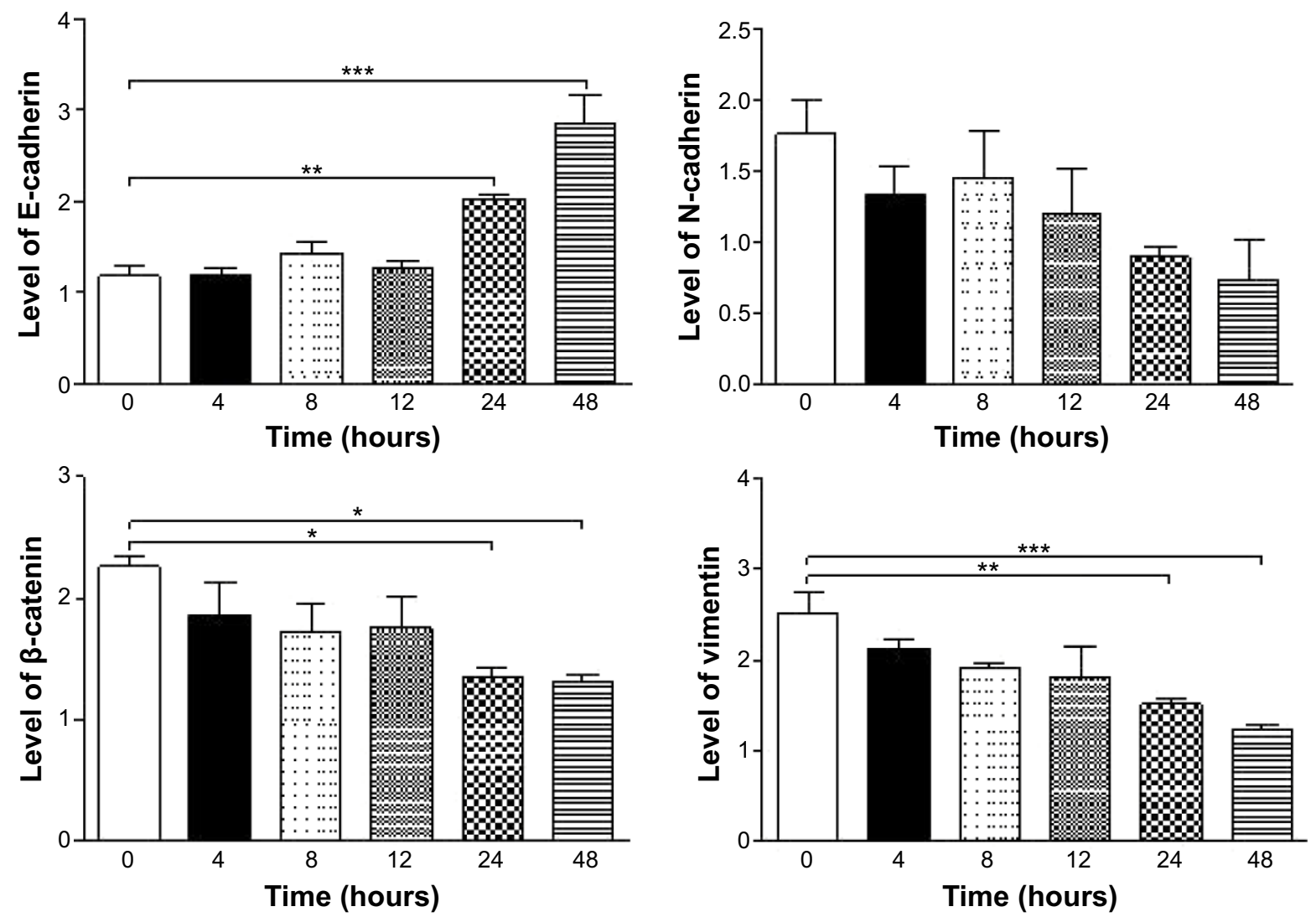

Figure 25 Effects of PLB on the expression level of selected EMT markers in PC-3 cells over 48 hours.

Notes: PC-3 cells were treated with $5 \mu$ M PLB over 48 hours and protein samples were subject to Western blot assay. (A) Representative blots of E-cadherin, N-cadherin, vimentin, $\beta$-catenin, and $\beta$-actin in PC-3 cells, and (B) bar graphs showing the levels of E-cadherin, $\mathrm{N}$-cadherin, vimentin, and $\beta$-catenin in PC-3 cells. Data represent the mean \pm standard deviation of three independent experiments. $* P<0.05 ; * * P<0.01 ; * * * P<0.00$ I by one-way analysis of variance.

Abbreviations: EMT, epithelial-mesenchymal transition; PLB, plumbagin.

\section{PLB regulates EMT via Sirt I-mediated pathway} in PC-3 and DUI45 cells

Sirt1 plays an important role in the regulation of EMT and our proteomic data suggest that PLB may regulate Sirt1mediated signaling pathways. Thus, we speculated that PLB may regulate Sirt 1 expression in PC-3 and DU145 cells. We examined the effect of PLB on the expression of Sirt1 in both cell lines and evaluated the effect of STL (an inhibitor of Sirt ${ }^{65}$ ) on the expression of E-cadherin and N-cadherin in PC-3 and DU145 cells. As shown in Figure 27A and B, incubation of PC-3 and DU145 cells with PLB at $0.1,1$, and $5 \mu \mathrm{M}$ resulted in a significant decrease in the expression of Sirt1. There was a $32.4 \%$ reduction in the expression level of Sirt 1 when PC-3 cells were treated with $5 \mu \mathrm{M}$ PLB (Figure 27A and $\mathrm{B}$ ), and a $38.1 \%, 44.6 \%$, and $56.1 \%$ decrease in the expression level of Sirt1 in DU145 cells treated with 0.1, 1 , and $5 \mu \mathrm{M}$ PLB, respectively (Figure 27A and B). Treatment of PC-3 cells with $25 \mu \mathrm{M}$ STL alone significantly increased the expression level of E-cadherin by $111.1 \%$ and decreased the level of $\mathrm{N}$-cadherin by $46.2 \%$ compared 


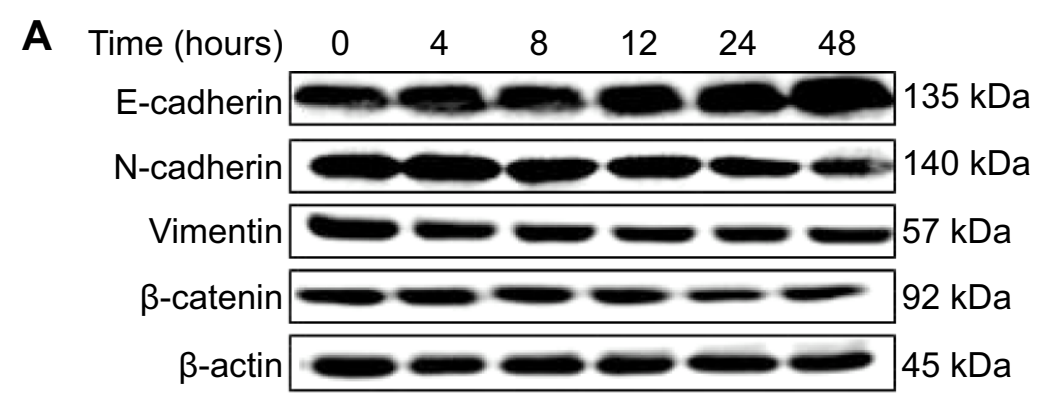

B
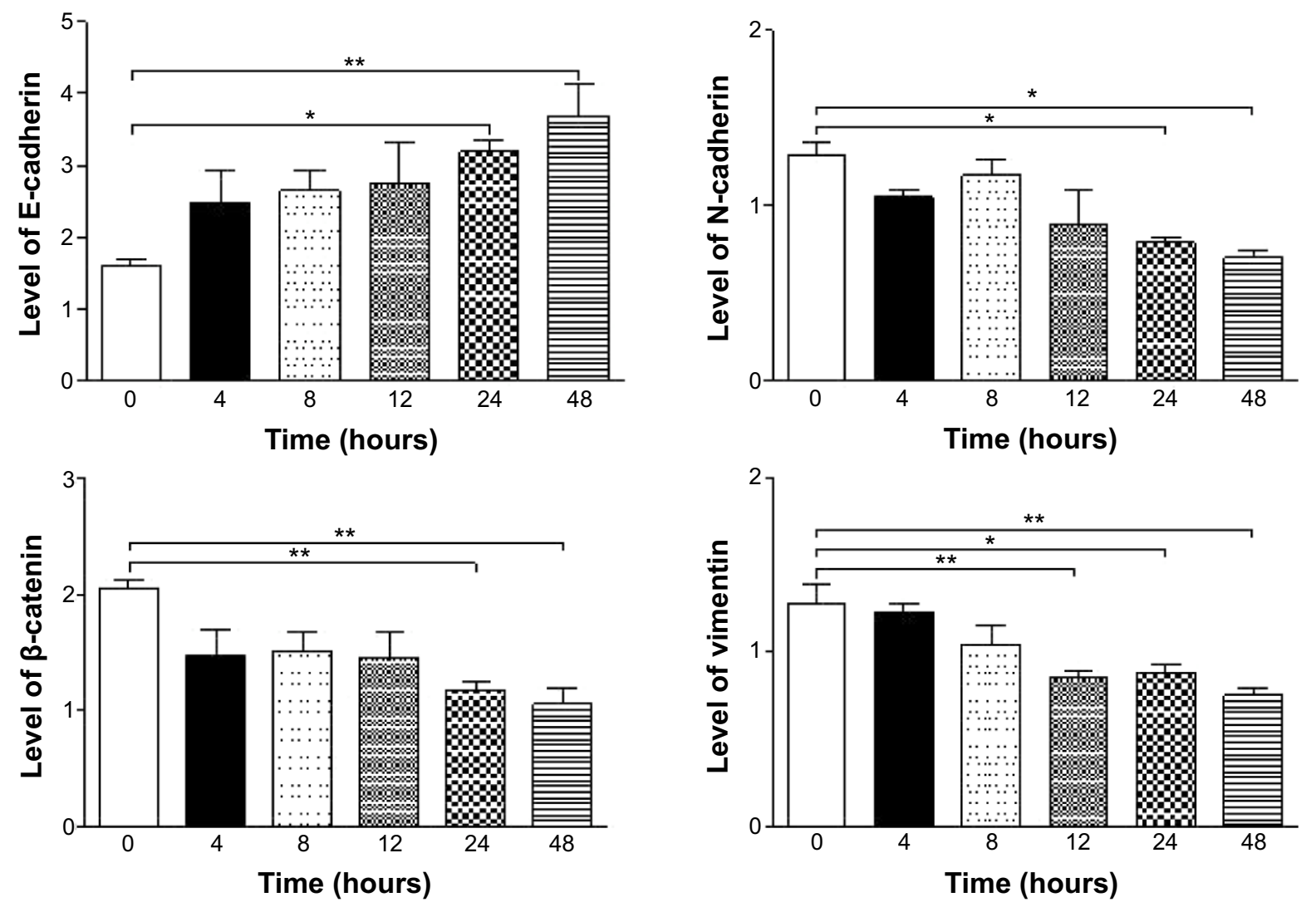

Figure 26 Effects of PLB on the expression level of selected EMT markers in DUI 45 cells over 48 hours.

Notes: DUI 45 cells were treated with $5 \mu$ M PLB over 48 hours and protein samples were subject to Western blot assay. (A) Representative blots of E-cadherin, N-cadherin, vimentin, $\beta$-catenin, and $\beta$-actin in DUI45 cells, and (B) bar graphs showing the levels of E-cadherin, N-cadherin, vimentin, and $\beta$-catenin in DUI45 cells. Data represent the mean \pm standard deviation of three independent experiments. $* P<0.05 ; * * P<0.0$ l by one-way analysis of variance.

Abbreviations: EMT, epithelial-mesenchymal transition; PLB, plumbagin.

to vehicle-treated cells $(P<0.05$; Figure $27 \mathrm{C}$ and D), resulting in a significantly increased ratio of E-cadherin/Ncadherin (3.9 versus 1.0). Addition of $25 \mu \mathrm{M}$ STL caused a $45.3 \%$ increase in PLB-induced expression of E-cadherin $(P<0.05)$ while only slightly decreasing the expression level of N-cadherin (by 28.4\%) in PC-3 cells compared to cells treated with $5 \mu \mathrm{M}$ PLB, resulting in a significantly increased E-cadherin/ $\mathrm{N}$-cadherin ratio (4.8 versus 2.3; $P<0.05$; Figure 27C and D). The downregulation of Sirt1 by PLB may partially contribute to its autophagy-inducing and EMT-inhibitory effects.
In DU145 cells, STL alone induced a 1.5-fold increase in the expression level of E-cadherin and reduced the level of $\mathrm{N}$-cadherin by $25.3 \%$ compared to vehicle-treated cells, resulting in a significantly increased ratio of E-cadherin/ $\mathrm{N}$-cadherin (1.2 versus $2.4 ; P<0.05$; Figure $27 \mathrm{C}$ and $\mathrm{D}$ ). Incubation of STL together with $5 \mu \mathrm{M}$ PLB only slightly decreased the expression level of E-cadherin (by $9.7 \%$ ) but significantly decreased the expression level of $\mathrm{N}$-cadherin by $16.8 \%$ compared to PLB-treated cells, resulting in an insignificantly changed E-cadherin/N-cadherin ratio (Figure 27C and D). These results indicate that inhibition of Sirt1 blocks EMT by restoring the 


\section{A}

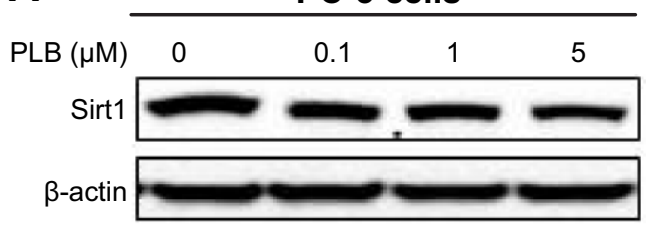

B

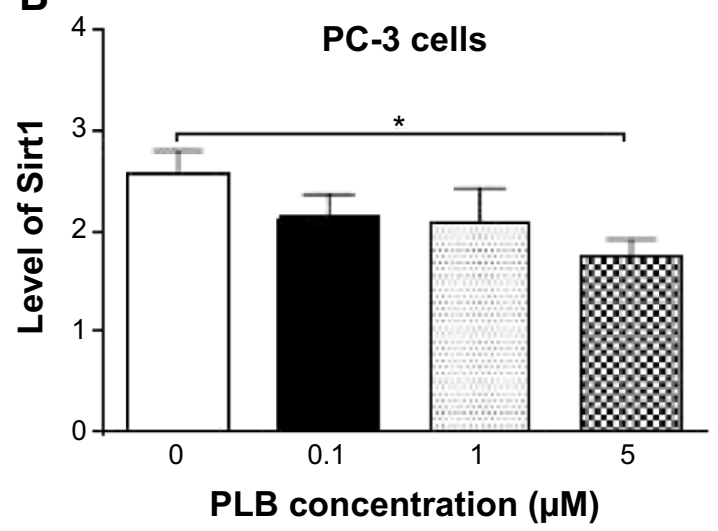

C

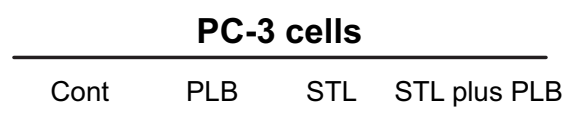

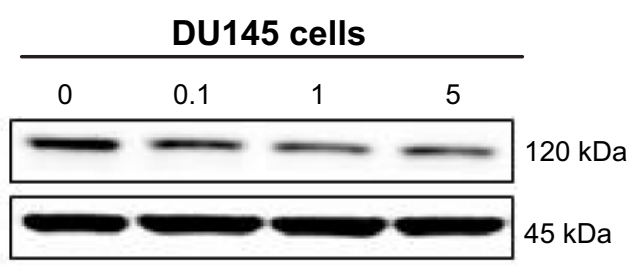
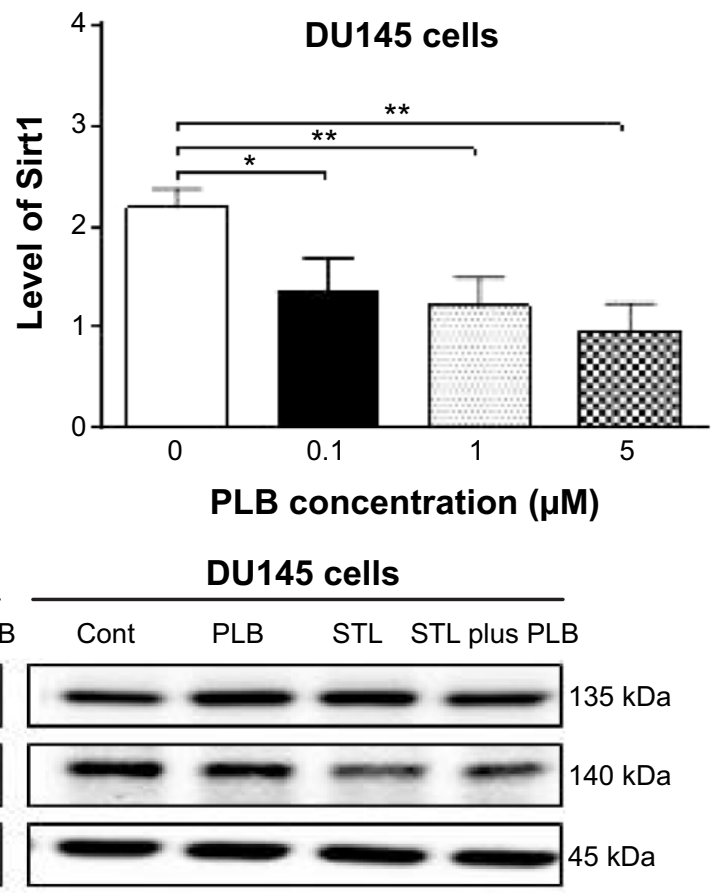

D
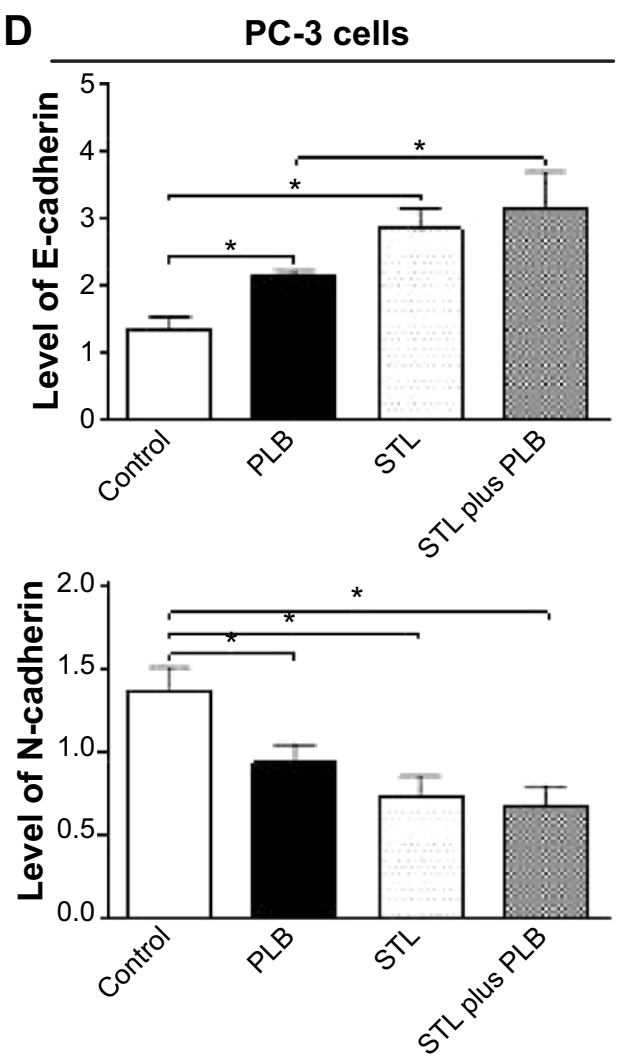

DU145 cells
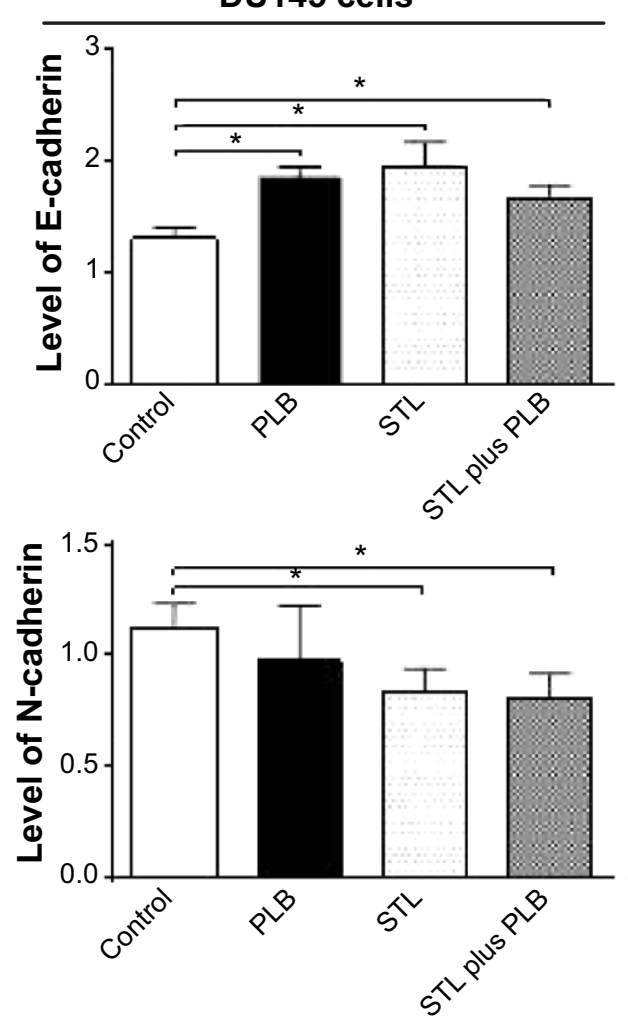

Figure 27 (Continued) 

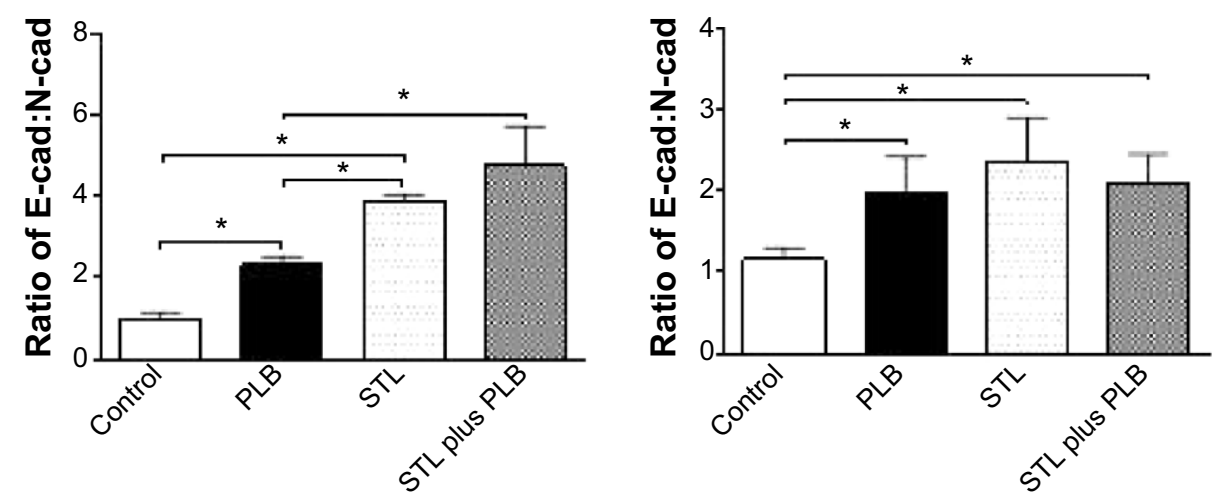

Figure 27 The role of Sirt-I in PLB-induced EMT inhibition in PC-3 and DUI45 cells.

Notes: Cells were treated with PLB at $0.1, I$, and $5 \mu$ M for 24 hours and protein samples were subject to Western blot assay. (A) Representative blots of Sirt I and $\beta$-actin in PC-3 and DUI45 cells; (B) bar graphs showing the relative expression level of Sirt-I in PC-3 and DUI 45 cells; (C) representative blots of E-cadherin, N-cadherin, and $\beta$-actin in PC-3 and DUI45 cells; and (D) bar graphs showing the relative expression level of E-cadherin and N-cadherin in PC-3 and DUI45 cells. Data are the mean \pm standard deviation of three independent experiments. $* P<0.05$; $* * P<0.0$ I by one-way analysis of variance.

Abbreviations: EMT, epithelial-mesenchymal transition; PLB, plumbagin; STL, sirtinol.

E-cadherin and N-cadherin balance, and that inhibition of Sirt1 augments the inhibitory effect of PLB on EMT in PC-3 cells, but not in DU145 cells. The data from Western blot assay support our findings from our quantitative proteomic study where differences in the proteomic responses to PLB treatment were observed between PC-3 and DU145 cells.

\section{PLB modulates ROS and redox pathways in PC-3 and DUI45 cells}

Increased intracellular ROS level can cause cell death through various mechanisms, including mitochondria-mediated apoptosis and modulation of autophagy. ${ }^{66-68}$ Following the observation and verification of proapoptotic effect of PLB in PC-3 and DU145 cells, we examined the effect of PLB on ROS production in both cell lines. Cells were treated with PLB at $0.1,1$, and $5 \mu \mathrm{M}$ for 24 hours. The intracellular level of ROS was significantly increased by 1.9 -fold in PC-3 cells treated with $5 \mu \mathrm{M}$ PLB (Figure 28A); in DU145 cells, there was 1.1-, 1.3-, and 1.2-fold elevation in the intracellular level of ROS when cells were treated with PLB at $0.1,1$, and $5 \mu \mathrm{M}$, respectively (Figure 28B). Apo, an NADPH oxidase inhibitor, significantly suppressed the PLB-induced ROS production in both cell lines $(P<0.05$; Figure 28A and B). Moreover, there was a significant increase in the intracellular ROS level when cells were treated over 72 hours. After incubation of PC-3 and DU145 cells with $5 \mu \mathrm{M}$ PLB for 72 hours, there was a 1.4- and 1.9-fold increase in the intracellular level of ROS, respectively (Figure 28C and D). The ROS-inducing effect of PLB in PC-3 and DU145 cells reveals that PLB induces the generation of ROS in many types of cancer cells, and this may be the shared key mechanism for the anticancer effects of PLB on these types of cancer cells.
The data from Western blot assay further confirms our main finding in SILAC-based quantitative proteomic study where ROS-related pathways were regulated by PLB in both PC-3 and DU145 cells.

\section{Discussion}

Treatment of advanced prostate cancer remains a major challenge because of poor efficacy of current therapies and chemotherapy. There is an increased interest in seeking new effective drugs for prostate cancer from natural compounds. PLB has been found to exhibit anticancer activities for prostate cancer in vitro and in vivo, which are attributed to its effects on multiple signaling pathways related to cell cycle arrest, apoptosis, autophagy, EMT, and redox homeostasis. ${ }^{24-28,30,31,46,69,70}$ In the present study, we compared the global proteomic responses to PLB treatment with regard to cell cycle, programmed cell death, EMT and related molecular targets, and signaling pathways in PC-3 and DU145 cells. The quantitative proteomic study showed that a large number of important proteins regulate cell proliferation, growth, cell death, and migration in both PC-3 and DU145 cells. Importantly, the proteomic analysis showed remarkable differences in the responses to PLB treatment between PC-3 and DU145 cells. Such differences are largely validated by our Western blot analysis, although we could not identify the reasons for such significant differences observed with the two commonly used human prostate cancer cell lines.

Before conducting SILAC-based quantitative proteomic study, we performed a bioinformatic analysis to predict the potential targets of PLB using an established approach, and we have found that PLB might interact with 78 proteins including those involved in cell proliferation and apoptosis; nucleic acid 
A

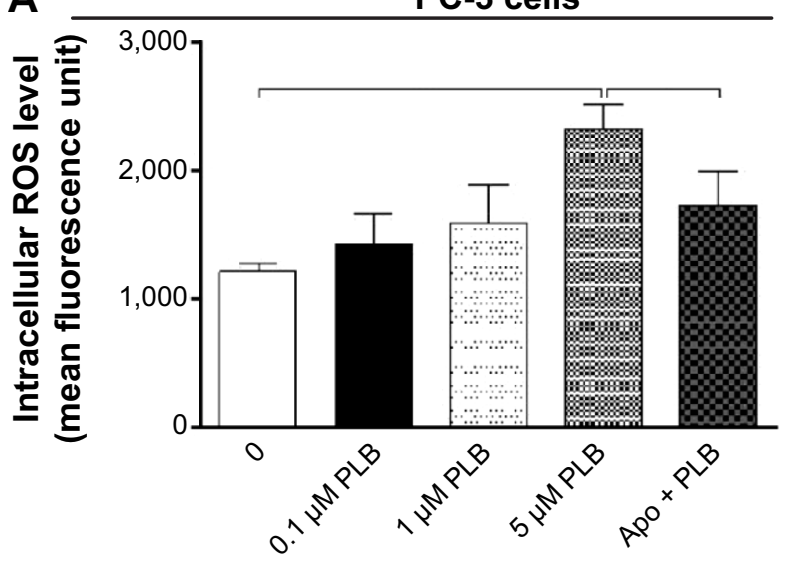

C

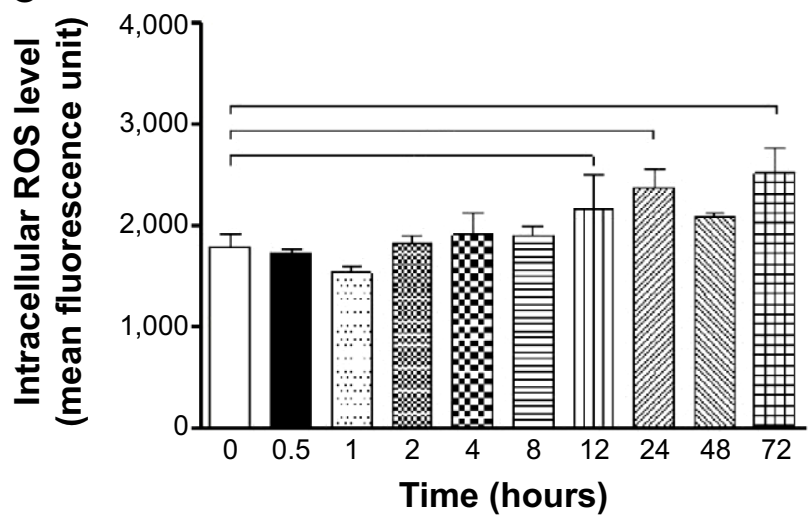

B

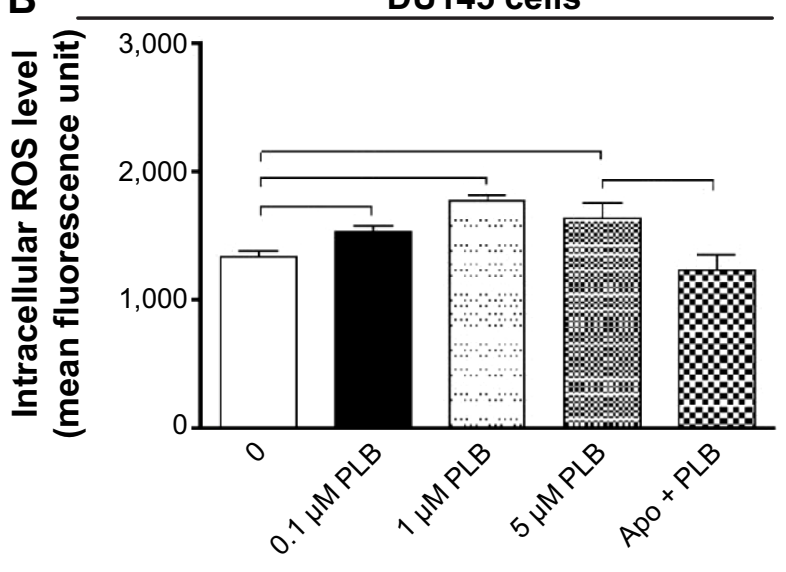

D

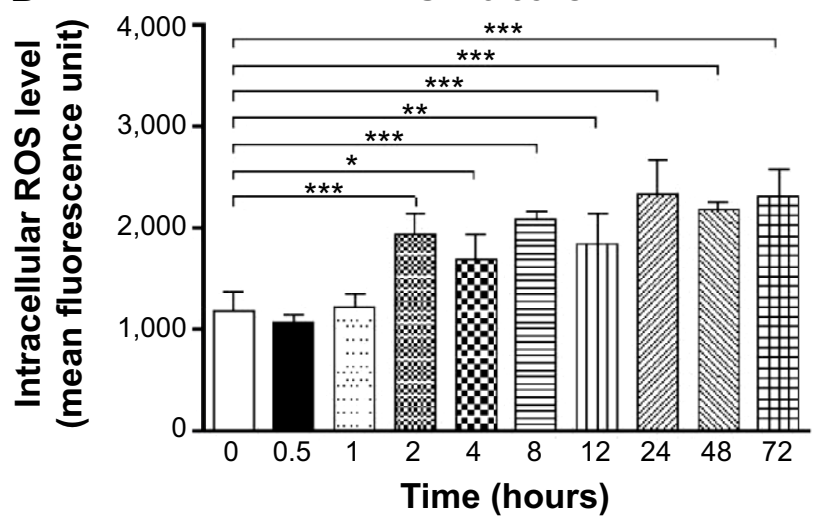

Figure 28 Effect of PLB on the intracellular ROS generation in PC-3 and DUI 45 cells.

Notes: Intracellular ROS level in PC-3 (A) and DUI45 (B) cells treated with PLB at 0.I, I, and $5 \mu$ M for 24 hours; and intracellular ROS level in PC-3 (C) and DUI45 (D) cells treated with $5 \mu$ M PLB over 72 hours. Data are the mean \pm standard deviation of three independent experiments. $* P<0.05$; $* * P<0.0$ I, and $* * * P<0.00$ I by one-way analysis of variance.

Abbreviations: Apo, apocynin; PLB, plumbagin; ROS, reactive oxygen species.

biosynthesis and metabolism; carbohydrate, lipid, steroid, amino acid, and protein metabolism; and signal transduction. In particular, many of the targets predicted based on our bioinformatic tools are associated with cell growth, apoptosis, and related signaling pathways, which have been verified by published data from our group and other groups. $25,28,30,46,70$

To verify the above bioinformatic data and explore whether PC-3 and DU145 cell lines would respond to PLB treatment in similar or different manners, we further analyzed the interactome and related signaling pathways of PLB in PC-3 and DU145 cells using SILAC-based quantitative proteomic approach. The proteomic results revealed that PLB modulated cell cycle regulators, apoptosis- and autophagyrelated signaling pathways, EMT signaling pathways, and redox homeostasis and related signaling pathways, which in turn resulted in an alteration in cell proliferation, cell migration, and cell death with the involvement of a number of function proteins, such as CDK1, CDK2, E-cadherin, PI3K, Akt, mTOR, cytochrome c, caspase 9, caspase 3,
Bcl-2, BAX, p53, PPAR, HSP, Erk1/2, Ras, and Rho. Our proteomic analysis also showed that mTOR signaling pathway was one of the top five signaling pathways regulated by PLB in both PC-3 and DU145 cells and that PLB regulated Nrf2-mediated oxidative response signaling pathway in both cell lines. Importantly, these key proteomic data have been verified by subsequent experiments.

Notably, we observed marked differences in proteomic responses to PLB with regard to the number of related pathways of potential targets between PC-3 and DU145 cells. Our proteomic study showed that PLB altered the expression of a large number of proteins that regulate cell cycle regulators, apoptosis, and EMT signaling pathways in PC-3 cells but not in DU145 cells. This is interesting when both cell lines could be killed by PLB via ROS generation. The reasons for the differential proteomic response are unknown, but may be related to origin of cell lines, remarkably different cytogenetics, and other possible factors. PC3 cells were obtained from a patient with a bone metastasis of grade IV prostate cancer 
and showed a higher metastatic potential compared to DU145 cells, and did not respond to androgens, glucocorticoids, or epidermal or fibroblast growth factors. ${ }^{71}$ The significantly different cytogenetic characteristics of PC-3 and DU145 cells may be another contributing factor. ${ }^{72,73} \mathrm{PC}-3$ cells have a unique karyotype in the absence of chromosomes 2, 3, 5, 15 , and $\mathrm{Y}^{74}$ The centromere 8 copy number was substantially different between PC-3 and DU145 cells. ${ }^{75}$ The copy number of centromere 8 with the highest observed frequency was two (79.4\%) in PC-3 cells and three (70.\%) in DU145 cells. ${ }^{75}$ A recent study indicated that DU145 cells have no detectable autophagy upon treatment with a known autophagic inducer, valproic acid, indicating a defect of autophagy in this cell line. ${ }^{76}$ In addition, the different batches and passages of cells used for separate experiments might also contribute to the different responses to PLB treatment as well.

In the present study, the proteomic data showed differential responses to PLB treatment with regard to cell cycle between PC-3 and DU145 cells. PLB regulated cell cycle at $G_{1}$ and $\mathrm{G}_{2}$ checkpoints involving a number of cell cycle regulators in PC-3 cells, such as RPL11, RPL5, HDAC2, PA2G4, GNL3, SKP1, YWHAQ, PRKDC, YWHAG, YWHAE, YWHAH, YWHAB, YWHAZ, SFN, SKP1, and CDK1, which consequently result in alterations in cell cycle distribution. However, the proteomic analysis did not show a significant modulating effect of cell cycle signaling pathways in DU145 cells. Indeed, we found a differential effect of PLB on cell cycle distribution in PC-3 and DU145 cells using flow cytometry. PLB concentration-dependently arrested PC-3 and DU145 cells in $\mathrm{G}_{2} / \mathrm{M}$ and $\mathrm{G}_{1}$ phase, respectively. We further explored the effect of PLB on the key regulators in cell cycle checkpoints including $\mathrm{CDC} 2$, cyclin $\mathrm{B} 1, \mathrm{CDK} 2$, and cyclin $\mathrm{D}$ in both cell lines. The $\mathrm{CDC} 2-$ cyclin $\mathrm{B} 1$ complex is pivotal in regulating the $\mathrm{G}_{2} / \mathrm{M}$ phase transition and mitosis. We observed a significant decrease in the expression level of cyclin B1 and CDC2 in PC-3 cells treated with PLB, providing an explanation for the effect of PLB on $\mathrm{G}_{2} / \mathrm{M}$ phase arrest in PC-3 cells. We observed that the expression of p53 and $\mathrm{p} 21 \mathrm{Waf} 1 / \mathrm{Cip} 1$ was concentration- and time-dependently increased in PC-3 cells treated with PLB, which probably contributes to the inhibitory effect of PLB on cell proliferation and inducing effect on cell cycle arrest in PC-3 cells. For DU145 cells, a significant reduction in the expression of CDK2 and cyclin D was observed. We also found that PLB exhibited a concentration-dependent inducing effect on the expression of p21 Waf1/Cip1 and p27 Kip1 in DU145 cells. Furthermore, PLB increased the expression of p53 in DU145 cells. The results indicate that upregulation of $\mathrm{p} 53$, p21 Waf1/Cip1, and p27 Kip1 expression, and suppression of CDK2 and cyclin D by PLB may result in the $\mathrm{G}_{1}$ phase arrest in DU145 cells. These results provide further evidence that both PC-3 and DU145 cells differentially respond to PLB treatment and the cells are arrested in distinct phases.

Previous studies demonstrate that apoptosis and autophagy are two predominant cell death routes regulated by PLB in various cancer cells. ${ }^{25,27,28,30,33,46}$ In agreement with previous studies, our proteomic findings confirmed that PLB exhibited remarkable regulatory effects on apoptosis and autophagy in both PC-3 and DU145 cells via modulating the expression or activity of apoptotic and autophagic proteins and signaling pathways, including mTOR, p38 MAPK, and mitochondria-dependent pathways. Intriguingly, the apoptotic signaling pathway was only observed in PC-3 cells in response to PLB treatment. On the other hand, our Western blot assay showed similar apoptosis- and autophagy-inducing effects of PLB in both PC-3 and DU145 cells by regulating the expression of cytochrome c, caspase 9, caspase 3, Bcl-2, and BAX and the phosphorylation of PI3K, mTOR, Akt, and p38MAPK. Although the SILAC-based proteomics did not show a direct alteration in apoptosis in DU145 cells, the mitochondria-related apoptosis may be attributed to multiple modulating effects of PLB on other functional proteins and signaling pathways, such as the p53- and p38MAPKmediated signaling pathways. These data also show that SILAC-based quantitative proteomic analysis is much more sensitive than routine protein quantification assays such as Western blot and enzyme-linked immunosorbent assay in terms of identification of molecular networks and discrimination of various signaling pathways that are involved in the anticancer effects of PLB.

EMT is characterized by epithelial cells that lose their polarization and specialized junction structures, undergoing cytoskeleton reorganization and acquiring morphological and functional features of mesenchymal-like cells..$^{20,21}$ In clinic, the prostate cancer patient mortality is mainly attributed to the spread of cancerous cells to areas outside the prostate gland and the inadequate strategies to effectively block progression to metastasis; EMT plays a critical role in this process. ${ }^{22}$ In primary prostate cancer cells, reduction or loss of expression of E-cadherin and $\beta$-catenin were observed. ${ }^{22}$ In our proteomic study, we observed marked regulatory effects of PLB on the expression of a number of functional proteins that modulate epithelial adherent junction signaling pathway in PC-3 cells only. These modulating effects have been validated by our Western blotting experiments. The validation results showed that PLB significantly increased 
the ratio of E-cadherin over N-cadherin which would result in an EMT inhibition in prostate cancer. Furthermore, PLB increased the expression level of ZO-1 but suppressed the expression of snail, slug, TCF-8, and vimentin in PC-3 cells. Although there was no remarkable alteration in proteomic responses with regard to EMT-related function proteins and signaling pathways in DU145 cells treated with PLB, the validation experiments showed a similar inhibitory effect of PLB on the expression of a number of functional proteins that regulate EMT in DU145 cells. Taken together, our findings suggest that inhibition of EMT progression is one of the beneficial actions of PLB contributing to its anticancer effects in prostate cancer therapy. Again, SILAC-based quantitative proteomic analysis can discriminate the role of EMT modulation in the anticancer effects of PLB on PC-3 and DU145 cells.

Moreover, there is increasing evidence indicating the important role of Sirt1 in the regulation of cancer cell growth, cell death, and metastasis. ${ }^{54,77}$ Sirt1 deacetylates histones, p300, p53, forkhead box class O family members, and NF- $\kappa \mathrm{B}$, which regulate cellular stress response and cell survival. ${ }^{54}$ It also regulates PPAR- $\gamma$, AMPK, and mTOR with regard to cellular energy metabolism and autophagy. ${ }^{54}$ Our proteomic findings showed that the PLB regulated PPAR- $\gamma$, AMPK, p53, and mTOR-associated signaling pathways, which may be attributed to the regulatory effect of PLB on Sirt1 in PC-3 and DU145 cells. Importantly, the proteomic data showed that PLB treatment had a regulated effect on NAMPT in $\mathrm{NAD}^{+}$biosynthesis signaling pathway, which is crucial for functional Sirt1. Consistently, our Western blotting results showed that PLB treatment significantly decreased the expression level of Sirt1 in both cell lines. Of note, it has been reported that silencing Sirt1 can promote the shift to an epithelial morphology in prostate cancer cells. ${ }^{23}$ In agreement with the previous study, we found that inhibition of Sirt1 increased the ratio of E-cadherin over N-cadherin in PC-3 and DU145 cells. The results showed that suppression of Sirt1 prevented EMT progress in prostate cancer cells. Moreover, we observed that inhibition of Sirt1 enhanced the inducing effect of PLB on the ratio of E-cadherin over N-cadherin in PC-3 cells, which indicated that PLB inhibited EMT through a Sirt1-mediated pathway.

Moreover, a number of studies have shown that the ROS-inducing effect of PLB contributes to its cancer cell killing effect in various cancer cell lines. ${ }^{24,30,32-34}$ Our quantitative proteomic analysis uncovered that PLB modulated several critical signaling pathways related to intracellular ROS generation and oxidative stress, including oxidative phosphorylation, Nrf2-mediated oxidative stress response, and superoxide radical degradation with the involvement of a number of enzymes and proteins. We have confirmed that PLB significantly promoted intracellular ROS generation in PC-3 and DU145 cells. Taken together, these results have revealed that the ROS-inducing effect is one of the key events involved in the anticancer effects of PLB.

Our SILAC-based proteomic approach showed significant advantages over the conventional proteomic methods, such as two-dimensional polyacrylamide gel electrophoresis or surface-enhanced laser desorption/ionization mass spectrometry. Although they were primarily used to analyze the protein expression profiles, they cannot quantitatively and easily identify the individual proteins. ${ }^{36,78}$ Compared to single-labeled SILAC proteomic approach, our doublelabeled approach $\left({ }^{13} \mathrm{C}_{6}\right.$-L-lysine and ${ }^{13} \mathrm{C}_{6} /{ }^{15} \mathrm{~N}_{4}$-L-arginine $)$ also showed obvious advantages. For example, Everley et $\mathrm{al}^{79}$ identified 444 proteins from the microsomal fractions of prostate cancer cells including PC3M and PC3M-LN4 cells with varying metastatic potential using ${ }^{13} \mathrm{C}_{6}$-L-lysine SILAC-based proteomic approach. Both of these cell types are derived from PC-3 cells and exhibit low (PC3M) and high (PC3M-LN4) metastatic ability. Of these, 60 were upregulated greater than threefold in the highly metastatic cells, whereas 22 were downregulated by equivalent amounts. We depicted the global proteomic responses to PLB treatment with regard to cell proliferation, cell growth, cell migration, programmed cell death, and ROS production in PC-3 cells via quantification of 1,225 proteins and 341 related signaling pathways, and the double-labeled SILAC-based proteomic approach systematically elicited the network of potential molecular targets and related signaling pathways for PLB in a quantitative manner. Taken together, the double-labeled SILAC-based approach provides a powerful strategy for interactome characterization, new drug target identification, and biomarker determination for diagnosis and treatment of cancer.

Our new findings from the SILAC-based quantitative proteomic analysis have important implications for the subtype classification of prostate-cancer-based protein expression profiles. These SILAC-based data can classify cancer subtypes as well as reveal cancer-specific mechanistic changes. For example, SILAC-based quantitative proteomic assay has been used to classify diffusive large B-cell lymphoma subtypes including activated B-cell-like and germinalcenter B-cell-like subtypes. ${ }^{80,81}$ In one study, SILAC-based proteomic assay yielded a proteome of more than 7,500 identified proteins from mixed cancer cell lines of diffusive 
large B-cell lymphoma. High accuracy of quantification allowed robust separation of subtypes of diffusive large B-cell lymphoma by principal component analysis. The main contributors to the classification included proteins known to be differentially expressed between the subtypes such as the transcription factors IRF4 and SPI1/PU.1, cell surface markers CD44 and CD27, as well as novel candidates. ${ }^{80}$ SILAC-based quantification is a promising new technology for tumor characterization and classification. SILAC-based proteomic assay has not been commonly used for the biomarker identification and classification of prostate cancer. Previous proteomic studies have revealed several biomarkers that can discriminate the subtypes of prostate cancer. ${ }^{82-85}$ For example, lamin A has been found to be a useful discriminatory biomarker for low- and high-grade prostate cancer. ${ }^{85}$ Platelet factor 4, a chemokine with prothrombolytic and antiangiogenic activities, was identified as a stage-specific serologic biomarker for advanced prostate cancer. ${ }^{82}$ In agreement with previous proteomic study, ${ }^{82-85}$ our SILAC-based quantification revealed that $\mathrm{PLB}$ regulated the expression of lamin A and its related apoptotic signaling pathway in PC-3 cells only, which further suggests the potential of SILACbased proteomic approach in biomarker identification and classification of prostate cancer.

Our proteomic data also have implications for personalized cancer treatment. It is well-known that cancer patients respond very differently to chemotherapy and targeted therapies. By incorporating the proteomic data, we can better implement individualized therapies for cancer. A proteomic effort will be necessary to identify useful biomarkers that can classify patient tumor by prognosis and response to therapeutic modalities, and to identify the drivers of tumor behavior that are optimal targets for therapy. An understanding of the effects of targeted therapeutics on signaling networks and homeostatic regulatory loops will be necessary to prevent severe adverse effects as well as to develop rational combinatorial therapies. ${ }^{86,87}$

In summary, we delineated the differences and similarities in the molecular targets and related signaling pathways responding to PLB treatment using SILAC-based proteomic analysis in PC-3 and DU145 cells. The proteomic responses elicited the molecular interactome of PLB in PC-3 and DU145 cells, indicating that the prostate cancer cell killing effect of PLB was mainly ascribed to the regulatory effects on cell cycle, apoptosis, autophagy, EMT, and ROS generation with the involvement of PI3K/Akt/mTOR, p38 MAPK, and Sirt1-mediated signaling pathways. The data have important implications for: better classification of prostate cancer; identification of new therapeutic targets and new biomarkers for the prognosis and response of prostate cancer; and personalized therapy for prostate cancer. However, more studies are needed to elucidate the underlying mechanisms and identify new targets of PLB for prostate cancer therapy.

\section{Acknowledgments}

The authors appreciate the financial support from the Startup Fund of the College of Pharmacy, University of South Florida, Tampa, FL, USA. Dr Zhi-Wei Zhou is a holder of a postdoctoral scholarship from College of Pharmacy, University of South Florida, Tampa, FL, USA.

\section{Disclosure}

The authors report no conflicts of interest in this work.

\section{References}

1. Ahmed HU. Prostate cancer: Time for active surveillance of intermediate-risk disease? Nat Rev Urol. 2013;10(1):6-8.

2. Ferlay J, Soerjomataram I, Ervik M, et al. GLOBOCAN 2012 v1.0, Cancer Incidence and Mortality Worldwide: IARC CancerBase No. 11. [homepage on the Internet]. Lyon, France: International Agency for Research on Cancer; 2013. Available from: http://globocan.iarc.fr, accessed on November 7, 2014.

3. Ferlay J, Shin HR, Bray F, Forman D, Mathers C, Parkin DM. Estimates of worldwide burden of cancer in 2008: GLOBOCAN 2008. Int J Cancer. 2010;127(12):2893-2917.

4. Soerjomataram I, Lortet-Tieulent J, Parkin DM, et al. Global burden of cancer in 2008: a systematic analysis of disability-adjusted life-years in 12 world regions. Lancet. 2012;380(9856):1840-1850.

5. Gunderson K, Wang CY, Wang R. Global prostate cancer incidence and the migration, settlement, and admixture history of the Northern Europeans. Cancer Epidemiol. 2011;35(4):320-327.

6. U.S. Cancer Statistics Working Group. [webpage on the Internet] United States Cancer Statistics: 1999-2010 Incidence and Mortality Web-based Report. Atlanta, GA: 2013. Available from http://apps. nccd.cdc.gov/uscs/. Accessed November 7, 2014.

7. DeSantis CE, Lin CC, Mariotto AB, et al. Cancer treatment and survivorship statistics, 2014. CA Cancer J Clin. 2014;64(4):252-271.

8. Cancer Research UK. [homepage on the Internet]. Cancer statistics report: Cancer incidence and mortality in the UK for the 10 most common cancers December 2013. Available from http://publications. cancerresearchuk.org/cancerstats/statsincidence/reporttop10incmort. html. Accessed November 7, 2014.

9. Klotz L. Nomogram for predicting survival in men with clinically localized prostate cancer who do not undergo definitive therapy. Nat Clin Pract Urol. 2008;5(7):362-363.

10. Albertsen P. Predicting survival for men with clinically localized prostate cancer: what do we need in contemporary practice? Cancer. 2008;112(1):1-3.

11. American Cancer Society. [webpage on the Internet]. Global cancer facts and figures. 2nd edition. Atlanta: 2011Available from: http://www.cancer.org/research/cancerfactsfigures/globalcancerfactsfigures/. Accessed November 7, 2014.

12. Saylor PJ. Prostate cancer: The androgen receptor remains front and centre. Nat Rev Clin Oncol. 2013;10(3):126-128.

13. Wen S, Niu Y, Lee SO, Chang C. Androgen receptor (AR) positive vs negative roles in prostate cancer cell deaths including apoptosis, anoikis, entosis, necrosis and autophagic cell death. Cancer Treat Rev. 2014;40(1):31-40. 
14. Rodrigues DN, Butler LM, Estelles DL, de Bono JS. Molecular pathology and prostate cancer therapeutics: from biology to bedside. J Pathol. 2014;232(2):178-184.

15. Helfand BT, Catalona WJ. The epidemiology and clinical implications of genetic variation in prostate cancer. Urol Clin North Am. 2014; 41(2):277-297.

16. Fang YX, Gao WQ. Roles of microRNAs during prostatic tumorigenesis and tumor progression. Oncogene. 2014;33(2):135-147.

17. Fraser M, Berlin A, Bristow RG, van der Kwast T. Genomic, pathological, and clinical heterogeneity as drivers of personalized medicine in prostate cancer. Urol Oncol. Epub 2014 Apr 22.

18. Crawford ED, Ventii K, Shore ND. New biomarkers in prostate cancer. Oncology (Williston Park). 2014;28(2):135-142.

19. Barve A, Jin W, Cheng K. Prostate cancer relevant antigens and enzymes for targeted drug delivery. J Control Release. 2014;187C:118-132.

20. Zheng H, Kang Y. Multilayer control of the EMT master regulators. Oncogene. 2014;33(14):1755-1763.

21. Lamouille S, Xu J, Derynck R. Molecular mechanisms of epithelialmesenchymal transition. Nat Rev Mol Cell Biol. 2014;15(3):178-196.

22. Nauseef JT, Henry MD. Epithelial-to-mesenchymal transition in prostate cancer: paradigm or puzzle? Nat Rev Urol. 2011;8(8):428-439.

23. Byles V, Zhu L, Lovaas JD, et al. SIRT1 induces EMT by cooperating with EMT transcription factors and enhances prostate cancer cell migration and metastasis. Oncogene. 2012;31(43):4619-4629.

24. Padhye S, Dandawate P, Yusufi M, Ahmad A, Sarkar FH. Perspectives on medicinal properties of plumbagin and its analogs. Med Res Rev. 2012; 32(6):1131-1158.

25. Subramaniya BR, Srinivasan G, Sadullah SS, et al. Apoptosis inducing effect of plumbagin on colonic cancer cells depends on expression of COX-2. PLoS One. 2011;6(4):e18695.

26. Nazeem S, Azmi AS, Hanif S, et al. Plumbagin induces cell death through a copper-redox cycle mechanism in human cancer cells. Mutagenesis. 2009;24(5):413-418.

27. $\mathrm{Xu} \mathrm{KH,} \mathrm{Lu} \mathrm{DP.} \mathrm{Plumbagin} \mathrm{induces} \mathrm{ROS-mediated} \mathrm{apoptosis} \mathrm{in}$ human promyelocytic leukemia cells in vivo. Leuk Res. 2010;34(5): $658-665$.

28. Sun J, McKallip RJ. Plumbagin treatment leads to apoptosis in human K562 leukemia cells through increased ROS and elevated TRAIL receptor expression. Leuk Res. 2011;35(10):1402-1408.

29. Shieh JM, Chiang TA, Chang WT, et al. Plumbagin inhibits TPA-induced MMP-2 and u-PA expressions by reducing binding activities of NF-kappaB and AP-1 via ERK signaling pathway in A549 human lung cancer cells. Mol Cell Biochem. 2010;335(1-2): 181-193.

30. Li YC, He SM, He ZX, et al. Plumbagin induces apoptotic and autophagic cell death through inhibition of the PI3K/Akt/mTOR pathway in human non-small cell lung cancer cells. Cancer Lett. 2014; 344(2):239-259.

31. Sinha S, Pal K, Elkhanany A, et al. Plumbagin inhibits tumorigenesis and angiogenesis of ovarian cancer cells in vivo. Int J Cancer. 2013;132(5):1201-1212.

32. Aziz MH, Dreckschmidt NE, Verma AK. Plumbagin, a medicinal plant-derived naphthoquinone, is a novel inhibitor of the growth and invasion of hormone-refractory prostate cancer. Cancer Res. 2008; 68(21):9024-9032.

33. Powolny AA, Singh SV. Plumbagin-induced apoptosis in human prostate cancer cells is associated with modulation of cellular redox status and generation of reactive oxygen species. Pharm Res. 2008;25(9):2171-2180.

34. Abedinpour P, Baron VT, Chrastina A, Welsh J, Borgstrom P. The combination of plumbagin with androgen withdrawal causes profound regression of prostate tumors in vivo. Prostate. 2013;73(5): 489-499.

35. Ong SE, Mann M. Stable isotope labeling by amino acids in cell culture for quantitative proteomics. Methods Mol Biol. 2007;359:37-52.

36. Mann M. Functional and quantitative proteomics using SILAC. Nat Rev Mol Cell Biol. 2006;7(12):952-958.
37. Ong SE. The expanding field of SILAC. Anal Bioanal Chem. 2012; 404(4):967-976.

38. Wang R, Fang X, Lu Y, Wang S. The PDBbind database: collection of binding affinities for protein-ligand complexes with known threedimensional structures. J Med Chem. 2004;47(12):2977-2980.

39. Yang L, Luo H, Chen J, Xing Q, He L. SePreSA: a server for the prediction of populations susceptible to serious adverse drug reactions implementing the methodology of a chemical-protein interactome. Nucleic Acids Res. 2009;37(Web Server issue):W406-W412.

40. Yang L, Chen J, Shi L, Hudock MP, Wang K, He L. Identifying unexpected therapeutic targets via chemical-protein interactome. PLoS One. 2010;5(3):e9568.

41. Luo H, Chen J, Shi L, et al. DRAR-CPI: a server for identifying drug repositioning potential and adverse drug reactions via the chemicalprotein interactome. Nucleic Acids Res. 2011;39(Web Server issue): W492-W498

42. Huang da W, Sherman BT, Tan Q, et al. The DAVID Gene Functional Classification Tool: a novel biological module-centric algorithm to functionally analyze large gene lists. Genome Biol. 2007; 8(9):R183.

43. Ong SE, Mann M. A practical recipe for stable isotope labeling by amino acids in cell culture (SILAC). Nat Protoc. 2006;1(6): $2650-2660$

44. Lecoeur H. Nuclear apoptosis detection by flow cytometry: influence of endogenous endonucleases. Exp Cell Res. 2002;277(1):1-14.

45. Sunil C, Duraipandiyan V, Agastian P, Ignacimuthu S. Antidiabetic effect of plumbagin isolated from Plumbago zeylanica L. root and its effect on GLUT4 translocation in streptozotocin-induced diabetic rats. Food Chem Toxicol. 2012;50(12):4356-4363.

46. Wang CC, Chiang YM, Sung SC, Hsu YL, Chang JK, Kuo PL. Plumbagin induces cell cycle arrest and apoptosis through reactive oxygen species/c-Jun N-terminal kinase pathways in human melanoma A375.S2 cells. Cancer Lett. 2008;259(1):82-98.

47. Shanware NP, Bray K, Abraham RT. The PI3K, metabolic, and autophagy networks: interactive partners in cellular health and disease. Annu Rev Pharmacol Toxicol. 2013;53:89-106.

48. Morgensztern D, McLeod HL. PI3K/Akt/mTOR pathway as a target for cancer therapy. Anticancer Drugs. 2005;16(8):797-803.

49. Wu WK, Coffelt SB, Cho CH, et al. The autophagic paradox in cancer therapy. Oncogene. 2012;31(8):939-953.

50. Ferreira CG, Epping M, Kruyt FA, Giaccone G. Apoptosis: target of cancer therapy. Clin Cancer Res. 2002;8(7):2024-2034.

51. Fulda S, Galluzzi L, Kroemer G. Targeting mitochondria for cancer therapy. Nat Rev Drug Discov. 2010;9(6):447-464.

52. Laplante M, Sabatini DM. mTOR signaling in growth control and disease. Cell. 2012;149(2):274-293.

53. Dancey J. mTOR signaling and drug development in cancer. Nat Rev Clin Oncol. 2010;7(4):209-219.

54. Preyat N, Leo O. Sirtuin deacylases: a molecular link between metabolism and immunity. J Leukoc Biol. 2013;93(5):669-680.

55. Montecucco F, Cea M, Bauer I, et al. Nicotinamide phosphoribosyltransferase (NAMPT) inhibitors as therapeutics: rationales, controversies, clinical experience. Curr Drug Targets. 2013;14(6):637-643.

56. Keum YS, Choi BY. Molecular and chemical regulation of the Keap1Nrf2 signaling pathway. Molecules. 2014;19(7):10074-10089.

57. Ma Q. Role of nrf2 in oxidative stress and toxicity. Annu Rev Pharmacol Toxicol. 2013;53:401-426.

58. Hu X, Moscinski LC. Cdc2: a monopotent or pluripotent CDK? Cell Prolif. 2011;44(3):205-211.

59. Warfel NA, El-Deiry WS. p21WAF1 and tumourigenesis: 20 years after. Curr Opin Oncol. 2013;25(1):52-58.

60. Yoon MK, Mitrea DM, Ou L, Kriwacki RW. Cell cycle regulation by the intrinsically disordered proteins p21 and p27. Biochem Soc Trans. 2012;40(5):981-988.

61. Carvajal LA, Manfredi JJ. Another fork in the road - life or death decisions by the tumour suppressor p53. EMBO Rep. 2013;14(5): 414-421. 
62. Settembre C, Fraldi A, Medina DL, Ballabio A. Signals from the lysosome: a control centre for cellular clearance and energy metabolism. Nat Rev Mol Cell Biol. 2013;14(5):283-296.

63. Maes H, Rubio N, Garg AD, Agostinis P. Autophagy: shaping the tumor microenvironment and therapeutic response. Trends Mol Med. 2013;19(7):428-446

64. Cannito S, Novo E, di Bonzo LV, Busletta C, Colombatto S, Parola M. Epithelial-mesenchymal transition: from molecular mechanisms, redox regulation to implications in human health and disease. Antioxid Redox Signal. 2010;12(12):1383-1430.

65. Grozinger CM, Chao ED, Blackwell HE, Moazed D, Schreiber SL. Identification of a class of small molecule inhibitors of the sirtuin family of NAD-dependent deacetylases by phenotypic screening. J Biol Chem. 2001;276(42):38837-38843.

66. Bellot GL, Liu D, Pervaiz S. ROS, autophagy, mitochondria and cancer: Ras, the hidden master? Mitochondrion. 2013;13(3):155-162.

67. Dodson M, Darley-Usmar V, Zhang J. Cellular metabolic and autophagic pathways: traffic control by redox signaling. Free Radic Biol Med. 2013;63:207-221.

68. Kaminskyy VO, Zhivotovsky B. Free radicals in cross talk between autophagy and apoptosis. Antioxid Redox Signal. 2014;21(1): 86-102.

69. Hsu YL, Cho CY, Kuo PL, Huang YT, Lin CC. Plumbagin (5-hydroxy2-methyl-1,4-naphthoquinone) induces apoptosis and cell cycle arrest in A549 cells through p53 accumulation via c-Jun NH2-terminal kinasemediated phosphorylation at serine 15 in vitro and in vivo. JPharmacol Exp Ther. 2006;318(2):484-494

70. Yang SJ, Chang SC, Wen HC, Chen CY, Liao JF, Chang CH. Plumbagin activates ERK1/2 and Akt via superoxide, Src and PI3-kinase in 3T3-L1 cells. Eur J Pharmacol. 2010;638(1-3):21-28.

71. Kaighn ME, Narayan KS, Ohnuki Y, Lechner JF, Jones LW. Establishment and characterization of a human prostatic carcinoma cell line (PC-3). Invest Urol. 1979;17(1):16-23.

72. Chen TR. Chromosome identity of human prostate cancer cell lines, PC-3 and PPC-1. Cytogenet Cell Genet. 1993;62(2-3):183-184.

73. Nupponen NN, Hyytinen ER, Kallioniemi AH, Visakorpi T. Genetic alterations in prostate cancer cell lines detected by comparative genomic hybridization. Cancer Genet Cytogenet. 1998;101(1):53-57.

74. Ohnuki Y, Marnell MM, Babcock MS, Lechner JF, Kaighn ME. Chromosomal analysis of human prostatic adenocarcinoma cell lines. Cancer Res. 1980;40(3):524-534.
75. Beheshti B, Park PC, Sweet JM, Trachtenberg J, Jewett MA, Squire JA. Evidence of chromosomal instability in prostate cancer determined by spectral karyotyping (SKY) and interphase fish analysis. Neoplasia. 2001;3(1):62-69.

76. Ouyang DY, Xu LH, He XH, et al. Autophagy is differentially induced in prostate cancer LNCaP, DU145 and PC-3 cells via distinct splicing profiles of ATG5. Autophagy. 2013;9(1):20-32.

77. Feldman JL, Dittenhafer-Reed KE, Denu JM. Sirtuin catalysis and regulation. J Biol Chem. 2012;287(51):42419-42427.

78. Wulfkuhle JD, Liotta LA, Petricoin EF. Proteomic applications for the early detection of cancer. Nat Rev Cancer. 2003;3(4):267-275.

79. Everley PA, Krijgsveld J, Zetter BR, Gygi SP. Quantitative cancer proteomics: stable isotope labeling with amino acids in cell culture (SILAC) as a tool for prostate cancer research. Mol Cell Proteomics. 2004;3(7):729-735.

80. Deeb SJ, D’Souza RC, Cox J, Schmidt-Supprian M, Mann M. SuperSILAC allows classification of diffuse large B-cell lymphoma subtypes by their protein expression profiles. Mol Cell Proteomics. 2012; 11(5):77-89.

81. Deeb SJ, Cox J, Schmidt-Supprian M, Mann M. N-linked glycosylation enrichment for in-depth cell surface proteomics of diffuse large B-cell lymphoma subtypes. Mol Cell Proteomics. 2014;13(1):240-251.

82. Lam YW, Mobley JA, Evans JE, Carmody JF, Ho SM. Mass profiling-directed isolation and identification of a stage-specific serologic protein biomarker of advanced prostate cancer. Proteomics. 2005;5(11):2927-2938.

83. Sardana G, Marshall J, Diamandis EP. Discovery of candidate tumor markers for prostate cancer via proteomic analysis of cell cultureconditioned medium. Clin Chem. 2007;53(3):429-437.

84. Al-Ruwaili JA, Larkin SE, Zeidan BA, et al. Discovery of serum protein biomarkers for prostate cancer progression by proteomic analysis. Cancer Genomics Proteomics. 2010;7(2):93-103.

85. Skvortsov S, Schäfer G, Stasyk T, et al. Proteomics profiling of microdissected low- and high-grade prostate tumors identifies Lamin A as a discriminatory biomarker. J Proteome Res. 2011;10(1):259-268.

86. Gonzalez-Angulo AM, Hennessy BT, Mills GB. Future of personalized medicine in oncology: a systems biology approach. J Clin Oncol. 2010;28(16):2777-2783.

87. Tian Q, Price ND, Hood L. Systems cancer medicine: towards realization of predictive, preventive, personalized and participatory (P4) medicine. J Intern Med. 2012;271(2):111-121.

\section{Publish your work in this journal}

Drug Design, Development and Therapy is an international, peerreviewed open-access journal that spans the spectrum of drug design and development through to clinical applications. Clinical outcomes, patient safety, and programs for the development and effective, safe, and sustained use of medicines are a feature of the journal, which

\section{Dovepress}

has also been accepted for indexing on PubMed Central. The manuscript management system is completely online and includes a very quick and fair peer-review system, which is all easy to use. Visit http://www.dovepress.com/testimonials.php to read real quotes from published authors. 